\title{
MODULAR INVARIANCE OF CHARACTERS OF VERTEX OPERATOR ALGEBRAS
}

\author{
YONGCHANG ZHU
}

\section{INTRODUCTION}

In contrast with the finite dimensional case, one of the distinguished features in the theory of infinite dimensional Lie algebras is the modular invariance of the characters of certain representations. It is known $[\mathrm{Fr}],[\mathrm{KP}]$ that for a given affine Lie algebra, the linear space spanned by the characters of the integrable highest weight modules with a fixed level is invariant under the usual action of the modular group $S L_{2}(\mathbf{Z})$. The similar result for the minimal series of the Virasoro algebra is observed in [Ca] and [IZ]. In both cases one uses the explicit character formulas to prove the modular invariance. The character formula for the affine Lie algebra is computed in $[\mathrm{K}]$, and the character formula for the Virasoro algebra is essentially contained in $[\mathrm{FF}]$; see $[\mathrm{R}]$ for an explicit computation.

This mysterious connection between the infinite dimensional Lie algebras and the modular group can be explained by the two dimensional conformal field theory. The highest weight modules of affine Lie algebras and the Virasoro algebra give rise to conformal field theories. In particular, the conformal field theories associated to the integrable highest modules and minimal series are rational. The characters of these modules are understood to be the holomorphic parts of the partition functions on the torus for the corresponding conformal field theories. From this point of view, the role of the modular group $S L_{2}(\mathbf{Z})$ is manifest.

In the study of conformal field theory, physicists arrived at the notion of chiral algebras (see e.g. [MS]). Independently, in the attempt to realize the Monster sporadic group as a symmetry group of certain algebraic structure, an infinite dimensional graded representation of the Monster sporadic group, the so called Moonshine module, was constructed in [FLM1]. This algebraic structure was later found in $[\mathrm{Bo}]$ and called the vertex algebra; the first axioms of vertex operator algebras were formulated in that paper. The proof that the Moonshine module is a vertex operator algebra and the Monster group acts as its automorphism group was given in [FLM2]. Notably the character of the Moonshine module is also a modular function, namely $j(\tau)-744$. It turns out that the vertex operator algebra can be regarded as a rigorous mathematical definition of the chiral algebras in the physical literature. And it is expected that a pair of isomorphic vertex operator algebras and their representations (corresponding to the holomorphic and antiholomorphic sectors) are the basic objects needed to build a conformal field theory of a certain type.

Received by the editors January 24, 1994 and, in revised form, January 31, 1995.

1991 Mathematics Subject Classification. Primary 17B65. 
According to the physical arguments described in [MS], in order to construct a conformal field theory based on representations of chiral algebras, some consistency conditions are needed. A key condition among them is the modular invariance of the characters of the sectors (irreducible representations). The purpose of this paper is to prove that the modular invariance is a consequence of the axioms of vertex operator algebras together with some finiteness conditions. Starting from the basic axioms, we formulate the notions of the correlation functions and the conformal block for the torus, and prove the modular invariance for all the correlation functions. Since the characters are the simplest correlation functions on the torus, our main theorem (Theorem 5.3.2) in particular gives a uniform proof of the modular invariance of the characters of integral highest weight representations of affine Lie algebras, minimal series of the Viraroso algebra, the Moonshine module of the Monster group, and the lattice models.

To explain our approach in more details let us give informally some concepts about vertex operator algebras (see Section 1 for details). In contrast to the usual algebraic structures (e.g., associative algebras, Lie algebras) where there is one operation, for a vertex operator algebra $V$, however, there are infinitely many bilinear operations parametrized by integers: $a *_{n} b(a, b \in V, n \in \mathbf{Z})$. For $a \in V$, we define a linear operator $a(n) \in \operatorname{End}(V)$ associated to " $n$-th multiplication" by $a$ by $a(n) b=a *_{n} b$, and form a generating function $Y(a, z)=\sum_{n=-\infty}^{\infty} a(n) z^{-n-1}$, which is called the vertex operator of $a$. It is more natural to study vertex operators $Y(a, z)$ as a whole than to study operators $a(n)$ 's separately, because $Y(a, z)$ has physical meaning as the expansion of a meromorphic quantum field at a point with local coordinate $z$ of a Riemann surface. As we shall see later, it is also technically more accessible to study $Y(a, z)$. The main axiom satisfied by the vertex operators is the Jacobi identity, which is a combination of the Jacobi identity for Lie algebras and the Cauchy formula for contour integrals. The Jacobi identity is now understood as a rigorous formulation of "operator product expansion" of conformal field theory in physical literature (to be more precise, it axiomizes the notion of operator product expansions and their commutativity and associativity for fields in the 0 sectors). The other main axioms are the existence of two special elements $1 \in V$, called the identity element, and $\omega \in V$, called the Virosoro element, such that $Y(1, z)=1$ and the operator components $L_{n}$ in $Y(\omega, z)=\sum_{n=-\infty}^{\infty} L_{n} z^{-n-2}$ form a copy of the Virasoro algebra. Concepts such as representations, subpresentations, irreducible representations are defined similarly as for the usual algebras.

An irreducible representation $M$ has the form $M=\bigoplus_{n=0}^{\infty} M_{n}$, where every $M_{n}$ has finite dimension and $L_{0}$ acts on $M_{n}$ as a scalar $n+h$ for some constant $h$. The character of $M$ is defined to be the formal power series

$$
C h(M)=\left.t r\right|_{M} q^{L_{0}-\frac{c}{24}}=\sum_{n=0}^{\infty} \operatorname{dim}\left(M_{n}\right) q^{n-\frac{c}{24}},
$$

where $c$ is the central charge of the Virasoro algebra. One of our main theorems (Theorem 5.3.3) is that if a vertex operator algebra $V$ satisfies some finiteness condition (given in Section 4.4), and $V$ has finitely many equivalence classes of irreducible representations $M_{1}, \ldots, M_{m}$, and every representation is a direct sum of irreducible ones, then $C h\left(M_{1}\right), \ldots, C h\left(M_{m}\right)$ converge absolutely on the complex domain $|q|<1$ and the linear space spanned by $C h\left(M_{i}\right)$ 's are invariant under the 
action of $S L_{2}(\mathbf{Z})$ (where we consider $C h\left(M_{i}\right)$ as a holomorphic function on the upper half plane by the transformation $\left.q=e^{2 \pi i \tau}\right)$.

The proof consists of three main steps. The main idea is to consider $C h\left(M_{i}\right)$ 's as correlation functions for the identity element 1 on the torus. In Step 1, we define abstractly the correlation function and conformal block on the torus for a given vertex operator algebra (Definition 4.1.1, Definition 4.1.2), and prove that $S L_{2}(\mathbf{Z})$ acts on the conformal block (Theorem 5.1.1). In Step 2 (Sections 4.2-4.4), we prove that every irreducible representation of the vertex operator algebra gives rise to a system of correlation functions on the torus by taking $q$-trace of vertex operators and therefore gives a vector in the conformal block (Theorem 4.4.3). In Step 3 (Sections 5.1-5.3), we prove that every system of correlation functions on the torus is given by irreducible representations (Theorem 5.2.1). Then the theorem described above is a special case of the modular invariance of general correlation functions constructed as the $q$-trace of a product of vertex operators (Theorem 5.3 .2 ).

We also construct a functor $V \mapsto A(V)$ assigning to a vertex operator algebra an associative algebra (Theorem 2.1.1). And we prove that the equivalence classes of the irreducible representations of $V$ are in one-to-one correspondence with to equivalence classes of the irreducible representations of $A(V)$ (see Theorems 2.1.2, 2.2.1 and 2.2.2). $A(V)$ has much simpler structure than $V$, for example, the one-to-one correspondence theorem implies that if $V$ is rational then $A(V)$ is semisimple. The associative algebra $A(V)$ also plays a crucial role in the proof of the modular invariance (see the proof of Theorem 5.3.1). It seems that $A(V)$ controls the behavior of the correlation functions at a double point of a degenerate Riemann surface.

We include some expository materials to make this paper self-contained. Section 1 reviews basic definitions of vertex operator algebras and gives proofs of results needed later. Section 3 recalls some formulas about Weierstrass $\wp$-functions and Eisentein series.

Throughout this paper, we use the convention that the contour integral $\int_{C} f(z) d z$ is normalized so that $\int_{C} \frac{1}{z} d z=1$ for a contour $C$ surrounding 0 , and we will denote by $\mathbf{C}, \mathbf{Z}, \mathbf{N}$ the set of complex numbers, the set of integers and the set of nonnegative integers respectively.

\section{Vertex operator algebras}

In this section we recall some basic definitions on vertex operator algebras and give proofs of some basic facts needed later. All the results are either elementary or in [FLM2] and [FHL].

1.1. Formal calculus. We discuss formal power series and fix the necessary notations. For a vector space $V$, we define a vector space

$$
V\left[\left[z, z^{-1}\right]\right]=\left\{\sum_{n \in \mathbf{Z}} v_{n} z^{n} \mid v_{n} \in V\right\}
$$

and a subspace of $V\left[\left[z, z^{-1}\right]\right]$ :

$$
V((z))=\left\{\sum_{n \in \mathbf{Z}} v_{n} z^{n} \mid v_{n} \in V, v_{n}=0 \text { for sufficiently small } n\right\},
$$


where $z$ is a formal variable. We will also use analogous notations for several variables.

For a rational function $f(z, w)$ with possible poles only at $z=w, z=0$ and $w=0$, we denote by $\iota_{z, w} f(z, w), \iota_{w, z} f(z, w)$ and $\iota_{w, z-w} f(z, w)$ the power series expansions of $f(z, w)$ on the domains $|z|>|w|>0,|w|>|z|>0$ and $|w|>$ $|z-w|>0$ respectively; they are elements in $\mathbf{C}\left[\left[z, z^{-1} ; w, w^{-1}\right]\right], \mathbf{C}\left[\left[z, z^{-1} ; w, w^{-1}\right]\right]$ and $\mathbf{C}\left[\left[w, w^{-1} ; z-w,(z-w)^{-1}\right]\right]$ respectively (here $w, z-w$ in $\mathbf{C}\left[\left[w, w^{-1} ; z-w\right.\right.$, $\left.\left.(z-w)^{-1}\right]\right]$ are regarded as independent variables). For example,

$$
\begin{aligned}
& \iota_{z, w}\left(z^{m} w^{n}(z-w)^{l}\right)=\sum_{i=0}^{\infty}(-1)^{i}\left(\begin{array}{l}
l \\
i
\end{array}\right) z^{m+l-i} w^{n+i}, \\
& \iota_{w, z}\left(z^{m} w^{n}(z-w)^{l}\right)=\sum_{i=0}^{\infty}(-1)^{l+i}\left(\begin{array}{l}
l \\
i
\end{array}\right) z^{m+i} w^{n+l-i}, \\
& \iota_{w, z-w}\left(z^{m} w^{n}(z-w)^{l}\right)=\sum_{i=0}^{\infty}\left(\begin{array}{c}
m \\
i
\end{array}\right) w^{m+n-i}(z-w)^{l+i} .
\end{aligned}
$$

We will use the same notations in the following more general situation. If $f(z, w)$ is a product of a rational function with possible poles only at $z=w, z=0$ and $w=0$ and a holomorphic function defined on a neighborhood of the origin of $\mathbf{C}^{2}$, it still makes sense to speak of the power series expansions of $f(z, w)$ in the domains $|z|>|w|>0,|w|>|z|>0$ and $|w|>|z-w|>0$. So the notations $\iota_{z, w} f(z, w)$, $\iota_{w, z} f(z, w)$ and $\iota_{w, z-w} f(z, w)$ make sense.

We define the formal residue as follows: for $f(z) \in V\left[\left[z, z^{-1}\right]\right]$,

$$
\text { Res } f(z)=\text { coefficient of } z^{-1} \text { in } f(z) \text {. }
$$

In the several variable case, we use a subindex on Res to indicate the variable with respect to which the formal residue is to be taken. For example, for $f(z, w) \in$ $V\left[\left[z, z^{-1}, w, w^{-1}\right]\right]$, we have $\operatorname{Res}_{z} f(z, w) \in V\left[\left[w, w^{-1}\right]\right]$.

Formal residue enjoys some properties of contour integration. We have

(1) "Integration by parts". For $f(z) \in \mathbf{C}((z))$ and $g(z) \in V((z))$,

$$
\operatorname{Res}\left(f(z) \frac{d}{d z} g(z)\right)=-\operatorname{Res}\left(\frac{d}{d z} f(z) g(z)\right)
$$

where $f(z) \in \mathbf{C}((z))$ and $g(z) \in V((z))$.

(2) Formula for change of variable. For $g(w)=\sum_{m \geq M} v_{m} w^{m} \in V((w))$ and $f(z)=\sum_{n=1}^{\infty} a_{n} z^{n} \in \mathbf{C}[[z]]$ with $a_{1} \neq 0$, the power series $g(f(z)) \in V((z))$ is defined as

$$
g(f(z))=\sum_{m \geq M} v_{m} f(z)^{m}=\sum_{m \geq M} \sum_{j=0}^{\infty} v_{m}\left(a_{1} z\right)^{m}\left(\begin{array}{c}
m \\
j
\end{array}\right) \bar{f}^{j}
$$

where $\bar{f}=\sum_{i=2}^{\infty} \frac{a_{i}}{a_{1}} z^{i-1}$. We have the following formula for this change of variable:

$$
\operatorname{Res}_{w} g(w)=\operatorname{Res}_{z}\left(g(f(z)) \frac{d}{d z} f(z)\right)
$$


(3) Cauchy Theorem. If $f(z, w)$ is a multiplication of a rational function with possible poles at $z=w, z=0$ and $w=0$ and a holomorphic function defined on a neighborhood of the origin of $\mathbf{C}^{2}$, then

$$
\operatorname{Res}_{z-w}\left(\iota_{w, z-w} f(z, w)\right)=\operatorname{Res}_{z}\left(\iota_{z, w} f(z, w)\right)-\operatorname{Res}_{z}\left(\iota_{w, z} f(z, w)\right) .
$$

Note that both sides of (1.1.4) are in $\mathbf{C}\left[\left[w, w^{-1}\right]\right]$.

Formulas (1.1.2)-(1.1.4) and their obvious extensions will be used in the computations involving vertex operators.

\subsection{Definitions of vertex operator algebras and modules.}

Definition 1.2.1. A vertex operator algebra is a graded vector space $V=\bigoplus_{n=0}^{\infty} V_{n}$ equipped with a linear map

$$
\begin{aligned}
V & \rightarrow(\text { End } V)\left[\left[z, z^{-1}\right]\right], \\
a & \mapsto Y(a, z)=\sum_{n \in \mathbf{Z}} a(n) z^{-n-1} \quad(a(n) \in \text { End } V)
\end{aligned}
$$

(we call $Y(a, z)$ the vertex operator of $a$ ) and with two distinguished vectors $1 \in V_{0}$ (called the identity element), $\omega \in V_{2}$ (called the Virasoro element) satisfying the following conditions for $a, b \in V$ :

$$
\begin{aligned}
& a(n) b=0 \quad \text { for } n \text { sufficiently large } \\
& Y(1, z)=1 ; \\
& Y(a, z) 1 \in V[[z]] \text { and } \lim _{z \rightarrow 0} Y(a, z) 1=a ;
\end{aligned}
$$

the vertex operator $Y(\omega, z)=\sum_{n \in \mathbf{Z}} L_{n} z^{-n-2}$ generates a copy of the Virasoro algebra:

$$
\left[L_{m}, L_{n}\right]=(m-n) L_{m+n}+\delta_{m+n, 0} \frac{m^{3}-m}{12} c,
$$

where $c$ is a constant called the rank of $V$; and

$$
\begin{aligned}
& L_{0} a=n a=\operatorname{deg} a a \quad \text { for } \quad a \in V_{n}, \\
& Y\left(L_{-1} a, z\right)=\frac{d}{d z} Y(a, z) ;
\end{aligned}
$$

and the following Jacobi identity holds for every $m, n, l \in \mathbf{Z}$ :

$$
\begin{gathered}
\operatorname{Res}_{z-w}\left(Y(Y(a, z-w) b, w) \iota_{w, z-w} F(z, w)\right) \\
=\operatorname{Res}_{z}\left(Y(a, z) Y(b, w) \iota_{z, w} F(z, w)\right)-\operatorname{Res}_{z}\left(Y(b, w) Y(a, z) \iota_{w, z} F(z, w)\right),
\end{gathered}
$$

where $F(z, w)=z^{m} w^{n}(z-w)^{l}$. This completes the definition.

Identity (1.2.7) is equivalent to

$$
\sum_{i=0}^{\infty}\left(\begin{array}{c}
m \\
i
\end{array}\right) Y(a(l+i) b, w) w^{m+n-i}
$$

$$
=\sum_{i=0}^{\infty}(-1)^{i}\left(\begin{array}{l}
l \\
i
\end{array}\right) a(m+l-i) Y(b, w) w^{n+i}-\sum_{i=0}^{\infty}(-1)^{l+i}\left(\begin{array}{l}
l \\
i
\end{array}\right) Y(b, w) a(m+i) w^{n+l-i} .
$$


Taking the coefficient of $w^{-1}$ in both sides of (1.2.8), we have

$$
\begin{gathered}
\sum_{i=0}^{\infty}\left(\begin{array}{c}
m \\
i
\end{array}\right)(a(l+i) b)(m+n-i) \\
=\sum_{i=0}^{\infty}(-1)^{i}\left(\begin{array}{l}
l \\
i
\end{array}\right) a(m+l-i) b(n+i)-\sum_{i=0}^{\infty}(-1)^{l+i}\left(\begin{array}{l}
l \\
i
\end{array}\right) b(n+l-i) a(m+i) .
\end{gathered}
$$

By (1.2.1), for a fixed $a, b, v \in V$, finitely many vectors among $a(l+i) b, b(n+i) v$ and $a(m+i) v(i \geq 0)$ are non-zero, so all three terms in (1.2.9) are well-defined linear operators on $V$. The Jacobi identity is equivalent to (1.2.9) for every $m, n, l \in \mathbf{Z}$.

We give some immediate consequences of the definition. We have relations:

$$
\begin{aligned}
& Y(a, z)=0 \quad \text { iff } \quad a=0, \\
& {[a(m), Y(b, w)]=\sum_{i=0}^{\infty}\left(\begin{array}{c}
m \\
i
\end{array}\right) Y(a(i) b, w) w^{m-i},} \\
& {\left[L_{-1}, Y(a, z)\right]=\frac{d}{d z} Y(a, z),} \\
& {\left[L_{0}, Y(a, z)\right]=\frac{d}{d z} Y(a, z) z+Y\left(L_{0} a, z\right),} \\
& a(n) V_{m} \subset V_{m+\operatorname{deg} a-n-1} \text { for } a \text { homogeneous, } \\
& a(n) 1=0 \text { for } n \geq 0, \\
& Y(a, z) 1=\exp \left(z L_{-1}\right) a, \\
& a(-k-1) 1=\frac{1}{k !}\left(L_{-1}\right)^{k} a \text { for } k \geq 0 .
\end{aligned}
$$

We sketch the proofs of the above relations. (1.2.10) follows directly from (1.2.3). (1.2.11) is obtained by specifying $l=0, n=0$ in (1.2.8). (1.2.12) and (1.2.13) are proved by using (1.2.11), (1.2.5) and (1.2.6). (1.2.14) follows from (1.2.13) directly. (1.2.15) follows from (1.2.3). (1.2.16) and (1.2.17) are equivalent. To prove (1.2.17), using (1.2.12), we have $\left(a d L_{-1}\right)^{k} Y(a, z)=\left(\frac{d}{d z}\right)^{k} Y(a, z)$. Applying this identity to 1 and taking constant terms, then using $L_{-1} 1=\omega(0) 1=0$, we obtain $\left(L_{-1}\right)^{k} a=k ! a(-k-1)$.

From (1.2.11) we see that the operators $a(n)(a \in V, n \in \mathbf{Z})$ are closed under the Lie bracket. And from (1.2.14), we see that for a homogeneous element $a$, the operator $a(n)(n \in \mathbf{Z})$ maps a homogeneous subspace $V_{m}$ into the homogeneous subspace $V_{m+\operatorname{deg} a-1-n}$, so we write $\operatorname{deg}(a(n))=\operatorname{deg} a-1-n$. For $k>0$, the operator $a(\operatorname{deg} a-1+k)$ lowers the degree; $a(\operatorname{deg} a-1-k)$ raises the degree, and $a(\operatorname{deg} a-1)$ preserves the degree.

Definition 1.2.2. A strong representation for $V$ or a strong module for $V$ is a C-graded vector space $M=\bigoplus_{s \in \mathbf{C}} M_{s}$ such that $M_{s}=0$ for $s$ sufficiently small in the sense of modification by an integer, i.e., for fixed $s \in \mathbf{C}, M_{s-n}=0$ for sufficiently large integers $n$, equipped with a linear map

$$
\begin{aligned}
& V \rightarrow(\text { End } M)\left[\left[z, z^{-1}\right]\right], \\
& a \mapsto Y_{M}(a, z)=\sum_{n \in \mathbf{Z}} a(n) z^{-n-1}
\end{aligned}
$$


such that "all the defining properties of a vertex operator algebra that make sense hold." That is, for $a, b \in V$, and $v \in M$ :

$$
a(n) v=0 \text { for } n \text { sufficiently large; }
$$

$$
=\operatorname{Res}_{z}\left(Y_{M}(a, z) Y_{M}(b, w) \iota_{z, w} F(z, w)\right)-\operatorname{Res}_{z}\left(Y_{M}(b, w) Y_{M}(a, z) \iota_{w, z} F(z, w)\right),
$$

for every rational function $F(z, w)=z^{m} w^{n}(z-w)^{l}(m, n, l \in \mathbf{Z})$; the Virasoro relations hold on $W$ with the scalar $c$ equal to that of $V$ :

$$
\left[L_{m}, L_{n}\right]=(m-n) L_{m+n}+\frac{1}{12}\left(m^{3}-m\right) \delta_{m+n, 0} c,
$$

for $m, n \in \mathbf{Z}$, where

$$
\begin{gathered}
Y_{M}(\omega, z)=\sum_{n \in \mathbf{Z}} L_{n} z^{-n-2} ; \\
L_{0} v=s v, \text { for } v \in M_{s} ; \\
\frac{d}{d z} Y_{M}(a, z)=Y_{M}\left(L_{-1} a, z\right) .
\end{gathered}
$$

This completes the definition.

(1.2.20) has the same meaning as (1.2.7). It is clear that the $V$ itself is a representation of $V$, we call it the adjoint representation or 0-sector.

Relations (1.2.11)-(1.2.14) hold also for strong modules. In particular, we have

$$
a(n) M_{s} \subset M_{s+\operatorname{deg} a-1-n},
$$

for a homogeneous $a \in V$. This implies that for $s$ satisfying $M_{s-n}=0$ for every positive integer $n, M(s)=\bigoplus_{n=0}^{\infty} M_{s+n}$ is a strong subrepresentation for $V$. Let $S$ be the set of such $s$, then $M$ is a direct sum of strong subrepresentations $M=$ $\bigoplus_{s \in S} M(s)$.

Definition 1.2.3. A representation or a module for $V$ is a graded vector space $M=\bigoplus_{n \in \mathbf{Z}} M_{n}$ such that $M_{n}=0$ for $n$ sufficiently small and $M$ is equipped with a linear map

$$
\begin{aligned}
& V \rightarrow(\text { End } M)\left[\left[z, z^{-1}\right]\right], \\
& a \mapsto Y_{M}(a, z)=\sum_{n \in \mathbf{Z}} a(n) z^{-n-1},
\end{aligned}
$$


such that (1.2.18)-(1.2.23) except (1.2.22) hold, and (1.2.22) is replaced by the following weaker condition: for a homogeneous $a \in V$,

$$
a(n) M_{m} \subset M_{m+\operatorname{deg} a-1-n} .
$$

This completes the definition.

Although the categorical concepts on the representations are not essentially used in this work, for completeness, we include here the necessary definitions to make the modules of $V$ a category. A morphism from $M=\bigoplus_{n \in \mathbf{Z}} M_{n}$ to $N=\bigoplus_{n \in \mathbf{Z}} N_{n}$ is a linear map $f: M \rightarrow N$ such that $f(a(n) v)=a(n) f(v)$ for all $v \in M, a \in V$ and $n \in \mathbf{Z}$ and $f$ shifts the gradations by a fixed integer, i.e., there is an integer $k$ such that $f\left(M_{n}\right) \subset N_{n+k}$ for all $n$. $M$ and $N$ are isomorphic if there are morphisms $f: M \rightarrow N$ and $g: N \rightarrow M$ such that $f g=1$ and $g f=1$. Note that for a given $M=\bigoplus_{n \in \mathbf{Z}} M_{n}$, if we shift the gradation by an integer, i.e. set $M_{n}^{\prime}=M_{n+k}$, the representation $M^{\prime}=\sum_{n \in \mathbf{Z}} M_{n}^{\prime}$ is in the same isomorphism class as $M$. In the following we will only deal with the representations $M=\bigoplus_{n \in \mathbf{Z}} M_{n}$ such that $M_{n}=0$ for $n<0$ and $M_{0} \neq 0$, and we write it as $M=\bigoplus_{n=0}^{\infty} M_{n}$.

If a morphism $f: W \rightarrow M$ is injective, we call the image of $W$ a subrepresentation of $M . M$ is irrducible if it has no subrepresentations other than 0 and $M$ itself.

To see that a strong representation $M=\bigoplus_{s \in \mathbf{C}} M_{s}$ is a representation, we reorganize the gradation of $M$ as follows. We set $M[n]=\bigoplus_{s \in S} M_{s+n}$ (where the set $S$ is defined as above); then $M=\sum_{n=0}^{\infty} M[n]$ is the gradation for a representation.

It is clear that (1.2.11)-(1.2.14) hold for representations.

Since for given $a \in V$ and $v \in M, a(n) v=0$ for $n$ sufficiently large, for a Laurent power series $f(z)=\sum_{i \geq N}^{\infty} l_{i} z^{i}$, the operator

$$
\operatorname{Res}_{z}(Y(a, z) f(z))=\sum_{i \geq N}^{\infty} l_{i} a(i)
$$

is a well-defined operator on $M$.

Lemma 1.2.1. If $M=\bigoplus_{n=0}^{\infty} M_{n}$ is an irreducible representation for $V$, and $M_{0} \neq 0$ has countable dimension, then there is a constant $h$ such that $L_{0}$ acts on each $M_{n}$ as the scalar $n+h$. In particular, $M$ is a strong representation for $V$.

This lemma is a slight generalization of Dixmier's Lemma for representations of algebras (cf. [W], p. 11); the proof is also similar.

Sketch of Proof. Since $M$ is irreducible, $M$ is generated by $M_{0}$ (i.e., every element of $M$ is a linear combination of the elements $a_{1}\left(n_{1}\right) \cdot \ldots \cdot a_{k}\left(n_{k}\right) v$, where $a_{i} \in V$, $\left.v \in M_{0}\right)$. It is sufficient to prove that $L_{0}$ acts on $M_{0}$ as $h I$ for some constant $h \in \mathbf{C}$.

Note that $L_{0}$ preserves $M_{0}$. Since $M_{0}$ has countable dimension, there exists $h \in \mathbf{C}$ such that $L_{0}-h I$ is not invertible on $M_{0}$ (cf. [W], page 10). So at least one of the spaces $\left.\operatorname{Ker}\left(L_{0}-h I\right)\right|_{M_{0}}$ and $\left.\operatorname{Im}\left(L_{0}-h I\right)\right|_{M_{0}}$ is a proper subspace of $M_{0}$. Let $M^{\prime}=\bigoplus_{n=0}^{\infty} M_{n}^{\prime}$ and $M^{\prime \prime}=\bigoplus_{n=0}^{\infty} M_{n}^{\prime \prime}$ be the subrepresentations generated by $\left.\operatorname{Ker}\left(L_{0}-h I\right)\right|_{M_{0}}$ and $\left.\operatorname{Im}\left(L_{0}-h I\right)\right|_{M_{0}}$ respectively. Using commutation relations (1.2.11) and the fact that operators $a(\operatorname{deg} a-1)$ for $a \in V$ homogeneous commute with $L_{0}$, one can prove that $M_{0}^{\prime}=\left.\operatorname{Ker}\left(L_{0}-h I\right)\right|_{M_{0}}$ and $M_{0}^{\prime \prime}=\left.\operatorname{Im}\left(L_{0}-h I\right)\right|_{M_{0}}$. These facts together with the irreducibility of $M$ imply that $L_{0}=h I$ on $M_{0}$.

An immediate corollary of Lemma 1.2.1 is the following: 
Lemma 1.2.2. If $V$ has countable basis and $M=\bigoplus_{n=0}^{\infty} M_{n}$ is an irreducible representation of $V$, then $L_{0}$ acts on each $M_{0}$ as a scalar $n+h$ for some constant $h$. In particular, $M$ is a strong representation of $V$.

An important class of vertex operator algebras is the class of rational vertex operator algebras, which are defined as follows:

Definition 1.2.4. A vertex operator algebra is rational if it has only finitely many isomorphism classes of irreducible modules, and each irreducible module $M=\bigoplus_{n \in \mathbf{N}} M_{n}$ satisfies $\operatorname{dim}\left(M_{n}\right)<\infty$; and moreover every module is a direct sum of irreducible ones.

By Lemma 1.2.1, every irreducible representation of a rational vertex operator algebra is an irreducible strong representation. We see that there is no difference between strong representations and representations for rational vertex operator algebras.

The strong representations defined here are called representations in [FLM2] and [FHL]. The reason we adopt Definition 1.2.3 is for convenience in stating the theorems in Section 2.

1.3. Examples of vertex operator algebras. One of the most striking examples of vertex operator algebras is the Moonshine module [FLM2] (denoted there by $V^{\natural}$ ). $V^{\natural}$ has the Monster sporadic group as its automorphism group. The vertex operator algebra structure of $V^{\natural}$ induces the Greiss algebra structure on the homogeneous subspace $V_{2}^{\natural}$. It is proved in [D] that $V^{\natural}$ is rational and the adjoint representation is the only irreducible representation. As a graded vector space and a representation of the Monster group, $V^{\natural}$ was constructed in [FLM1], and a part of the vertex operator map $a \mapsto Y(a, z)$ was also constructed there. It was realized in [Bo] that $V^{\natural}$ has the structure of a vertex operator algebra, and the first version of the axioms of vertex operator algebras was introduced (they were called vertex algebras in $[\mathrm{Bo}])$.

Associated with each positive definite integral even lattice $L$, there is a vertex operator algebra $V_{L}$ [Bo] [FLM2]. Let $\mathbf{C}(L)$ be the group algebra of the additive group $L$, put $L_{\mathbf{C}}=\mathbf{C} \otimes_{\mathbf{z}} L$, and let $L_{-}=\bigoplus_{i \geq 1} L_{i}$ be the direct sum of infinite copies of $L_{\mathbf{C}}$ and $S\left(L_{-}\right)$the symmetric algebra of the vector space $L_{-}$. Then $V_{L}=S\left(L_{-}\right) \otimes \mathbf{C}(L)$ has a vertex operator algebra structure. See [FLM2] for detailed expositions. $V_{L}$ is rational, and its irreducible representations are in oneto-one correspondence to the elements of the quotient group $L^{\prime} / L$, where $L^{\prime}$ is the dual lattice of $L$. See [D2] for a proof of these facts.

Another infinite family of vertex operator algebras is constructed from affine Kac-Moody algebras. Let $\mathfrak{g}$ be an $n$-dimensional complex simple Lie algebra, $\hat{\mathfrak{g}}$ its associated Kac-Moody affine Lie algebra, and $\left\{\Lambda_{0}, \Lambda_{1}, \ldots, \Lambda_{n}\right\}$ the fundamental weights of $\hat{\mathfrak{g}}$ such that $\left\{\Lambda_{1}, \ldots, \Lambda_{n}\right\}$ are the fundamental weights of $\mathfrak{g}$. Let $M_{k \Lambda_{0}}$ be the Verma module of $\hat{\mathfrak{g}}$ with the highest weight $k \Lambda_{0}$ and highest weight vector 1 , where $k$ is any complex number. Let $M_{k}$ be the quotient module of $M_{k \Lambda_{0}}$ with the relations $a 1=0$ for $a \in \mathfrak{g}$. It is proved in [FZ] that $M_{k}$ has a structure of vertex operator algebra for $k$ different from $-g$,the negative dual Coxeter number of $\mathfrak{g}$. Its identity element can be chosen to be the highest weight vector 1, and its Virasoro element is given by the Sugawara-Segal construction. In the critical level $-g$, $M_{-g \Lambda}$ satisfies all the axioms of vertex operator algebras except the axiom on Virasoro algebra. 
Every $\hat{\mathfrak{g}}$-submodule of $M_{k}$ is an ideal as a vertex operator algebra, so a proper quotient $\hat{\mathfrak{g}}$-module of $M_{k}(k \neq-g)$ has an induced vertex operator algebra structure. In particular, the irrducible highest weight modules $L_{k \Lambda_{0}}(k \neq-g)$ are vertex operator algebras. It was proved in [FZ] that when $k$ is a positive integer, $L_{k \Lambda_{0}}$ is rational; its irreducible representations are precisely the irreducible integral highest weight $\hat{\mathfrak{g}}$-modules of level $k$. See [Li] for the generalization to finite dimensional Lie algebras with a non-degenerate associative bilinear form.

Similarly, for the Virasoro algebra, let $M_{c, h}$ be the Verma module with central charge $c$ and highest level $h$, let $M_{c}$ be the quotient module of $M_{c, h}$ by the relation $L_{-1} 1=0$ (where 1 denotes a highest weight vector of $M_{c, h}$ ); then $M_{c}$ is a vertex operator algebra with 1 as the identity element and $L_{-2} 1$ as the Virasoro element [FZ]. Every submodule $I$ of $M_{c}$ is an ideal, so the quotient module $M_{c} / I$ with $L_{-2} 1 \neq 0$ is also a vertex operator algebra. In particular, the irrducible highest weight modules $L_{c, 0}$ for $c \neq 0$ are vertex operator algebras. The irreducible representations of $L_{c, 0}$ were completely classified in [Wa]. In particular, it was proved in [Wa] that $L_{c, 0}$ is rational if and only if $c=1-6(p-q)^{2} / p q$, where $p, q \in\{2,3,4, \ldots\}$ and $p, q$ are relatively prime. The irreducible $L(c, 0)$-modules for $c=1-6(p-q)^{2} / p q$ as above are precisely the minimal series with the same central charge $1-6(p-q)^{2} / p q$.

This list is far from being complete. For example, see [BS] [FFr] [F] [FKRW] and the references there for an important class of vertex operator algebras called $W$-algebras.

\section{Associative algebras attached to vertex operator algebras}

We construct an associative algebra $A(V)$ from a vertex operator algebra $V$. $A(V)$ is a quotient space of $V$, and its multiplication law is induced from the vertex operators of $V$. It enjoys the property that the top level $M_{0}$ of a representation $M=\bigoplus_{n=0}^{\infty} M_{n}$ has a structure of $A(V)$-module (recall our convention, $M$ is so graded that $\left.M_{0} \neq 0\right)$; and conversely, for an $A(V)$-module $W$, there exists a representation of $V$ such that its top level is isomorphic to $W$ as an $A(V)$-module; and the isomorphism classes of the irreducible $A(V)$-modules and the isomorphism classes of the irreducible representations for $V$ are in one-to-one correspondence. $A(V)$ plays an important role in our proof of modular invariance in Section 5.

2.1. The associative algebra $A(V)$. Recall that for a representation $M=$ $\bigoplus_{n=0}^{\infty} M_{n}$ of $V$ and a homogeneous element $a \in V$, the operator $a(\operatorname{deg} a-1)$ preserves the degree of $M$. The study of the action of operators $a(\operatorname{deg} a-1)$ on the top levels of representations leads naturally the construction of $A(V)$.

Definition 2.1.1. Define $o(a)=a(\operatorname{deg} a-1)$ for $a \in V$ homogeneous, and for general $a \in V$, we define $o(a)$ by linearity.

Definition 2.1.2. We define a bilinear operation $*$ on $V$ as follows, for $a$ homogeneous:

$$
a * b=\operatorname{Res}_{z}\left(Y(a, z) \frac{(z+1)^{\operatorname{deg} a}}{z} b\right) .
$$

And we denote by $O(V)$ of the subspace of $V$ spanned by elements

$$
\operatorname{Res}_{z}\left(Y(a, z) \frac{(z+1)^{\operatorname{deg} a}}{z^{2}} b\right),
$$


and by $A(V)$ the quotient space $V / O(V)$.

The space $O(V)$ is characterized by the property that for $a \in O(V)$, the action of $o(a)$ on the top level $M_{0}$ of $M$ is 0 . And the multiplication $*$ satisfies $\left.o(a) o(b)\right|_{M_{0}}=$ $\left.o(a * b)\right|_{M_{0}}$. Before proving these facts we first prove that $A(V)$ is an associative algebra.

The expression $(1+z)^{k}\left(\right.$ similarly $\left.(1+w)^{k}\right)$ in the rest of Section 2 means the power series $\sum_{i=0}^{\infty}\left(\begin{array}{c}k \\ i\end{array}\right) z^{i}$.

Theorem 2.1.1. $O(V)$ is an two sided ideal for the multiplication $*$, so $*$ is defined on $A(V)$. Moreover:

(1) $A(V)$ is an associative algebra under multiplication *.

(2) The image of the vacuum 1 is the unit of the algebra $A(V)$.

(3) The image of $\omega$ is in the center of $A(V)$.

(4) $A(V)$ has a filtration $A_{0}(V) \subset A_{1}(V) \subset \ldots$, where $A_{n}(V)$ is the image of $\bigoplus_{i=0}^{n} V_{i}$.

We need some lemmas to prove this theorem.

Lemma 2.1.1. $L_{-1} a+L_{0} a \in O(V)$ for every $a \in V$.

Proof. Because

$$
L_{-1} a+L_{0} a=\operatorname{Res}_{z}\left(Y(a, z) \frac{(z+1)^{\operatorname{deg} a}}{z^{2}} 1\right) \in O(V) .
$$

Lemma 2.1.2. For every homogeneous element $a \in V$, and $m \geq n \geq 0$

$$
\operatorname{Res}_{z}\left(Y(a, z) \frac{(z+1)^{\operatorname{deg} a+n}}{z^{2+m}} b\right) \in O(V) .
$$

Proof. Since

$$
\frac{(z+1)^{\operatorname{deg} a+n}}{z^{2+m}}=\sum_{i=0}^{n}\left(\begin{array}{c}
n \\
i
\end{array}\right) \frac{(z+1)^{\operatorname{deg} a}}{z^{2+m-i}}
$$

we only need to prove the lemma for the case $n=0$ and $m \geq 0$. Use induction on $m$. The case $m=0$ follows from the definition of $O(V)$. Assume that in the case $m \leq k$ the lemma is true, then for $m=k+1$, by the the induction assumption, we have

$$
\operatorname{Res}_{z}\left(Y\left(L_{-1} a, z\right) \frac{(z+1)^{\operatorname{deg} a+1}}{z^{2+k}} b\right) \in O(V) ;
$$

on the other hand,

$$
\begin{aligned}
\operatorname{Res}_{z}\left(Y\left(L_{-1} a, z\right) \frac{(z+1)^{\operatorname{deg} a+1}}{z^{2+k}} b\right) \\
=\operatorname{Res}_{z}\left(\frac{d}{d z} Y(a, z) \frac{(z+1)^{\operatorname{deg} a+1}}{z^{2+k}} b\right) \\
=-\operatorname{Res}_{z}\left(Y(a, z) \frac{d}{d z} \frac{(z+1)^{\operatorname{deg} a+1}}{z^{2+k}} b\right) \\
=-(\operatorname{deg} a+1) \operatorname{Res}_{z}\left(Y(a, z) \frac{(z+1)^{\operatorname{deg} a}}{z^{2+k}} b\right) \\
\quad+(2+k) \operatorname{Res}_{z}\left(Y(a, z) \frac{(z+1)^{\operatorname{deg} a}}{z^{2+k+1}} b\right),
\end{aligned}
$$


and since the left side and the first term on the right hand side are in $O(V)$, so is the second term on the right hand side.

Lemma 2.1.3. If $a$ and $b$ are homogeneous elements of $V$, then we have the identities

$$
\begin{gathered}
a * b \equiv \operatorname{Res}_{z}\left(Y(b, z) \frac{(z+1)^{\operatorname{deg} b-1}}{z} a\right) \quad \bmod O(V), \\
a * b-b * a \equiv \operatorname{Res}_{z}\left(Y(a, z)(z+1)^{\operatorname{deg} a-1} b\right) \quad \bmod O(V) .
\end{gathered}
$$

Proof. We first prove the identity:

$$
Y(a, z) b \equiv(z+1)^{-\operatorname{deg} a-\operatorname{deg} b} Y\left(b, \frac{-z}{z+1}\right) a \quad \bmod O(V)
$$

We need the identity $Y(a, z) b=e^{z L_{-1}} Y(b,-z) a$, which is a direct corollary of Definition 1.2.1 (see also [FHL] for a proof). We have

$$
\begin{aligned}
& Y(a, z) b=e^{z L-1} Y(b,-z) a \\
& =\sum_{i \in \mathbf{N}} e^{z L_{-1}} b(i) a(-z)^{-i-1} \\
& \equiv \sum_{i=0}^{\infty}(-1)^{i+1} b(i) a(z+1)^{-\operatorname{deg} a-\operatorname{deg} b+i+1} z^{-i-1} \bmod O(V) \quad \text { using Lemma }(2.1 .1) \\
& =(z+1)^{-\operatorname{deg} a-\operatorname{deg} b} Y\left(b, \frac{-z}{z+1}\right) a
\end{aligned}
$$

Using (2.1.5), we have

$$
\begin{aligned}
& a * b=\operatorname{Res}_{z}\left(Y(a, z) \frac{(z+1)^{\operatorname{deg} a}}{z} b\right) \\
& \equiv \operatorname{Res}_{z}\left((z+1)^{-\operatorname{deg} a-\operatorname{deg} b} Y\left(b, \frac{-z}{z+1}\right) \frac{(z+1)^{\operatorname{deg} a}}{z} a\right) \\
& =\operatorname{Res}_{w}\left(Y(b, w) \frac{(w+1)^{\operatorname{deg} b-1}}{w} a\right)
\end{aligned}
$$

in the last step, we changed the variable $w=\frac{-z}{1+z}$ and used (1.3.1). This proves (2.1.3). (2.1.4) follows directly from the definition of $*$ and (2.1.3).

Proof of Theorem 2.1.1. Proving that $O(V)$ is a two sided ideal and the quotient space $A(V)$ is an associative algebra under multiplication $*$ is equivalent to proving the following relations for homogeneous elements $a, b$ and $c$ :

$$
\begin{aligned}
& a * O(V) \subset O(V), \\
& O(V) * a \subset O(V), \\
& (a * b) * c-a *(b * c) \in O(V) .
\end{aligned}
$$


To prove (2.1.6), we have

$$
\begin{aligned}
& \operatorname{Res}_{z}\left(Y(a, z) \frac{(z+1)^{\operatorname{deg} a}}{z}\right) \operatorname{Res}_{w}\left(Y(b, w) \frac{(w+1)^{\operatorname{deg} b}}{w^{2}}\right) c \\
& -\operatorname{Res}_{w}\left(Y(b, w) \frac{(w+1)^{\operatorname{deg} b}}{w^{2}}\right) \operatorname{Res}_{z}\left(Y(a, z) \frac{(z+1)^{\operatorname{deg} a}}{z}\right) c \\
& =\operatorname{Res}_{w} \operatorname{Res}_{z-w}\left(Y(Y(a, z-w) b, w) \frac{(z+1)^{\operatorname{deg} a}}{z} \frac{(w+1)^{\operatorname{deg} b}}{w^{2}} c\right) \\
& =\sum_{i=0}^{\operatorname{deg} a} \sum_{j=0}^{\infty}\left(\begin{array}{c}
\operatorname{deg} a \\
i
\end{array}\right) \operatorname{Res}_{w}\left(Y(a(i+j) b, w)(-1)^{j} \frac{(w+1)^{\operatorname{deg} a+\operatorname{deg} b-i}}{w^{j+3}}\right) c .
\end{aligned}
$$

Since $\operatorname{deg}(a(i+j) b)=\operatorname{deg} a+\operatorname{deg} b-i-j-1$, by Lemma 2.1.2, the right hand side of the last identity is in $O(V)$, and the second term of the left hand side is in $O(V)$; then so is the first term. This proves (2.1.6). The proof of (2.1.7) is similar to the proof of (2.1.8) given below, so we omit it.

For $(2,1.8)$, we have

$$
\begin{aligned}
& (a * b) * c \\
= & \sum_{i=0}^{\operatorname{deg} a}\left(\begin{array}{c}
\operatorname{deg} a \\
i
\end{array}\right)(a(i-1) b) * c \\
= & \sum_{i=0}^{\operatorname{deg} a}\left(\begin{array}{c}
\operatorname{deg} a \\
i
\end{array}\right) \operatorname{Res}_{w}\left(Y(a(i-1) b, w) \frac{(w+1)^{\operatorname{deg} a+\operatorname{deg} b-i}}{w} c\right) \\
= & \left.\sum_{i=0}^{\operatorname{deg} a}\left(\begin{array}{c}
\operatorname{deg} a \\
i
\end{array}\right) \operatorname{Res}_{w} \operatorname{Res}_{z-w}(Y(a, z-w) b, w)(z-w)^{i-1} \frac{(w+1)^{\operatorname{deg} a+\operatorname{deg} b-i}}{w} c\right) \\
= & \left.\operatorname{Res}_{w} \operatorname{Res}_{z-w}(Y(a, z-w) b, w) \frac{(z+1)^{\operatorname{deg} a}(w+1)^{\operatorname{deg} b}}{w(z-w)} c\right) \\
= & \operatorname{Res}_{z} \operatorname{Res}_{w}\left(Y(a, z) Y(b, w) \frac{(z+1)^{\operatorname{deg} a}(w+1)^{\operatorname{deg} b}}{w(z-w)} c\right) \\
& -\operatorname{Res}_{w} \operatorname{Res}_{z}\left(Y(b, w) Y(a, z) \frac{(z+1)^{\operatorname{deg} a}(w+1)^{\operatorname{deg} b}}{w(z-w)} c\right) \\
= & \sum_{i=0}^{\infty} \operatorname{Res}_{z} \operatorname{Res}_{w}\left(Y(a, z) Y(b, w)(-1)^{i} z^{-1-i} w^{i} \frac{(z+1)^{\operatorname{deg} a}(w+1)^{\operatorname{deg} b}}{w} c\right) \\
& -\sum_{i=0}^{\infty} \operatorname{Res}_{w} \operatorname{Res}_{z}\left(Y(b, w) Y(a, z)(-1)^{i+1} w^{-1-i} z^{i} \frac{(z+1)^{\operatorname{deg} a}(w+1)^{\operatorname{deg} b}}{w} c\right) \\
\equiv & \operatorname{Res}_{z} \operatorname{Res}_{w}\left(Y(a, z) Y(b, w) \frac{(z+1)^{\operatorname{deg} a}(w+1)^{\operatorname{deg} b}}{z w} m\right) \bmod (O(V)) \\
= & a *(b * c) .
\end{aligned}
$$

This completes the proof of statement (1).

Statement (2) is trivial. 
Concerning (3), by 2.1.4, we have

$$
\begin{aligned}
& \omega * a-a * \omega \\
= & \operatorname{Res}_{z}\left(Y(\omega, z) \frac{(z+1)^{1}}{z} a\right)=L_{-1} a+L_{0} a \in O(V) .
\end{aligned}
$$

This proves (3).

If $a \in V_{m}$ and $b \in V_{n}$, then $a * b \in \bigoplus_{i=0}^{m+n} V_{i}$. This proves (4).

$A(V)$ has an involution ' defined by

$$
a^{\prime}=e^{L_{1}}(-1)^{L_{0}} a .
$$

This can proved by using the identity

$$
e^{z_{0} L_{1}} Y(a, z) e^{-z_{0} L_{1}}=Y\left(e^{z_{0}\left(1-z_{0} z\right) L_{1}}\left(1-z_{0} z\right)^{-2 L_{0}} a, \frac{z}{1-z_{0} z}\right),
$$

which is proved in $[\mathrm{FLH}]$. Since this fact will not be used, we omit the detailed proof.

Theorem 2.1.2. If $M=\bigoplus_{n=0}^{\infty} M_{n}$ is a representation of $V$, then the top level of $M_{0}$ is a representation of the associative algebra $A(V)$. The action is given as follows: for $(a) \in A(V)$ which is the image of $a \in V,(a)$ acts on $M_{0}$ as o(a).

Proof. It suffices to prove that, for $a, b \in V,\left.o(a) o(b)\right|_{M_{0}}=o(a * b) \mid{ }_{M_{0}}$, and for $c \in O(V),\left.o(c)\right|_{M_{0}}=0$. Note that the top level $M_{0}$ has the property

$$
\left.a(\operatorname{deg} a-1+n)\right|_{M_{0}}=\left.b(\operatorname{deg} b-1+n)\right|_{M_{0}}=0
$$

for $n>0$. Using (2.1.10), we have, in End $M_{0}$,

$$
\begin{aligned}
o(a * b)=\sum_{i=0}^{\operatorname{deg} a}\left(\begin{array}{c}
\operatorname{deg} a \\
i
\end{array}\right) o(a(i-1) b) \\
=\sum_{i=0}^{\operatorname{deg} a}\left(\begin{array}{c}
\operatorname{deg} a \\
i
\end{array}\right)(a(i-1) b)(\operatorname{deg} a+\operatorname{deg} b-i-1) \\
=\operatorname{Res}_{w} \operatorname{Res}_{z-w} \sum_{i=0}^{\operatorname{deg} a}\left(\begin{array}{c}
\operatorname{deg} a \\
i
\end{array}\right)(z-w)^{i-1} w^{\operatorname{deg} a+\operatorname{deg} b-i-1} Y((a, z-w) b, w) \\
=\operatorname{Res}_{w} \operatorname{Res}_{z-w} Y((a, z-w) b, w) \iota_{w, z-w}\left(\frac{z^{\operatorname{deg} a} w^{\operatorname{deg} b-1}}{z-w}\right) \\
=\operatorname{Res}_{z} \operatorname{Res}_{w} Y(a, z) Y(b, w) \iota_{z, w}\left(\frac{z^{\operatorname{deg} a} w^{\operatorname{deg} b-1}}{z-w}\right) \\
-\operatorname{Res}_{w} \operatorname{Res}_{z} Y(b, w) Y(a, z) \iota_{w, z}\left(\frac{z^{\operatorname{deg} a} w^{\operatorname{deg} b-1}}{z-w}\right) \quad(\text { use } 1.2 .20) \\
=\sum_{i \in \mathbf{N}} \operatorname{Res}_{z} \operatorname{Res}_{w} z^{\operatorname{deg} a-i-1} w^{\operatorname{deg} b-1+i} Y(a, z) Y(b, w)
\end{aligned}
$$




$$
\begin{aligned}
& -\sum_{i \in \mathbf{N}} \operatorname{Res}_{w} \operatorname{Res}_{z} z^{\operatorname{deg} a+i} w^{\operatorname{deg} b-2-i} Y(b, w) Y(a, z) \\
= & o(a) o(b),
\end{aligned}
$$

This proves $o(a) o(b)=o(a * b)$ in End $M_{0}$.

By (1.2.12), we have $o\left(L_{-1} a\right)+\operatorname{deg} a \cdot o(a)=0$, thus $o\left(\left(L_{-1} a\right)+\operatorname{deg} a \cdot a\right) o(b)=0$. Using the relation $\left.o(x) o(b)\right|_{M_{0}}=\left.o(x * b)\right|_{M_{0}}$ just proved, we have

$$
o\left(\left(L_{-1} a\right) * b+\operatorname{deg} a \cdot a * b\right)=0 \text { in End } M_{0} .
$$

It is easy to show that

$$
\left.\left(L_{-1} a\right) * b+\operatorname{deg} a \cdot a * b\right)=\operatorname{Res}_{z}\left(Y(a, z) \frac{(z+1)^{w t a}}{z^{2}} b\right) .
$$

This proves that $\left.o(c)\right|_{M_{0}}=0$ for $c \in O(V)$.

2.2. Correspondence of $V$-modules and $A(V)$-modules. In this subsection, we prove a converse of Theorem 2.1.2.

Theorem 2.2.1. If $\pi: A(V) \rightarrow$ End $W$ is representation of associative algebra $A(V)$, then there exists a representation $M=\bigoplus_{i=0}^{\infty} M_{n}$ of $V$ such that $M_{0}=W$ as a $A(V)$-module and every subrepresentation $N=\bigoplus_{i=0}^{\infty} N_{n}$ of $M$ satisfying $N_{0}=$ $\{0\}$ is $\{0\}$.

Before proving this theorem, we derive a recurrent formula for $(n+2)$-point correlation functions on the sphere which motivates our proof.

Lemma 2.2.1. Let $M=\bigoplus_{n \in \mathbf{N}} M_{n}$ be a representation of $V, M^{\prime}=\bigoplus_{n \in \mathbf{N}} M_{n}^{*}$ its restricted dual. If $v \in M_{0}, v^{\prime} \in M_{0}^{*}$, and $a_{1}$ is homogeneous in $V$, then

$$
\begin{aligned}
& \left(v^{\prime}, Y\left(a_{1}, z_{1}\right) Y\left(a_{2}, z_{2}\right) \cdots Y\left(a_{n}, z_{n}\right) v\right) \\
& =\sum_{k=2}^{n} \sum_{i=0}^{\infty} F_{\operatorname{deg} a_{1}, i}\left(z_{1}, z_{k}\right)\left(v^{\prime}, Y\left(a_{2}, z_{2}\right) \cdots Y\left(a_{1}(i) a_{k}, z_{k}\right) \cdots Y\left(a_{n}, z_{n}\right) v\right) \\
& \quad+z_{1}^{-\operatorname{deg} a_{1}}\left(a_{1}\left(\operatorname{deg} a_{1}-1\right)^{*} v^{\prime}, Y\left(a_{2}, z_{2}\right) \cdots Y\left(a_{n}, z_{n}\right) v\right),
\end{aligned}
$$

where $F_{\operatorname{deg} a, i}$ is a rational function defined by

$$
\begin{aligned}
& \iota_{z, w} F_{n, i}(z, w)=\sum_{j \in \mathbf{N}}\left(\begin{array}{c}
n+j \\
i
\end{array}\right) z^{-n-j-1} w^{n+j-i}, \\
& F_{n, i}(z, w)=\frac{z^{-n}}{i !}\left(\frac{d}{d w}\right)^{i} \frac{w^{n}}{z-w} .
\end{aligned}
$$

Proof. For $a \in V$ homogeneous, since $a(n) M_{m} \subset M_{m+\operatorname{deg} a-n-1}$, we have

$$
\begin{aligned}
& a(\operatorname{deg} a-1+n) v=0 \quad \text { for } n>0, v \in M_{0}, \\
& a_{1}^{*}(\operatorname{deg} a-1-n) v^{\prime}=0 \quad \text { for } n>0, v^{\prime} \in M_{0}^{*},
\end{aligned}
$$


where $a^{*}(m)$ denotes the adjoint operator of $a(m)$.

By (2.2.3) and (2.2.4), we have

$$
\begin{aligned}
\left\langle v^{\prime},\right. & \left.Y\left(a_{1}, z_{1}\right) Y\left(a_{2}, z_{2}\right) \ldots Y\left(a_{m}, z_{m}\right) v\right\rangle \\
= & \left\langle v^{\prime}, \sum_{n \in \mathbf{Z}} a_{1}\left(\operatorname{deg} a_{1}-1+n\right) z_{1}^{-\operatorname{deg} a_{1}+1-n} Y\left(a_{2}, z_{2}\right) \ldots Y\left(a_{m}, z_{m}\right) v\right\rangle \\
= & \left\langle v^{\prime}, \sum_{n>0} a_{1}\left(\operatorname{deg} a_{1}-1-n\right) z_{1}^{-\operatorname{deg} a_{1}+1+n} Y\left(a_{2}, z_{2}\right) \ldots Y\left(a_{m}, z_{m}\right) v\right\rangle \\
& +\left\langle v^{\prime}, \sum_{n>0} a_{1}\left(\operatorname{deg} a_{1}-1+n\right) z_{1}^{-\operatorname{deg} a_{1}+1-n} Y\left(a_{2}, z_{2}\right) \ldots Y\left(a_{m}, z_{m}\right) v\right\rangle \\
& +\left\langle v^{\prime}, a_{1}\left(\operatorname{deg} a_{1}-1\right) z_{1}^{-\operatorname{deg} a_{1}} Y\left(a_{2}, z_{2}\right) \ldots Y\left(a_{m}, z_{m}\right) v\right\rangle \\
= & \left\langle v^{\prime}, \sum_{n>0} a_{1}\left(\operatorname{deg} a_{1}-1+n\right) z_{1}^{-\operatorname{deg} a_{1}+1-n} Y\left(a_{2}, z_{2}\right) \ldots Y\left(a_{m}, z_{m}\right) v\right\rangle \\
& +\left\langle v^{\prime}, a_{1}\left(\operatorname{deg} a_{1}-1\right) z_{1}^{-\operatorname{deg} a_{1}} Y\left(a_{2}, z_{2}\right) \ldots Y\left(a_{m}, z_{m}\right) v\right\rangle \\
= & \sum_{k=2}^{m}\left\langle v^{\prime}, Y\left(a_{2}, z_{2}\right) \ldots\left[\sum_{n>0} a_{1}\left(\operatorname{deg} a_{1}-1+n\right) z_{1}^{-\operatorname{deg} a_{1}-n}, Y\left(a_{k}, z_{k}\right)\right] \ldots Y\left(a_{m}, z_{m}\right) v\right\rangle \\
& +\left\langle v^{\prime}, a_{1}\left(\operatorname{deg} a_{1}-1\right) z_{1}^{-\operatorname{deg} a_{1}} Y\left(a_{2}, z_{2}\right) \ldots Y\left(a_{n}, z_{n}\right) v\right\rangle \\
= & \sum_{k=2}^{n} \sum_{i \in \mathbf{N}} \iota_{z_{1}, z_{k}} F_{\operatorname{deg} a_{1}, i}\left(z_{1}, z_{k}\right)\left\langle v^{\prime}, Y\left(a_{2}, z_{2}\right) \ldots Y\left(a_{1}(i) a_{k}, z_{k}\right) \ldots Y\left(a_{n}, z_{n}\right) v\right\rangle \\
& +\left\langle v^{\prime}, a_{1}\left(\operatorname{deg} a_{1}-1\right) z_{1}^{-\operatorname{deg} a_{1}} Y\left(a_{2}, z_{2}\right) \ldots Y\left(a_{n}, z_{n}\right) v\right\rangle . \\
& \\
&
\end{aligned}
$$

This proves (2.2.1).

We see from Lemma 2.2.1 that for $v, v^{\prime}$ in the top level of $M$ and $M^{\prime}$ respectively, the correlation functions $\left(v^{\prime}, Y\left(a_{1}, z_{1}\right) Y\left(a_{2}, z_{2}\right) \ldots Y\left(a_{m}, z_{m}\right) v\right)$ depend only on the structure of $V$ and the action of the operators $o(a)$ on the top level. This observation motivates the following proof.

Proof of Theorem 2.2.1. We will use the same letter $a$ to denote the image of $a \in V$ in $A(V)$. We organize the proof into several steps.

Step 1. Let $W^{*}$ be the dual vector space of $W$. Let $($,$) denote the pairing$ between elements in $W^{*}$ and $W$. Suppose $M$ is a representation with top level $W$. Then by Lemma 2.2.1 we know that, for $v^{\prime} \in W^{*}, v \in W$ and $a_{i} \in V$, the function

$$
\left(v^{\prime}, Y\left(a_{1}, z_{1}\right) Y\left(a_{2}, z_{2}\right) \ldots Y\left(a_{n}, z_{n}\right) v\right)
$$

has an expression which is independent of the higher level of $M$. By this consideration, we define the "(n+2)-point function" $S\left(v^{\prime},\left(a_{1}, z_{1}\right)\left(a_{2}, z_{2}\right) \ldots\left(a_{n}, z_{n}\right) v\right)$ inductively as

$$
S\left(v^{\prime},(a, z) v\right)=z^{-\operatorname{deg} a}\left(v^{\prime}, \pi(a) v\right),
$$




$$
\begin{aligned}
& S\left(v^{\prime},\left(a_{1}, z_{1}\right)\left(a_{2}, z_{2}\right) \ldots\left(a_{m}, z_{m}\right) v\right) \\
= & \sum_{k=2}^{n} \sum_{i=0}^{\infty} F_{\operatorname{deg} a_{1}, i}\left(z_{1}, z_{k}\right) S\left(v^{\prime},\left(a_{2}, z_{2}\right) \ldots\left(a_{1}(i) a_{k}, z_{k}\right) \ldots\left(a_{n}, z_{n}\right) v\right) \\
& +z_{1}^{-\operatorname{deg} a_{1}} S\left(\pi\left(a_{1}\right)^{*} v^{\prime},\left(a_{2}, z_{2}\right) \ldots\left(a_{n}, z_{n}\right) v\right),
\end{aligned}
$$

where $F_{\operatorname{deg} a, i}\left(z_{1}, z_{k}\right)$ is as in (2.2.2).

It is clear that the functions $S$ are rational functions with possible poles only at $z_{i}=0, z_{i}=z_{j}$.

Step 2. We claim that the functions $S\left(v^{\prime},\left(a_{1}, z_{1}\right)\left(a_{2}, z_{2}\right) \ldots\left(a_{m}, z_{m}\right) v\right)$ satisfy the following properties:

$$
\begin{aligned}
& S\left(v^{\prime},\left(a_{1}, z_{1}\right)\left(a_{2}, z_{2}\right) \ldots\left(a_{n}, z_{n}\right) v\right) \\
& =S\left(v^{\prime},\left(a_{i_{1}}, z_{i_{1}}\right)\left(a_{i_{2}}, z_{i_{2}}\right) \ldots\left(a_{i_{n}}, z_{i_{n}}\right) v\right) \\
& S\left(v^{\prime},\left(L_{-1} a_{1}, z_{1}\right)\left(a_{2}, z_{2}\right) \ldots\left(a_{n}, z_{n}\right) v\right) \\
& =\frac{d}{d z_{1}} S\left(v^{\prime},\left(a_{1}, z_{1}\right)\left(a_{2}, z_{2}\right) \ldots\left(a_{n}, z_{n}\right) v\right), \\
& \int_{C} S\left(v^{\prime},\left(a_{1}, z_{1}\right)\left(a_{2}, z_{2}\right) \ldots\left(a_{n}, z_{n}\right) v\right)\left(z_{1}-z_{2}\right)^{k} d z_{1} \\
& =S\left(v^{\prime},\left(a_{1}(k) a_{2}, z_{2}\right)\left(a_{3}, z_{3}\right) \ldots\left(a_{n}, z_{n}\right) v\right),
\end{aligned}
$$

where $\left\{i_{1}, i_{2}, \ldots, i_{n}\right\}$ is an arbitrary permutation of $\{1,2, \ldots, n\}, k$ is an arbitrary integer, and $C$ is a contour of $z_{1}$ surrounding $z_{2}$.

We rewrite (2.2.6) in the form

$$
\begin{aligned}
& S\left(v^{\prime},\left(a_{1}, z_{1}\right)\left(a_{2}, z_{2}\right) \ldots\left(a_{m}, z_{m}\right) v\right) \\
& =z_{1}^{-\operatorname{deg} a_{1}} S\left(\pi\left(a_{1}\right)^{*} v^{\prime},\left(a_{2}, z_{2}\right) \ldots\left(a_{n}, z_{n}\right) v\right) \\
& \quad+\sum_{k=2}^{n} \operatorname{Res}_{w}\left(\frac{z_{1}^{-\operatorname{deg} a_{1}}\left(z_{k}+w\right)^{\operatorname{deg} a_{1}}}{z_{1}-z_{k}-w} S\left(v^{\prime},\left(a_{2}, z_{2}\right) \ldots\left(Y\left(a_{1}, w\right) a_{k}, z_{k}\right) \ldots\left(a_{n}, z_{n}\right) v\right)\right) .
\end{aligned}
$$

Here the term inside of $\operatorname{Res}_{w}$ is a formal Laurent series in $w$ with coefficients as rational functions of $z_{1}, \ldots, z_{n}$, and $\left(z_{k}+w\right)^{\operatorname{deg} a_{1}} /\left(z_{1}-z_{k}-w\right)$ is expanded in positive powers of $w$. (The similar convention applies to formulas (2.2.12), (2.2.15) and (2.2.16) below, where the way to expand rational functions of $w_{1}$ and $w_{2}$ should be clear from the context.)

We want first to prove

$$
S\left(v^{\prime},\left(a_{1}, z_{1}\right)\left(a_{2}, z_{2}\right) \ldots\left(a_{n}, z_{n}\right) v\right)=S\left(v^{\prime},\left(a_{2}, z_{2}\right)\left(a_{1}, z_{1}\right) \ldots\left(a_{n}, z_{n}\right) v\right)
$$

Using recurrent formula (2.2.10) twice for the left-hand side and the right-hand 
side of (2.2.11) respectively, we have

$$
\begin{aligned}
& S\left(v^{\prime},\left(a_{1}, z_{1}\right)\left(a_{2}, z_{2}\right) \ldots\left(a_{n}, z_{n}\right) v\right) \\
& -S\left(v^{\prime},\left(a_{2}, z_{2}\right)\left(a_{1}, z_{1}\right) \ldots\left(a_{n}, z_{n}\right) v\right) \\
& =z_{1}^{-\operatorname{deg} a_{1}} z_{2}^{-\operatorname{deg} a_{2}} S\left(\pi\left(a_{2}\right)^{*} \pi\left(a_{1}\right)^{*} v^{\prime},\left(a_{3}, z_{3}\right) \ldots\left(a_{n}, z_{n}\right) v\right) \\
& -z_{1}^{-\operatorname{deg} a_{1}} z_{2}^{-\operatorname{deg} a_{2}} S\left(\pi\left(a_{1}\right)^{*} \pi\left(a_{2}\right)^{*} v^{\prime},\left(a_{3}, z_{3}\right) \ldots\left(a_{n}, z_{n}\right) v\right) \\
& +\operatorname{Res}_{w_{1}}\left(\frac{z_{1}^{-\operatorname{deg} a_{1}} z_{2}^{-\operatorname{deg} a_{2}+1}\left(1+w_{1}\right)^{\operatorname{deg} a_{1}}}{z_{1}-z_{2}-z_{2} w_{1}}\right. \\
& \left.\cdot S\left(\pi\left(Y\left(a_{1}, w_{1}\right) a_{2}\right)^{*} v^{\prime},\left(a_{3}, z_{3}\right) \ldots\left(a_{n}, z_{n}\right) v\right)\right) \\
& -\operatorname{Res}_{w_{2}}\left(\frac{z_{1}^{-\operatorname{deg} a_{1}+1} z_{2}^{-\operatorname{deg} a_{2}}\left(1+w_{2}\right)^{\operatorname{deg} a_{2}}}{z_{2}-z_{1}-z_{1} w_{2}}\right. \\
& \left.\cdot S\left(\pi\left(Y\left(a_{2}, w_{2}\right) a_{1}\right)^{*} v^{\prime},\left(a_{3}, z_{3}\right) \ldots\left(a_{n}, z_{n}\right) v\right)\right) \\
& +\sum_{k=3}^{n} \operatorname{Res}_{w_{2}} \operatorname{Res}_{w_{1}}\left(\frac{z_{1}^{-\operatorname{deg} a_{1}} z_{2}^{-\operatorname{deg} a_{2}}\left(z_{k}+w_{1}\right)^{\operatorname{deg} a_{1}}\left(z_{k}+w_{2}\right)^{\operatorname{deg} a_{2}}}{\left(z_{1}-z_{k}-w_{1}\right)\left(z_{2}-z_{k}-w_{2}\right)}\right. \\
& \left.\cdot S\left(v^{\prime},\left(a_{3}, z_{3}\right) \ldots\left(Y\left(a_{2}, w_{2}\right) Y\left(a_{1}, w_{1}\right) a_{k}, z_{k}\right) \ldots\left(a_{n}, z_{n}\right) v\right)\right) \\
& -\sum_{k=3}^{n} \operatorname{Res}_{w_{1}} \operatorname{Res}_{w_{2}}\left(\frac{z_{1}^{-\operatorname{deg} a_{1}} z_{2}^{-\operatorname{deg} a_{2}}\left(z_{k}+w_{1}\right)^{\operatorname{deg} a_{1}}\left(z_{k}+w_{2}\right)^{\operatorname{deg} a_{2}}}{\left(z_{1}-z_{k}-w_{1}\right)\left(z_{2}-z_{k}-w_{2}\right)}\right. \\
& \left.\cdot S\left(v^{\prime},\left(a_{3}, z_{3}\right) \ldots\left(Y\left(a_{1}, w_{1}\right) Y\left(a_{2}, w_{2}\right) a_{k}, z_{k}\right) \ldots\left(a_{n}, z_{n}\right) v\right)\right) \\
& +\sum_{k=3}^{n} \operatorname{Res}_{w_{2}} \operatorname{Res}_{w_{1}-w_{2}}\left(\frac{z_{1}^{-\operatorname{deg} a_{1}} z_{2}^{-\operatorname{deg} a_{2}+1}\left(z_{k}+w_{1}\right)^{\operatorname{deg} a_{1}}\left(z_{k}+w_{2}\right)^{\operatorname{deg} a_{2}}}{\left(z_{1}\left(z_{k}+w_{2}\right)-z_{2}\left(z_{k}+w_{1}\right)\right)\left(z_{2}-z_{k}-w_{2}\right)}\right. \\
& \left.\cdot S\left(v^{\prime},\left(a_{3}, z_{3}\right) \ldots\left(Y\left(Y\left(a_{1}, w_{1}-w_{2}\right) a_{2}, w_{2}\right) a_{k}, z_{k}\right) \ldots\left(a_{n}, z_{n}\right) v\right)\right) \\
& -\sum_{k=3}^{n} \operatorname{Res}_{w_{1}} \operatorname{Res}_{w_{2}-w_{1}}\left(\frac{z_{1}^{-\operatorname{deg} a_{1}+1} z_{2}^{-\operatorname{deg} a_{2}}\left(z_{k}+w_{1}\right)^{\operatorname{deg} a_{1}}\left(z_{k}+w_{2}\right)^{\operatorname{deg} a_{2}}}{\left(z_{2}\left(z_{k}+w_{1}\right)-z_{1}\left(z_{k}+w_{2}\right)\right)\left(z_{1}-z_{k}-w_{1}\right)}\right. \\
& \left.\cdot S\left(v^{\prime},\left(a_{3}, z_{3}\right) \ldots\left(Y\left(Y\left(a_{2}, w_{2}-w_{1}\right) a_{1}, w_{1}\right) a_{k}, z_{k}\right) \ldots\left(a_{n}, z_{n}\right) v\right)\right) \text {. }
\end{aligned}
$$

By (2.1.5), we have

$$
\pi\left(Y\left(a_{2}, w_{2}\right) a_{1}\right)^{*}=\left(w_{2}+1\right)^{-\operatorname{deg} a_{1}-\operatorname{deg} a_{2}} \pi\left(Y\left(a_{1}, w_{1}\right) a_{2}, \frac{-w_{2}}{w_{2}+1}\right)^{*},
$$


and by (2.1.3), we have

$$
\pi\left(a_{2}\right)^{*} \pi\left(a_{1}\right)^{*}-\pi\left(a_{1}\right)^{*} \pi\left(a_{2}\right)^{*}=\operatorname{Res}_{w}\left(Y\left(a_{1}, w\right) a_{2}(w+1)^{\operatorname{deg} a_{1}-1}\right)^{*} .
$$

Applying (2.2.13) and (2.2.14), it is easy to prove that the sum of the first four terms on the right hand side of (2.2.12) vanishes. Using the identities (they are direct corollaries of the Jacobi identity 1.2.7)

$$
\operatorname{Res}_{w_{2}} \operatorname{Res}_{w_{1}-w_{2}}\left(\frac{z_{1}^{-\operatorname{deg} a_{1}} z_{2}^{-\operatorname{deg} a_{2}+1}\left(z_{k}+w_{1}\right)^{\operatorname{deg} a_{1}}\left(z_{k}+w_{2}\right)^{\operatorname{deg} a_{2}}}{\left(z_{1}\left(z_{k}+w_{2}\right)-z_{2}\left(z_{k}+w_{1}\right)\right)\left(z_{2}-z_{k}-w_{2}\right)}\right.
$$

$$
\begin{gathered}
\left.\cdot Y\left(\left(a_{1}, w_{1}-w_{2}\right) a_{2}, w_{2}\right)\right) \\
=\operatorname{Res}_{w_{1}} \operatorname{Res}_{w_{2}}\left(\frac{z_{1}^{-\operatorname{deg} a_{1}} z_{2}^{-\operatorname{deg} a_{2}+1}\left(z_{k}+w_{1}\right)^{\operatorname{deg} a_{1}}\left(z_{k}+w_{2}\right)^{\operatorname{deg} a_{2}}}{\left(z_{1}\left(z_{k}+w_{2}\right)-z_{2}\left(z_{k}+w_{1}\right)\right)\left(z_{2}-z_{k}-w_{2}\right)}\right. \\
\left.\cdot Y\left(a_{1}, w_{1}\right) Y\left(a_{2}, w_{2}\right)\right) \\
-\operatorname{Res}_{w_{2}} \operatorname{Res}_{w_{1}}\left(\frac{z_{1}^{-\operatorname{deg} a_{1}} z_{2}^{-\operatorname{deg} a_{2}+1}\left(z_{k}+w_{1}\right)^{\operatorname{deg} a_{1}}\left(z_{k}+w_{2}\right)^{\operatorname{deg} a_{2}}}{\left(z_{1}\left(z_{k}+w_{2}\right)-z_{2}\left(z_{k}+w_{1}\right)\right)\left(z_{2}-z_{k}-w_{2}\right)}\right. \\
\left.\cdot Y\left(a_{2}, w_{2}\right) Y\left(a_{1}, w_{1}\right)\right),
\end{gathered}
$$

$$
\operatorname{Res}_{w_{1}} \operatorname{Res}_{w_{2}-w_{1}}\left(\frac{z_{1}^{-\operatorname{deg} a_{1}+1} z_{2}^{-\operatorname{deg} a_{2}}\left(z_{k}+w_{1}\right)^{\operatorname{deg} a_{1}}\left(z_{k}+w_{2}\right)^{\operatorname{deg} a_{2}}}{\left(z_{2}\left(z_{k}+w_{1}\right)-z_{1}\left(z_{k}+w_{2}\right)\right)\left(z_{1}-z_{k}-w_{1}\right)}\right.
$$

$$
\begin{gathered}
\left.\cdot Y\left(\left(a_{2}, w_{2}-w_{1}\right) a_{1}, w_{1}\right)\right) \\
=\operatorname{Res}_{w_{2}} \operatorname{Res}_{w_{1}}\left(\frac{z_{1}^{-\operatorname{deg} a_{1}+1} z_{2}^{-\operatorname{deg} a_{2}}\left(z_{k}+w_{1}\right)^{\operatorname{deg} a_{1}}\left(z_{k}+w_{2}\right)^{\operatorname{deg} a_{2}}}{\left(z_{2}\left(z_{k}+w_{1}\right)-z_{1}\left(z_{k}+w_{2}\right)\right)\left(z_{1}-z_{k}-w_{1}\right)}\right. \\
\left.\cdot Y\left(a_{2}, w_{2}\right) Y\left(a_{1}, w_{1}\right)\right) \\
-\operatorname{Res}_{w_{1}} \operatorname{Res}_{w_{2}}\left(\frac{z_{1}^{-\operatorname{deg} a_{1}+1} z_{2}^{-\operatorname{deg} a_{2}}\left(z_{k}+w_{1}\right)^{\operatorname{deg} a_{1}}\left(z_{k}+w_{2}\right)^{\operatorname{deg} a_{2}}}{\left(z_{2}\left(z_{k}+w_{1}\right)-z_{1}\left(z_{k}+w_{2}\right)\right)\left(z_{1}-z_{k}-w_{1}\right)}\right. \\
\left.\cdot Y\left(a_{1}, w_{1}\right) Y\left(a_{2}, w_{2}\right)\right),
\end{gathered}
$$

it is easy to prove that the sum of the last four terms of the right hand side of (2.2.12) is also zero. So we have proved (2.2.11). In particular (2.2.7) holds for the 
case $n=2$. Using induction on $n$, we assume (2.2.7) is valid for $n-1$. By the recurrent definition (2.2.6) and the induction assumption, we have

$$
\begin{aligned}
& S\left(v^{\prime},\left(a_{1}, z_{1}\right)\left(a_{2}, z_{2}\right) \ldots\left(a_{n}, z_{n}\right) v\right) \\
& =S\left(v^{\prime},\left(a_{1}, z_{1}\right)\left(a_{i_{2}}, z_{i_{2}}\right) \ldots\left(a_{i_{n}}, z_{i_{n}}\right) v\right) .
\end{aligned}
$$

for every permutation $\left\{i_{2}, \ldots, i_{n}\right\}$ of $\{2, \ldots, n\}$. This together with (2.2.11) implies that (2.2.7) holds for $n$. This completes the proof of (2.2.7) for every $n$.

We now prove (2.2.8). Using the identity

$$
\frac{d}{d z} F_{n, i}(z, w)=(-i-1) F_{n+1, i+1}(z, w)
$$

which can easily be proved using generating function techniques, we have

$$
\begin{aligned}
S & \left(v^{\prime},\left(L_{-1} a_{1}, z_{1}\right)\left(a_{2}, z_{2}\right) \ldots\left(a_{n}, z_{n}\right) v\right) \\
= & \sum_{k=2}^{n} \sum_{i=0}^{\infty} F_{\operatorname{deg} a_{1}+1, i}\left(z_{1}, z_{k}\right) S\left(v^{\prime},\left(a_{2}, z_{2}\right) \ldots\left(\left(L_{-1} a_{1}\right)(i) a_{k}, z_{k}\right) \ldots\left(a_{n}, z_{n}\right) v\right) \\
& \quad+z_{1}^{-\operatorname{deg} a_{1}-1} S\left(\pi\left(L_{-1} a_{1}\right)^{*} v^{\prime},\left(a_{2}, z_{2}\right) \ldots\left(a_{n}, z_{n}\right) v\right) \\
= & \sum_{k=2}^{n} \sum_{i=0}^{\infty}(-i) F_{\operatorname{deg} a_{1}+1, i}\left(z_{1}, z_{k}\right) S\left(v^{\prime},\left(a_{2}, z_{2}\right) \ldots\left(a_{1}(i-1) a_{k}, z_{k}\right) \ldots\left(a_{n}, z_{n}\right) v\right) \\
& \quad+\left(-\operatorname{deg} a_{1}\right) z_{1}^{-\operatorname{deg} a_{1}-1} S\left(\pi\left(a_{1}\right)^{*} v^{\prime},\left(a_{2}, z_{2}\right) \ldots\left(a_{n}, z_{n}\right) v\right) \\
= & \frac{d}{d z_{1}} S\left(v^{\prime},\left(a_{1}, z_{1}\right)\left(a_{2}, z_{2}\right) \ldots\left(a_{n}, z_{n}\right) v\right) .
\end{aligned}
$$

This proves (2.2.8).

Concerning (2.2.9), for the case $k \geq 0$, it can be proved directly by using the definition (2.2.6). For the case $k<0$, we first prove that $(2.2 .9)$ holds for $k=-1$. We use induction on $n$. If $n=2$,

$$
\begin{aligned}
& \int_{C} S\left(v^{\prime},\left(a_{1}, z_{1}\right)\left(a_{2}, z_{2}\right) v\right)\left(z_{1}-z_{2}\right)^{-1} d z_{1} \\
& =\int_{C} z_{1}^{-\operatorname{deg} a_{1}} z_{2}^{-\operatorname{deg} a_{2}}\left(\pi\left(a_{2}\right)^{*} \pi\left(a_{1}\right)^{*} v^{\prime}, v\right)\left(z_{1}-z_{2}\right)^{-1} d z_{1} \\
& +\sum_{i=0}^{\infty} \int_{C} F_{\operatorname{deg} a_{1}, i}\left(z_{1}, z_{2}\right) z_{2}^{-\operatorname{deg} a_{1}-\operatorname{deg} a_{2}+i+1}\left(\pi\left(\left(a_{1}\right)(i) a_{2}\right)^{*} v^{\prime}, v\right)\left(z_{1}-z_{2}\right)^{-1} d z_{1} \\
& =z_{2}^{-\operatorname{deg} a_{1}-\operatorname{deg} a_{2}}\left(\pi\left(a_{2}\right)^{*} \pi\left(a_{1}\right)^{*} v^{\prime}, v\right)-z_{2}^{-\operatorname{deg} a_{1}-\operatorname{deg} a_{2}} \sum_{i=0}^{\infty}\left(\begin{array}{c}
\operatorname{deg} a_{1} \\
i+1
\end{array}\right)\left(\pi\left(a_{1}(i) a_{2}\right)^{*} v^{\prime}, v\right) \\
& \left.=z_{2}^{-\operatorname{deg} a_{1}-\operatorname{deg} a_{2}} \pi\left(a_{1}(-1) a_{2}\right)^{*} v^{\prime}, v\right) \\
& =S\left(v^{\prime},\left(a_{1}(-1) a_{2}, z_{2}\right) v\right) \text {. }
\end{aligned}
$$

Note that we have used $\pi\left(a_{1} * a_{2}\right)=\pi\left(a_{1}\right) \pi\left(a_{2}\right)$ in the above computation. Suppose that for the case $n-1$ we have

$$
\begin{aligned}
& \int_{C} S\left(v^{\prime},\left(a_{1}, z_{1}\right)\left(a_{2}, z_{2}\right) \ldots\left(a_{n-1}, z_{n-1}\right) v\right)\left(z_{1}-z_{2}\right)^{-1} d z_{1} \\
& =S\left(v^{\prime},\left(a_{1}(-1) a_{2}, z_{2}\right) \ldots\left(a_{n-1}, z_{n-1}\right) v\right) .
\end{aligned}
$$


We need to prove

$$
\begin{aligned}
& \int_{C} S\left(v^{\prime},\left(a_{1}, z_{1}\right)\left(a_{2}, z_{2}\right) \ldots\left(a_{n}, z_{n}\right) v\right)\left(z_{1}-z_{2}\right)^{-1} d z_{1} \\
& =S\left(v^{\prime},\left(a_{1}(-1) a_{2}, z_{2}\right) \ldots\left(a_{n}, z_{n}\right) v\right),
\end{aligned}
$$

By (2.2.7), it is equivalent to

$$
\begin{aligned}
& \int_{C} S\left(v^{\prime},\left(a_{1}, z_{1}\right)\left(a_{2}, z_{2}\right)\left(a_{3}, z_{3}\right) \ldots\left(a_{n}, z_{n}\right) v\right)\left(z_{2}-z_{3}\right)^{-1} d z_{2} \\
& =S\left(v^{\prime},\left(a_{1}, z_{1}\right)\left(a_{2}(-1) a_{3}, z_{3}\right) \ldots\left(a_{n}, z_{n}\right) v\right)
\end{aligned}
$$

Using the induction assumption (2.2.19), and using the formula

$$
F_{\operatorname{deg} a_{1}, i}\left(z_{1}, z_{2}\right)\left(z_{2}-z_{3}\right)^{-1}=\sum_{j=0}^{\infty}\left(\begin{array}{c}
i+j \\
i
\end{array}\right) F_{\operatorname{deg} a_{1}, i+j}\left(z_{1}, z_{3}\right)\left(z_{2}-z_{3}\right)^{j-1},
$$

which can easily be proved using generating function techniques, we have

$$
\begin{aligned}
& \int_{C} S\left(v^{\prime},\left(a_{1}, z_{1}\right)\left(a_{2}, z_{2}\right)\left(a_{3}, z_{3}\right) \ldots\left(a_{n}, z_{n}\right) v\right)\left(z_{2}-z_{3}\right)^{-1} d z_{2} \\
& =\int_{C} z_{1}^{-\operatorname{deg} a_{1}} S\left(\pi\left(a_{1}\right)^{*} v^{\prime},\left(a_{2}, z_{2}\right)\left(a_{3}, z_{3}\right) \ldots\left(a_{n}, z_{n}\right) v\right) . \\
& \quad+\left(z_{2}-z_{3}\right)^{-1} d z_{2} \\
& \quad+\int_{C} \sum_{i \in \mathbf{N}} F_{\operatorname{deg} a_{1}, i}\left(z_{1}, z_{2}\right) S\left(v^{\prime},\left(a_{1}(i) a_{2}, z_{2}\right)\left(a_{3}, z_{3}\right) \ldots\left(a_{n}, z_{n}\right) v\right)\left(z_{2}-z_{3}\right)^{-1} d z_{2} \\
& \quad+\sum_{k=3}^{n} \int_{C} \sum_{i \in \mathbf{N}} F_{\operatorname{deg} a_{1}, i}\left(z_{1}, z_{k}\right) \\
& \left.\quad \cdot z_{1}^{-\operatorname{deg} a_{1}} S\left(\pi\left(a^{\prime},\left(a_{2}, z_{2}\right)\left(a_{3}, z_{3}\right) \ldots\left(a_{1}(i) a_{k}, z_{k}\right) \ldots\left(a_{n}, z_{n}\right) v\right)\left(z_{2}-z_{3}\right)^{-1} d a_{2}, z_{3}\right) \ldots\left(a_{n}, z_{n}\right) v\right) \\
& \quad+\sum_{i \in \mathbf{N}} \sum_{j \in \mathbf{N}}\left(\begin{array}{c}
i+j \\
i
\end{array}\right) F_{\operatorname{deg} a_{1}, i+j}\left(z_{1}, z_{3}\right) S\left(v^{\prime},\left(\left(a_{1}(i) a_{2}\right)(j-1) a_{3}, z_{3}\right) \ldots\left(a_{n}, z_{n}\right) v\right) \\
& \quad+\sum_{k=3}^{n} \sum_{i \in \mathbf{N}} F_{\operatorname{deg} a_{1}, i}\left(z_{1}, z_{k}\right) S\left(v^{\prime},\left(a_{2}(-1) a_{3}, z_{3}\right) \ldots\left(a_{1}(i) a_{k}, z_{k}\right) \ldots\left(a_{n}, z_{n}\right) v\right) .
\end{aligned}
$$

On the other hand, we have

$$
\begin{aligned}
& S\left(v^{\prime},\left(a_{1}, z_{1}\right)\left(a_{2}(-1) a_{3}, z_{3}\right) \ldots\left(a_{n}, z_{n}\right) v\right) \\
& =z_{1}^{-\operatorname{deg} a_{1}} S\left(\pi\left(a_{1}\right)^{*} v^{\prime},\left(a_{2}(-1) a_{3}, z_{3}\right) \ldots\left(a_{n}, z_{n}\right) v\right) \\
& \quad+\sum_{i \in \mathbf{N}} F_{\operatorname{deg} a_{1}, i}\left(z_{1}, z_{3}\right) S\left(v^{\prime},\left(a_{1}(i) a_{2}(-1) a_{3}, z_{3}\right) \ldots\left(a_{n}, z_{n}\right) v\right) \\
& \quad+\sum_{k=4}^{n} \sum_{i \in \mathbf{N}} F_{\operatorname{deg} a_{1}, i}\left(z_{1}, z_{k}\right) S\left(v^{\prime},\left(a_{2}(-1) a_{3}, z_{3}\right) \ldots\left(a_{1}(i) a_{k}, z_{k}\right) \ldots\left(a_{n}, z_{n}\right) v\right) .
\end{aligned}
$$


Comparing (2.2.22) and (2.2.23), we see that (2.2.21) reduces to the identity

$$
\sum_{i=0}^{m}\left(\begin{array}{c}
m \\
i
\end{array}\right)\left(a_{1}(i) a_{2}\right)(m-i-1)+a_{2}(-1) a_{1}(m)=a_{1}(m) a_{2}(-1) \quad \text { for } m \geq 0,
$$

which is a direct corollary of (1.2.11). So we have proved (2.2.20) for arbitrary $n \geq 2$. Now we use induction on $k$ to prove

$$
\begin{aligned}
& \int_{C} S\left(v^{\prime},\left(a_{1}, z_{1}\right)\left(a_{2}, z_{2}\right) \ldots\left(a_{n}, z_{n}\right) v\right)\left(z_{1}-z_{2}\right)^{-k} d z_{1} \\
& =S\left(v^{\prime},\left(a_{1}(-k) a_{2}, z_{2}\right) \ldots\left(a_{n}, z_{n}\right) v\right) .
\end{aligned}
$$

The case $k=1$ is just (2.2.20). Suppose (2.2.24) holds for $k$. Then for $k+1$,

$$
\begin{aligned}
& \int_{C} S\left(v^{\prime},\left(a_{1}, z_{1}\right)\left(a_{2}, z_{2}\right) \ldots\left(a_{n}, z_{n}\right) v\right)\left(z_{1}-z_{2}\right)^{-k-1} d z_{1} \\
& =\frac{1}{-k} \int_{C} S\left(v^{\prime},\left(a_{1}, z_{1}\right)\left(a_{2}, z_{2}\right) \ldots\left(a_{n}, z_{n}\right) v\right) \frac{d}{d z_{1}}\left(z_{1}-z_{2}\right)^{-k} d z_{1} \\
& =\frac{1}{k} \int_{C} \frac{d}{d z_{1}} S\left(v^{\prime},\left(a_{1}, z_{1}\right)\left(a_{2}, z_{2}\right) \ldots\left(a_{n}, z_{n}\right) v\right)\left(z_{1}-z_{2}\right)^{-k} d z_{1} \\
& =\frac{1}{k} \int_{C} S\left(v^{\prime},\left(L_{-1} a_{1}, z_{1}\right)\left(a_{2}, z_{2}\right) \ldots\left(a_{n}, z_{n}\right) v\right)\left(z_{1}-z_{2}\right)^{-k} d z_{1} \\
& =\frac{1}{k} S\left(v^{\prime},\left(\left(L_{-1} a_{1}\right)(-k) a_{2}, z_{2}\right) \ldots\left(a_{n}, z_{n}\right) v\right) \\
& =S\left(v^{\prime},\left(a_{1}(-k-1) a_{2}, z_{2}\right) \ldots\left(a_{n}, z_{n}\right) v\right) .
\end{aligned}
$$

This completes the proof of (2.2.9).

Step 3 . Let $\bar{M}$ be the vector space spanned by the symbols

$$
\left(b_{1}, i_{1}\right)\left(b_{2}, i_{2}\right) \ldots\left(b_{m}, i_{m}\right) v
$$

for $b_{k} \in V, i_{k} \in \mathbf{Z}, v \in W$, and (2.2.25) linear for each $b_{i}$. We define a gradation on $\bar{M}$ :

$$
\operatorname{deg}\left(\left(b_{1}, i_{1}\right)\left(b_{2}, i_{2}\right) \ldots\left(b_{m}, i_{m}\right)\right)=\sum_{k=1}^{m}\left(\operatorname{deg} b_{k}-1-i_{k}\right),
$$

so we have $\bar{M}=\bigoplus_{n=-\infty}^{\infty} \bar{M}_{n}$. Note that $W$ is a subspace of $\bar{M}_{0}$.

Recall that in Step 1 we actually defined a linear map

$$
S: \quad W^{*} \otimes V \otimes \cdots \otimes V \otimes W \rightarrow \text { rational functions. }
$$

Now we extend $S$ to $W^{*} \otimes V \otimes \cdots \otimes V \otimes \bar{M}$ as follows. For $m$ as in (2.2.25), we define

$(2.2 .26)$

$S\left(v^{\prime},\left(a_{1}, z_{1}\right) \ldots\left(a_{n}, z_{n}\right) m\right)$

$$
=\int_{C_{1}} \ldots \int_{C_{m}} S\left(v^{\prime},\left(a_{1}, z_{1}\right) \ldots\left(a_{n}, z_{n}\right)\left(b_{1}, w_{1}\right) \ldots\left(b_{m}, w_{m}\right) v\right) w_{1}^{i_{1}} \ldots w_{m}^{i_{m}} d w_{1} \ldots d w_{m},
$$


where $C_{k}$ is a contour for $w_{k}, z_{i}(i=1,2, \ldots, n)$ are outside each $C_{k}, C_{k}$ contains $C_{k+1}$ and $C_{m}$ contains 0 . It is clear that $S\left(v^{\prime},\left(a_{1}, z_{1}\right)\left(a_{2}, z_{2}\right) \ldots\left(a_{n}, z_{n}\right) m\right)$ possess properties similar to those of (2.2.7)-(2.2.9), namely

$$
\begin{aligned}
& S\left(v^{\prime},\left(a_{1}, z_{1}\right)\left(a_{2}, z_{2}\right) \ldots\left(a_{n}, z_{n}\right) m\right) \\
& =S\left(v^{\prime},\left(a_{i_{1}}, z_{i_{1}}\right)\left(a_{i_{2}}, z_{i_{2}}\right) \ldots\left(a_{i_{n}}, z_{i_{n}}\right) m\right), \\
& S\left(v^{\prime},\left(L_{-1} a_{1}, z_{1}\right)\left(a_{2}, z_{2}\right) \ldots\left(a_{n}, z_{n}\right) m\right) \\
& =\frac{d}{d z_{1}} S\left(v^{\prime},\left(a_{1}, z_{1}\right)\left(a_{2}, z_{2}\right) \ldots\left(a_{n}, z_{n}\right) m\right), \\
& \int_{C} S\left(v^{\prime},\left(a_{1}, z_{1}\right)\left(a_{2}, z_{2}\right) \ldots\left(a_{n}, z_{n}\right) m\right)\left(z_{1}-z_{2}\right)^{k} d z_{1} \\
& =S\left(v^{\prime},\left(a_{1}(k) a_{2}, z_{2}\right)\left(a_{3}, z_{3}\right) \ldots\left(a_{n}, z_{n}\right) m\right) .
\end{aligned}
$$

where $\left\{i_{1}, i_{2}, \ldots, i_{n}\right\}$ is an arbitrary permutation of $\{1,2, \ldots, n\}, k$ is an arbitrary integer, and $C$ is a contour of $z_{1}$ surrounding $z_{2}$.

Step 4. Define

$\operatorname{Rad}(\bar{M})=\left\{m \mid S\left(v^{\prime},\left(a_{1}, z_{1}\right) \ldots\left(a_{n}, z_{n}\right) m\right)=0\right.$ for every $\left.n \geq 0, a_{i} \in V, v^{\prime} \in W^{*}\right\}$.

The quotient space $\bar{M} / \operatorname{Rad}(\bar{M})$ will be our desired $V$-module. It is clear that $\operatorname{Rad}(\bar{M})$ is a direct sum of homogeneous subspaces, so $\bar{M} / \operatorname{Rad}(\bar{M})$ has a reduced gradation. And it is easy to see from the definition that

$$
\begin{gathered}
\text { if } m \in \operatorname{Rad}(\bar{M}), \text { then }(a, i) m \in \operatorname{Rad}(\bar{M}), \\
W \cap \operatorname{Rad}(\bar{M})=\{0\} .
\end{gathered}
$$

By induction on $m$ and by counting the orders of poles of $w_{1}, \ldots, w_{m}$ in the functions

$$
S\left(v^{\prime},\left(a_{1}, z_{1}\right) \ldots\left(a_{n}, z_{n}\right)\left(b_{1}, w_{1}\right) \ldots\left(b_{m}, w_{m}\right) v\right) w_{1}^{i_{1}} \ldots w_{m}^{i_{m}},
$$

it can be proved that if $\sum_{k=1}^{m}\left(\operatorname{deg} b_{k}-1-i_{k}\right)<0$ and $v \in W$, then

$$
\left(b_{1}, i_{1}\right) \cdots\left(b_{m}, i_{m}\right) v \in \operatorname{Rad}(\bar{M}) .
$$

So we have

$$
\bigoplus_{n=-1}^{-\infty} \bar{M}(n) \subset \operatorname{Rad}(\bar{M})
$$

We claim that the quotient space $M=\bar{M} / \operatorname{Rad}(\bar{M})=\oplus_{n=0}^{\infty} M_{n}$ is a representation of $V$ with the action of the vertex operator $Y(a, z)$ on $M$ as follows: for

$$
\left(b_{1}, i_{1}\right)\left(b_{2}, i_{2}\right) \ldots\left(b_{m}, i_{m}\right) v \in M \quad(v \in W),
$$

$$
Y_{M}(a, z)\left(b_{1}, i_{1}\right)\left(b_{2}, i_{2}\right) \ldots\left(b_{m}, i_{m}\right) v=(a, n)\left(b_{1}, i_{1}\right)\left(b_{2}, i_{2}\right) \ldots\left(b_{m}, i_{m}\right) v z^{-n-1},
$$

and $M$ satisfies the conditions in our theorem. 
The rest of this step is to prove the above claim.

We first prove $\bar{M}$ is a representation of $V$. It is clear that (1.2.18), (1.2.19) and (1.2.24) in the definition of a representation hold. We next sketch a proof of the Jacobi identity (1.2.20). By the definition of $\operatorname{Rad}(\bar{M})$, we know that linear maps $S$ from the spaces $W^{*} \otimes V \otimes \cdots \otimes V \rightarrow \bar{M}$ to the space of rational functions reduce to linear maps $S$ from $W^{*} \otimes V \otimes \cdots \otimes V \rightarrow M$ to the space of rational functions, and these maps satisfy $(2.2 .27),(2.2 .28)$ and (2.2.29). And for $v^{\prime} \in W^{*}, a_{i} \in V$ and $m \in M$,

$$
S\left(v^{\prime},\left(a_{1}, z_{1}\right) \ldots\left(a_{n}, z_{n}\right) m\right)
$$

has Laurent series expansion on the domain $\left|z_{1}\right|>\cdots>\left|z_{n}\right|>0$

$$
\sum_{i_{1} \in \mathbf{Z}} \cdots \sum_{i_{n} \in \mathbf{Z}} S\left(v^{\prime}, a_{1}\left(i_{1}\right) \ldots a_{n}\left(i_{n}\right) m\right) z_{1}^{-i_{1}-1} \ldots z_{n}^{-i_{n}-1}
$$

this follows from the identity

$$
\begin{aligned}
& \int_{C_{1}} \ldots \int_{C_{n}} S\left(v^{\prime},\left(a_{1}, z_{1}\right) \ldots\left(a_{n}, z_{n}\right) v\right) z_{1}^{i_{1}} \ldots z_{n}^{i_{n}} d z_{1} \ldots d z_{n} \\
& =S\left(v^{\prime}, a_{1}\left(i_{1}\right) \cdots a_{n}\left(i_{n}\right) m\right)
\end{aligned}
$$

where the contour $C_{k}$ is for $z_{k}, C_{k}$ contains $C_{k+1}$, and $C_{m}$ contains 0 . Using this expansion property of rational functions $S$, together with $(2.2 .27),(2.2 .29)$ and the Cauchy Theorem for contour integrals, we can prove the Jacobi identity (1.2.20). To prove $M$ is a representation, it remains to prove the Virasoro axiom (1.2.21) and (1.2.23). (1.2.23) follows directly from (2.2.28). The truth of the Jacobi identity implies in particular the truth of (1.2.11). Using (1.2.11) and the fact that $L_{0} \omega=$ $2 \omega, L_{1} \omega=0$ and $L_{2} \omega=1 / 2 c$ (these formulas are corollaries of (1.2.10) and the Viraroso relations on $V$ ), it is easy to prove (1.2.21). This completes the proof of the fact that $M$ is a representation.

(2.2.32) implies that the natural map $W \rightarrow M_{0}$ is injective. To prove that this map is also surjective, we need to prove that for $v \in W$,

$$
b(\operatorname{deg} b-1) v=\pi(b) v
$$

Using the commutation relation $(1.2 .11)$ and the identity $S\left(v^{\prime}, a(\operatorname{deg} a-1) m\right)=$ $S\left(\pi(a)^{*} v^{\prime}, m\right)\left(v^{\prime} \in W^{*}, a \in V\right.$ and $\left.m \in M\right)$, it can be proved that if $m \in M_{0}$ satisfies $S\left(v^{\prime}, m\right)=0$ for every $v^{\prime} \in W^{*}$, then $S\left(v^{\prime}, a_{1}\left(i_{1}\right) \cdots a_{n}\left(i_{n}\right) m\right)=0$ for every $n, a_{k} \in V$ and $i_{k} \in \mathbf{Z}$, which further implies that $m=0$. With the above fact in mind, (2.2.35) follows from

$$
S\left(v^{\prime}, b(\operatorname{deg} b-1) v\right)=S\left(v^{\prime}, \pi(b) v\right)
$$

from every $v^{\prime} \in W^{*}$. So we have proved the the top level of $M$ is isomorphic to $W$ as an $A(V)$-module.

Clearly, the rational function $S\left(v^{\prime},\left(a_{1}, z_{1}\right) \ldots\left(a_{n}, z_{n}\right) v\right)\left(v^{\prime} \in W^{*}, a_{i} \in V\right.$ and $v \in W)$ is the correlation function $\left(v^{\prime}, Y\left(a_{1}, z_{1}\right) \cdots Y\left(a_{n}, z_{n}\right) v\right)$. From this it is easy to see that a subrepresentation $N=\bigoplus_{n=0}^{\infty} N_{n}$ satisfying $N_{0}=\{0\}$ is necessarily $\{0\}$. This completes the proof of the theorem. 
From the theorem and its proof, we know that if an $A(V)$-module $W$ is irreducible, then the rerepresentation $M$ constructed in the proof is also irreducible. Conversely, it is easy to see that if $M=\bigoplus_{n=0}^{\infty} M_{n}$ is an irreducible representation, then $M_{0}$ is an irreducible $A(V)$-module. And from the above proof, we can prove that two irreducible $V$-modules with top levels isomorphic as $A(V)$-modules are isomorphic. So we have the following theorem.

Theorem 2.2.2. The isomorphism classes of the irreducible representations of $V$ and the isomorphism classes of the irreducible representations of $A(V)$ are in oneto-one correspondence.

Theorem 2.2.3. If $V$ is rational, then $A(V)$ is a semi-simple associative algebra.

Proof. We view $A(V)$ as an $A(V)$-module by left multiplication. Using Theorem 2.2 .1 , there exists a $V$-module $M$ such the top level of $M$ is $A(V)$. It is clear that $M$ is finitely generated. The rationality of $V$ implies that $M$ is a finite direct sum of finitely many irreducible modules, and so the top level is a direct sum of finitely many irreducible $A(V)$-modules. This proves that $A(V)$ is finite dimensional and semisimple.

\section{3. $q$-EXPANSIONS OF MODULAR FORMS AND ELLIPTIC FUNCTIONS}

This section collects some facts about the Eisenstein series and the Weierstrass $\wp$-functions. Their $q$-expansions will be needed in the computations of $q$-traces of vertex operators. Most of the formulas listed below may be found in [La] or [Ap].

The Eisenstein series $G_{2 k}(\tau)(k=1,2,3, \ldots)$ are series

$$
G_{2 k}(\tau)=\sum_{(m, n) \neq(0,0)} \frac{1}{(m \tau+n)^{2 k}} \quad \text { for } \quad k \geq 2,
$$

and, for $k=1$,

$$
G_{2}(\tau)=\frac{\pi^{2}}{3}+\sum_{m \in \mathbf{Z}-\{0\}} \sum_{n \in \mathbf{Z}} \frac{1}{(m \tau+n)^{2}} .
$$

They have the $q-$ expansions

$$
G_{2 k}(\tau)=2 \xi(2 k)+\frac{2(2 \pi i)^{2 k}}{(2 k-1) !} \sum_{n=1}^{\infty} \sigma_{2 k-1}(n) q^{n}
$$

(cf. [Se] p.44, p.249; [Ap] p.69), where $\xi(2 k)=\sum_{n=1}^{\infty} \frac{1}{n^{2 k}}$ (so $\left.\xi(2)=\frac{\pi^{2}}{6}\right), q=e^{2 \pi i \tau}$ and $\sigma_{k}(n)$ is the sum of the $k$-th powers of the divisors of $n$. Formula (3.3) can be written in the form

$$
G_{2 k}(\tau)=2 \zeta(2 k)+\frac{2(2 \pi i)^{2 k}}{(2 k-1) !} \sum_{n=1}^{\infty} \frac{n^{2 k-1} q^{n}}{1-q^{n}} .
$$

For $k \geq 2, G_{2 k}(\tau)$ is a modular form of weight $2 k$ for the modular group $S L_{2}(\mathbf{Z})$. And it is well-known that $G_{2}(\tau)$ transforms in the following way under the action of $S L_{2}(\mathbf{Z})$ :

$$
G_{2}\left(\frac{a \tau+b}{c \tau+d}\right)=(c \tau+d)^{2} G_{2}(\tau)-2 \pi i c(c \tau+d)
$$

(cf. [Ap] p.69 (50)). 
We define the functions $\wp_{k}(z, \tau)(k \geq 1)$ by

$$
\begin{aligned}
& \wp_{1}(z, \tau)=\frac{1}{z}+\sum_{\omega \in L^{\prime}}\left(\frac{1}{z-\omega}+\frac{1}{\omega}+\frac{z}{\omega^{2}}\right), \\
& \wp_{2}(z, \tau)=\frac{1}{z^{2}}+\sum_{\omega \in L^{\prime}}\left(\frac{1}{(z-\omega)^{2}}-\frac{1}{\omega^{2}}\right),
\end{aligned}
$$

and for $n \geq 2, \wp_{k+1}(z, \tau)=-\frac{1}{k} \frac{d}{d z} \wp_{k}(z, \tau)$, where $L$ denotes the lattce $\{m \tau+n\}$ and $L^{\prime}$ denotes the set of nonzero vectors in $L$.

$\wp_{2}(z, \tau)$ is usually called the Weierstrass $\wp$-function and denoted by $\wp(z, \tau)$ (cf. [La] p.7). $\wp_{k}(z, \tau)(k=1,2, \cdots)$ have the Laurent expansion near $z=0$,

$$
\wp_{k}(z, \tau)=\frac{1}{z^{k}}+(-1)^{k} \sum_{n=1}^{\infty}\left(\begin{array}{c}
2 n+1 \\
k-1
\end{array}\right) G_{2 n+2}(\tau) z^{2 n+2-k}
$$

(cf. [La] p.10, p.248). $\wp_{1}(z, \tau)$ and $\wp_{2}(z, \tau)$ have $q$-expansions

$$
\begin{aligned}
& \wp_{1}(z, \tau)=G_{2}(\tau) z+\pi i \frac{q_{z}+1}{q_{z}-1}+2 \pi i \sum_{n=1}^{\infty}\left(\frac{q_{\tau}^{n}}{q_{z}-q_{\tau}^{n}}-\frac{q_{z} q_{\tau}^{n}}{1-q_{z} q_{\tau}^{n}}\right), \\
& \wp_{2}(z, \tau)=-\frac{\pi^{2}}{3}+(2 \pi i)^{2} \sum_{m \in \mathbf{Z}} \frac{q_{\tau}^{m} q_{z}}{\left(1-q_{\tau}^{m} q_{z}\right)^{2}}-2(2 \pi i)^{2} \sum_{n=1}^{\infty} \frac{n q_{\tau}^{n}}{1-q_{\tau}^{n}},
\end{aligned}
$$

where $q_{z}=e^{2 \pi i z}, q_{\tau}=e^{2 \pi i \tau}$; the fractions in (3.7) and (3.8) are expanded in non-negative powers of $q_{\tau}$. See [La] p.248 for (3.7) and [La] p.46 for [3.8]. The $q$-expansion for $\wp_{k}(z, \tau)(k>2)$ can be obtained by the relation $\frac{d}{d z} \wp_{n}(z, \tau)=$ $-n \wp_{n+1}(z, \tau)$.

$\wp_{k}(z, \tau)$ is an elliptic function with periods 1 and $\tau$ with the exception of $k=1$. $\wp_{1}(z, \tau)$ has the transformation properties

$$
\begin{aligned}
& \wp_{1}(z+1, \tau)=\wp_{1}(z, \tau)+\eta(\tau, 1), \\
& \wp_{1}(z+\tau, \tau)=\wp_{1}(z, \tau)+\eta(\tau, \tau),
\end{aligned}
$$

where $\eta(\tau, 1)=G_{2}(\tau)$ and $\eta(\tau, \tau)$ is determined by the equation

$$
\eta(\tau, 1) \tau-\eta(\tau, \tau)=2 \pi i
$$

which is called the Legendre relation, see [La] p.240-241.

Since our computations of the $q$-trace of vertex operators are formal, we denote by $\widetilde{G}_{2 k}(q)$ the $q$-expansion of the Eisenstein series $G_{2 k}(\tau)$, so $\widetilde{G}_{2 k}(q) \in \mathbf{C}[[q]]$, and

$$
\widetilde{G}_{2 k}(q)=2 \xi(2 k)+\frac{2(2 \pi i)^{2 k}}{(2 k-1) !} \sum_{n=1}^{\infty} \sigma_{2 k-1}(n) q^{n} .
$$

We set $\widetilde{G}_{k}(q)=0$ for odd $k$. 
We will frequently meet the the formal power series

$$
P_{k}(z, q)=\frac{(2 \pi i)^{k}}{(k-1) !} \sum_{n=1}^{\infty}\left(\frac{n^{k-1} z^{n}}{1-q^{n}}+\frac{(-1)^{k} n^{k-1} z^{-n} q^{n}}{1-q^{n}}\right),
$$

where $\frac{1}{1-q^{n}}$ is understood as $\sum_{i=0}^{\infty} q^{n i}$. Note that $z \frac{d}{d z} P_{k}(z, q)=\frac{k}{2 \pi i} P_{k+1}(z, q)$. It is easy to prove that the formal power series (3.9) converges uniformly and absolutely in every closed subset of the domain $\{(z, q)|| q|<| z \mid<1\}$.

The limit of $P_{k}(z, q)$ (which we still denote as $P_{k}(z, q)$ ) relates to $\wp_{k}(z, \tau)$ by the formulas:

$$
\begin{gathered}
P_{1}\left(q_{z}, q\right)=-\wp_{1}(z, \tau)+G_{2}(\tau) z-\pi i, \\
P_{2}\left(q_{z}, q\right)=\wp_{2}(z, \tau)+G_{2}(\tau),
\end{gathered}
$$

and for $k>2$

$$
P_{k}\left(q_{z}, q\right)=(-1)^{k} \wp_{k}(z, \tau)
$$

where $q_{z}=e^{2 \pi i z}$ and $q=e^{2 \pi i \tau}$. The proof of (3.11) uses (3.7) and the formula

$$
\sum_{n=1}^{\infty}\left(\frac{q^{n} / w}{1-q^{n} / w}-\frac{q^{n} w}{1-q^{n} w}\right)=\sum_{n=1}^{\infty} \frac{q^{n}}{1-q^{n}}\left(w^{-n}-w^{n}\right)
$$

(see [La] p.249). (3.12) is proved using the relation $z \frac{d}{d z} P_{1}(z, q)=\frac{1}{2 \pi i} P_{2}(z, q)$ and (3.11).

\section{Correlation functions on the torus}

In this section we first define in an abstract way the correlation functions and conformal block on the torus for a given vertex operator algebra $V$. Then we prove that for $V$ satisfying a certain finiteness condition and a $V$-module $M=\bigoplus_{k=0}^{\infty} M_{k}$ with $\operatorname{dim} M_{k}<\infty$ and $\left.L_{0}\right|_{M_{k}}=(k+h) I d$ for some constant $h$, the formal power series of variables $z_{1}, \ldots, z_{n}, q$ defined by the trace of vertex operators

$$
\left.\operatorname{tr}\right|_{M} Y\left(a_{1}, z_{1}\right) \ldots Y\left(a_{n}, z_{n}\right) q^{L_{0}}
$$

$\left(n=1,2, \ldots ; a_{i} \in V\right)$ converges on the domain $|q|<\left|z_{n}\right|<\cdots<\left|z_{1}\right|<1$, and we verify that the limits give the $n$-point correlation functions on the torus $\mathbf{C}-\{0\} / z \sim z q^{k}$, the punctured plane $\mathbf{C}-\{0\}$ modulo the relations $z=z q^{k}$ for $k \in \mathbf{Z}$.

4.1. Conformal block on the torus. We first fix some notations on multivariable doubly periodic functions. Let $H$ be the upper half complex plane, whose element will be denoted as $\tau$. For $n=1,2, \ldots$, let $\mathcal{F}_{n}$ denote the space of meromorphic functions $f\left(z_{1}, \ldots, z_{n}, \tau\right)$ on $\mathbf{C}^{n} \times H$ such that for fixed $\tau, f\left(z_{1}, \ldots, z_{n}, \tau\right)$ is doubly periodic for each variable $z_{i}$ with periods $s+t \tau(s, t \in \mathbf{Z})$ and the possible poles of $f\left(z_{1}, \ldots, z_{n}, \tau\right)$ lie in the set

$$
\left\{\left(z_{1}, \ldots, z_{n}, \tau\right) \mid z_{i}=z_{j}+(s+t \tau), i \neq j ; s, t \in \mathbf{Z}\right\} .
$$

Note that a function $f(z, \tau)$ in $\mathcal{F}_{1}$ is independent of $z$ since $f(z, \tau)$ is doubly periodic without poles, thus $\mathcal{F}_{1}$ is the space of holomorphic functions on $H$. 
Definition 4.1.1. Let $\{V, Y(, z), 1, \omega\}$ be a vertex operator algebra. A system of maps $S=\left\{S_{n}\right\}_{n=1}^{\infty}$,

$$
S_{n}: V^{n} \rightarrow \mathcal{F}_{n},\left(a_{1}, \ldots, a_{n}\right) \mapsto S\left(\left(a_{1}, z_{1}\right), \ldots,\left(a_{n}, z_{n}\right), \tau\right)
$$

is said to satisfy the genus-one property if the following properties (1)-(6) are satisfied.

(1) $S\left(\left(a_{1}, z_{1}\right),\left(a_{2}, z_{2}\right), \ldots,\left(a_{n}, z_{n}\right), \tau\right)$ is linear for each $a_{i}$.

(2) For every permutation $\sigma$ of $n$ letters,

$$
S\left(\left(a_{\sigma_{1}}, z_{\sigma_{1}}\right), \ldots,\left(a_{\sigma_{n}}, z_{\sigma_{n}}\right), \tau\right)=S\left(\left(a_{1}, z_{1}\right), \ldots,\left(a_{n}, z_{n}\right), \tau\right) .
$$

$$
S\left((1, z),\left(a_{1}, z_{1}\right), \ldots,\left(a_{n}, z_{n}\right), \tau\right)=S\left(\left(a_{1}, z_{1}\right), \ldots,\left(a_{n}, z_{n}\right), \tau\right) .
$$

$$
S\left(\left(L_{-1} a_{1}, z_{1}\right), \ldots,\left(a_{n}, z_{n}\right), \tau\right)=\frac{d}{d z_{1}} S\left(\left(a_{1}, z_{1}\right), \ldots,\left(a_{n}, z_{n}\right), \tau\right)
$$

$$
\begin{aligned}
& \int_{C} S\left((a, w),\left(a_{1}, z_{1}\right), \ldots,\left(a_{n}, z_{n}\right), \tau\right)\left(w-z_{1}\right)^{k} d w \\
& =S\left(\left(a(k) a_{1}, z_{1}\right), \ldots,\left(a_{n}, z_{n}\right), \tau\right),
\end{aligned}
$$

where $C$ is a contour of $w$ surrounding $z_{1}$.

(6) If $a_{i}(i=1, \ldots, n)$ are highest weight vectors for the Virasoro algebra with degree $\operatorname{deg} a_{i}$, write

$$
S=S\left(\left(\omega, w_{1}\right), \ldots,\left(\omega, w_{m}\right),\left(a_{1}, z_{1}\right), \ldots,\left(a_{n}, z_{n}\right), \tau\right) ;
$$

then

$$
\begin{aligned}
S & \left.(\omega, w),\left(\omega, w_{1}\right), \ldots,\left(\omega, w_{m}\right),\left(a_{1}, z_{1}\right), \ldots,\left(a_{n}, z_{n}\right), \tau\right) \\
& =2 \pi i \frac{d}{d \tau} S \\
& +\sum_{k=1}^{n}\left(\wp_{1}\left(w-z_{k}, \tau\right)-\left(w-z_{k}\right) G_{2}(\tau)\right) \frac{d}{d z_{k}} S \\
& +\sum_{k=1}^{m}\left(\wp_{1}\left(w-w_{k}, \tau\right)-\left(w-w_{k}\right) G_{2}(\tau)\right) \frac{d}{d w_{k}} S \\
& +\sum_{k=1}^{n}\left(\wp_{2}\left(w-z_{k}, \tau\right)+G_{2}(\tau)\right) \operatorname{deg} a_{k} S \\
& +\sum_{k=1}^{m}\left(\wp_{2}\left(w-w_{k}, \tau\right)+G_{2}(\tau)\right) 2 S \\
& \left.+\frac{c}{2} \sum_{k=1}^{m} \wp_{4}\left(w-z_{k}, \tau\right) S\left(\left(\omega, w_{1}\right), \ldots, \widehat{\left(\omega, w_{k}\right.}\right), \ldots,\left(a_{n}, z_{n}\right), \tau\right),
\end{aligned}
$$

where $\left(\widehat{\omega, w_{k}}\right)$ means the term is omitted. This completes the definition. 
Given such a system $\left\{S_{n}\right\}$, it is clear that for fixed $\tau, S\left(\left(a_{1}, z_{1}\right), \ldots,\left(a_{n}, z_{n}\right), \tau\right)$ can be regarded as an $n$-variable function on complex torus $\mathbf{C} /\{s \tau+t\}$. We call

$$
\left\{S\left(\left(a_{1}, z_{1}\right), \ldots,\left(a_{n}, z_{n}\right), \tau\right)\right\}
$$

$\left(n=1,2, \ldots ; a_{i} \in V\right)$ a system of correlation functions on the torus.

By the definition, it is clear that if $\left\{S_{n}^{\alpha}\right\}$ and $\left\{S_{n}^{\beta}\right\}$ satisfy the genus-one property, then so do their linear combinations $\left\{u S_{n}^{\alpha}+v S_{n}^{\beta}\right\}$.

Definition 4.1.2. The linear space of $S$ satisfying the genus one property is called the conformal block on the torus.

We compare properties (1)-(6) of correlation functions on the sphere with the properties of correlation functions on the sphere. We assume for simplicity that $V$ satisfies the property that $\operatorname{dim} V_{0}=1$ and $1 \notin L_{1} V$. Let $1^{\prime} \in V^{\prime}=\bigoplus_{n=0}^{\infty} V_{n}^{*}$ satisfy $\left\langle 1^{\prime}, 1\right\rangle=1$ and $\left\langle 1^{\prime}, a\right\rangle=0$ if $\operatorname{deg} a \geq 1$. Consider the power series

$$
\left\langle 1^{\prime}, Y\left(a_{1}, z_{1}\right) \ldots Y\left(a_{n}, z_{n}\right) 1\right\rangle
$$

by Proposition 1.4.1, it converges on the domain

$$
\left|z_{1}\right|>\cdots>\left|z_{n}\right|>0
$$

to a rational function

$$
\left(1^{\prime}, Y\left(a_{1}, z_{1}\right) \ldots Y\left(a_{n}, z_{n}\right) 1\right) .
$$

Since 1 satisfies the property $a_{i}(k) 1=0$ for $k \geq 0, z_{i}=0$ is not a pole; (4.1.6) has possible poles only at $z_{i}=z_{j}$. Functions (4.1.6) satisfy the following properties $(1)^{\prime}-(6)^{\prime}$ analogous to properties (1)-(6) in Definition 4.1.1.

$(1)^{\prime}\left(1^{\prime}, Y\left(a_{1}, z_{1}\right) \ldots Y\left(a_{n}, z_{n}\right) 1\right)$ is linear for each $a_{i}$.

$(2)^{\prime}$ For every permutation $\sigma$ of $n$ letters,

$$
\left(1^{\prime}, Y\left(a_{\sigma_{1}}, z_{\sigma_{1}}\right) \ldots Y\left(a_{\sigma_{n}}, z_{\sigma_{n}}\right) 1\right)=\left(1^{\prime}, Y\left(a_{1}, z_{1}\right) \ldots Y\left(a_{n}, z_{n}\right) 1\right) .
$$

$$
\left(1^{\prime}, Y(1, z) Y\left(a_{1}, z_{1}\right) \ldots Y\left(a_{n}, z_{n}\right) 1\right)=\left(1^{\prime}, Y\left(a_{1}, z_{1}\right) \ldots Y\left(a_{n}, z_{n}\right) 1\right) .
$$

$$
\left(1^{\prime}, Y\left(L_{-1} a_{1}, z_{1}\right) \ldots Y\left(a_{n}, z_{n}\right) 1\right)=\frac{d}{d z_{1}} S\left(1^{\prime}, Y\left(a_{1}, z_{1}\right) \ldots Y\left(a_{n}, z_{n}\right) 1\right) .
$$

$$
\begin{aligned}
& \int_{C}\left(1^{\prime}, Y(a, w) Y\left(a_{1}, z_{1}\right) \ldots Y\left(a_{n}, z_{n}\right) 1\right)\left(w-z_{1}\right)^{k} d w \\
& =\left(1^{\prime}, Y\left(a(k) a_{1}, z_{1}\right) \ldots Y\left(a_{n}, z_{n}\right) 1\right),
\end{aligned}
$$

where $C$ is a contour of $w$ surrounding $z_{1}$. 
$(6)^{\prime}$ If $a_{i}(i=1, \ldots, n)$ are highest weight vectors for the Virasoro algebra with degree $\operatorname{deg} a_{i}$, write

$$
S=\left(1^{\prime}, Y\left(\omega, w_{1}\right) \ldots Y\left(\omega, w_{m}\right) Y\left(a_{1}, z_{1}\right) \ldots Y\left(a_{n}, z_{n}\right) 1\right)
$$

then

$$
\begin{aligned}
& \left(1^{\prime}, Y(\omega, w) Y\left(\omega, w_{1}\right) \ldots Y\left(\omega, w_{m}\right) Y\left(a_{1}, z_{1}\right) \ldots Y\left(a_{n}, z_{n}\right) 1\right) \\
& =\sum_{k=1}^{n} \frac{1}{w-z_{k}} \frac{d}{d z_{k}} S+\sum_{k=1}^{m} \frac{1}{w-w_{k}} \frac{d}{d w_{k}} S \\
& +\sum_{k=1}^{n} \frac{\operatorname{deg} a_{k}}{\left(w-z_{k}\right)^{2}} S+\sum_{k=1}^{m} \frac{2}{\left(w-w_{k}\right)^{2}} S \\
& +\frac{c}{2} \sum_{k=1}^{m} \frac{1}{\left(w-w_{k}\right)^{4}}\left(1^{\prime}, Y\left(\omega, w_{1}\right) \ldots Y\left({\widehat{\left(\omega, w_{k}\right.}}^{2} \ldots Y\left(a_{1}, z_{1}\right) \ldots Y\left(a_{n}, z_{n}\right) 1\right),\right.
\end{aligned}
$$

\left. where $Y{\widehat{\left(\omega, w_{k}\right.}}\right)$ means the term is omitted.

Properties $(1)^{\prime},(3)^{\prime}$ and $(4)^{\prime}$ are obvious, $(2)^{\prime}$ and $(5)^{\prime}$ are proved in Proposition 1.4.1, and $(6)^{\prime}$ follows from (1.4.3).

Comparing (6) with $(6)^{\prime}$, we note that (6) has an additional term $2 \pi i \frac{d}{d \tau} S$. This difference reflects the fact that the moduli space for a Riemann surface of genus zero is a single point, while the moduli space for a genus one Rieman surface is one dimensional.

Having defined correlation functions on the torus, an immediate question is to prove the existence and to find the dimension of the conformal block. Our ultimate goal is to prove that for a rational vertex operator algebra satisfying finiteness condition $C$ (which is given in Section 4.4) the dimension of the conformal block on the torus is the same as the number of its irreducible representations. The main purpose of this section is to prove that for $V$ satisfying finiteness condition $C$ and a $V$-module $M=\bigoplus_{n=0}^{\infty} M_{n}$ with $\operatorname{dim} M_{n}<\infty$ for all $n$ and $\left.L_{0}\right|_{M_{n}}=(n+h) I d$ for some constant $h$, the power series

$$
\left.\operatorname{tr}\right|_{M} Y\left(a_{1}, z_{1}\right) \ldots Y\left(a_{n}, z_{n}\right) q^{L_{0}}
$$

converges on the domain

$$
|q|<\left|z_{n}\right|<\cdots<\left|z_{1}\right|<1
$$

for every $n$ and all insertions $Y\left(a_{1}, z_{1}\right), \ldots, Y\left(a_{n}, z_{n}\right)$, and the limits give rise to the maps $\left\{S_{n}\right\}$ satisfying the genus one property, or in other words, $M$ gives a vector in the conformal block on the torus. (In Section 5 we will prove that if $V$ is rational and satisfies a certain finiteness condition, the conformal block on the torus has a basis given by its irreducible modules.)

Let us now perform a formal computation to give a heuristic proof that a certain normalization of the limit of (4.1.7) is indeed a multivariable elliptic function.

Given a vertex operator algebra $V$ and a $V$-module $M=\bigoplus_{n \in \mathbf{N}} M_{n}$ such that $\operatorname{dim} M_{n}<\infty$ for every $n \in \mathbf{N}$ and $\left.L_{0}\right|_{M_{n}}=(n+h) I d$ ( $h$ is a constant), then 
the $q$-trace (4.1.7) is in $q^{h} \mathbf{C}\left[\left[z_{1}, z_{1}^{-1} ; \ldots ; z_{n}, z_{n}^{-1} ; q\right]\right]$. Assuming (4.1.7) converges "nicely" , since the coefficient of $q^{h+n}$ is a rational function of variables $z_{i}$ and is independent of the ordering of $Y\left(a_{i}, z_{i}\right)$ (Proposition 1.4.1), we have

$$
\begin{aligned}
& \left.\operatorname{tr}\right|_{M} Y\left(a_{1}, z_{1}\right) Y\left(a_{2}, z_{2}\right) \ldots Y\left(a_{n}, z_{n}\right) q^{L_{0}} \\
& =\left.\operatorname{tr}\right|_{M} Y\left(a_{2}, z_{2}\right) \ldots Y\left(a_{n}, z_{n}\right) Y\left(a_{1}, z_{1}\right) q^{L_{0}} .
\end{aligned}
$$

It is easy to prove that $Y\left(a_{1}, z_{1}\right) q^{L_{0}}=q^{-\operatorname{deg} a_{1}} q^{L_{0}} Y\left(a_{1}, z_{1} q^{-1}\right)$. Thus we have

$$
\begin{aligned}
& \left.\operatorname{tr}\right|_{M} Y\left(a_{2}, z_{2}\right) \ldots Y\left(a_{n}, z_{n}\right) Y\left(a_{1}, z_{1}\right) q^{L_{0}} \\
& =\left.q^{-\operatorname{deg} a_{1}} \operatorname{tr}\right|_{M} Y\left(a_{2}, z_{2}\right) \ldots Y\left(a_{n}, z_{n}\right) q^{L_{0}} Y\left(a_{1}, z_{1} q^{-1}\right) \\
& =\left.q^{-\operatorname{deg} a_{1}} \operatorname{tr}\right|_{M} Y\left(a_{1}, z_{1} q^{-1}\right) Y\left(a_{2}, z_{2}\right) \ldots Y\left(a_{n}, z_{n}\right) q^{L_{0}} .
\end{aligned}
$$

(4.1.8) and (4.1.9) yield

$$
\begin{aligned}
& \left.\operatorname{tr}\right|_{M} Y\left(a_{1}, z_{1}\right) Y\left(a_{2}, z_{2}\right) \ldots Y\left(a_{n}, z_{n}\right) q^{L_{0}} \\
& \quad=\left.q^{-\operatorname{deg} a_{1}} \operatorname{tr}\right|_{M} Y\left(a_{1}, z_{1} q^{-1}\right) Y\left(a_{2}, z_{2}\right) \ldots Y\left(a_{n}, z_{n}\right) q^{L_{0}} .
\end{aligned}
$$

We set

$$
F\left(\left(a_{1}, z_{1}\right), \ldots,\left(a_{n}, z_{n}\right), q\right)=\left.z_{1}^{\operatorname{deg} a_{1}} \ldots z_{n}^{\operatorname{deg} a_{n}} \operatorname{tr}\right|_{M} Y\left(a_{1}, z_{1}\right) \ldots Y\left(a_{n}, z_{n}\right) q^{L_{0}}
$$

then (4.1.10) is equivalent to

$$
F\left(\left(a_{1}, z_{1}\right),\left(a_{2}, z_{2}\right), \ldots,\left(a_{n}, z_{n}\right), q\right)=F\left(\left(a_{1}, z_{1} q^{-1}\right),\left(a_{2}, z_{2}\right), \ldots,\left(a_{n}, z_{n}\right), q\right) .
$$

We make a change of variable

$$
S\left(\left(a_{1}, z_{1}\right), \ldots,\left(a_{n}, z_{n}\right), \tau\right)=F\left(\left(a_{1}, e^{2 \pi i z_{1}}\right), \ldots,\left(a_{n}, e^{2 \pi i z_{n}}\right), e^{2 \pi i \tau}\right) e^{-\frac{2 \pi i c \tau}{24}},
$$

then (4.1.12) means that $S\left(\left(a_{1}, z_{1}\right),\left(a_{2}, z_{2}\right), \ldots,\left(a_{n}, z_{n}\right), \tau\right)$ is an elliptic function for $z_{1}$ with periods 1 and $\tau$. Since (4.1.12) is independent of the ordering of $Y\left(a_{i}, z_{i}\right)$, we conclude that $S$ is elliptic for each variable $z_{i}$.

To prove rigorously that (4.1.7) converges and $S\left(\left(a_{1}, z_{1}\right),\left(a_{2}, z_{2}\right), \ldots,\left(a_{n}, z_{n}\right), \tau\right)$ as in (4.1.13) satisfies the genus one property, we will proceed in three steps. In Step 1, we derive a recurrent formula which expresses the $(n+1)$-point $q$-trace in terms of the $n$-point trace (this step is accomplished in Section 4.3, Proposition 4.3.4). The recurrent formula can be regarded as a generalization of (1.4.3) in the genus one case; the method we use is also similar to (1.4.3). The recurrent formula reduces the convergence of the $n$-point $q$-trace to the convergence of the 1 -point $q$ trace. In the genus zero case (see the proof of Proposition 1.4.1), the convergence of a 1-point function is automatic, but in this genus one case, we need to do additional work to prove the convergence of the 1-point $q$-trace, which is done in Step 2. In Step 2 (Section 4.4) we prove that a certain finiteness condition on $V$ implies the existence of an ordinary differential equation satisfied by the 1-point $q$-trace. This differential equation implies that the 1-point $q$-trace converges by standard results on differential equations. In the process of Steps 1 and 2, we will automatically prove the properties (1)-(6), which will be written in detail in Step 3 (Theorem 4.4.3). 
4.2. Coordinate transformation. Let $\{V, Y(, z), 1, \omega\}$ be a vertex operator algebra, and let $W$ be a vector space isomorphic to $V$ by an isomorphism $T: V \rightarrow$ $W$. Then $T$ passes the vertex operator algebra structure of $V$ to $W$ in a trivial way: for $a \in W$,

$$
Y_{T}(a, z)=T Y\left(T^{-1} a, z\right) T^{-1} \in \operatorname{End}(W)\left[\left[z, z^{-1}\right]\right]
$$

$T 1$ and $T \omega$ are the identity element and the Virasoro element of $W$ respectively. In particular, this applies to the case when $T$ is a linear isomorphism to itself.

We discuss the case when $T$ is an isomorphism of $V$ associated to a coordinate transformation. A formal power series or a holomorphic map $\phi(z)=\sum_{n=1}^{\infty} k_{n} z^{n}$ near $0 \in \mathbf{C}$ is called a coordinate transformation if $k_{1} \neq 0$. To such a $\phi(z)$, we attach a linear isomorphism $T_{\phi}$ of $V$ as follows. We write

$$
\phi(z)=\exp \left(\sum_{i=0}^{\infty} l_{i} z^{i+1} \frac{d}{d z}\right) z .
$$

The $l_{i}$ 's are unique if we further require that $0 \leq i m\left(l_{0}\right) \leq 2 \pi$. Then $T_{\phi}$ is defined as

$$
T_{\phi}=\exp \left(\sum_{i=0}^{\infty} l_{i} L_{i}\right) .
$$

In the case that $T$ is the linear isomorphism associated to the local coordinate transformation $\phi(z)=\exp (2 \pi i z)-1$, we derive in this subsection a closed formula for $T Y\left(T^{-1} a, z\right) T^{-1}$. The reason we study this particular coordinate transformation is the following. Taking the $q$-trace of the products of vertex operators

$$
\left.\operatorname{tr}\right|_{M} Y\left(a_{1}, z_{1}\right) \ldots Y\left(a_{n}, z_{n}\right) q^{L_{0}}
$$

corresponds the geometric process of identifying the points $z$ and $q z$ in $\mathbf{C}-\{0\}$ to obtain the torus $\mathbf{C}-\{0\} /\left\{w \sim w q^{n}\right\}$ (here we consider $q$ as a non-zero complex number). The $z_{i}$ 's in $(4.2 .1)$ are the local coordinates on $\mathbf{C}-\{0\} /\left\{w \sim w q^{n}\right\}$ inherented from $\mathbf{C}-\{0\}$. However it is convenient to look at the torus in the picture $\mathbf{C} /\{m \tau+n\}(i m \tau>0)$ and use the local coordinate inherented from $\mathbf{C}$. For $q=e^{2 \pi i \tau}, \mathbf{C}-\{0\} /\left\{w \sim w q^{n}\right\}$ and $\mathbf{C} /\{m \tau+n\}$ are the same torus; two local coordinates are related by $w=\phi(z)=e^{2 \pi i z}-1$. The formula for $T_{\phi} Y\left(T_{\phi}^{-1} a, z\right) T_{\phi}^{-1}$ (we transport the local coordinates so that $z=0$ corresponds to $w=0$ ) will be derived as needed in computations in Section 4.3.

Theorem 4.2.1. Let $\{V, Y(, z), 1, \omega\}$ a vertex operator algebra, $k$ a nonzero complex number. We define a linear map from $V$ to $\operatorname{End}(V)\left[\left[z, z^{-1}\right]\right]$ by

$$
Y[a, z]_{k}=Y\left(a, e^{k z}-1\right) e^{k z \operatorname{deg} a}
$$

( $a \in V$ is homogeneous). Then this defines a new vertex operator algebra structure on $V$ with 1 as the identity element and $\widetilde{\omega}_{k}=k^{2}(\omega-c / 24)$ as the Virasoro element.

We first check that (4.2.2) is well-defined. We write $Y[a, z]_{k}=\sum_{n \in \mathbf{Z}} a[n]_{k} z^{-n-1}$, then it is easy to prove using an extension of (1.1.3) that

$$
a[m]_{k}=k^{-m-1} \operatorname{Res}_{z}\left(Y(a, z)(\ln (1+z))^{m}(1+z)^{\operatorname{deg} a-1}\right) .
$$


Write $(\ln (1+z))^{m}(1+z)^{\operatorname{deg} a-1}=\sum_{i \geq m} c_{i} z^{i}$; then $a[m]_{k}=\sum_{i \geq m} c_{i} a(i)$, so $a[m]_{k}$ is well-defined and satisfies that, for given $a, b \in V, a[m]_{k} b=0$ for $m$ sufficiently large.

Proof. For simplicity, we will write $a[n]$ for $a[n]_{k}$ and $Y[a, z]=Y[a, z]_{k}$ in the proof.

We first prove the Jacobi identity:

$$
\begin{aligned}
& \operatorname{Res}_{z-w}\left(Y[Y[a, z-w] b, w] \iota_{w, z-w}(z-w)^{m} z^{n}\right) \\
& =\operatorname{Res}_{z}\left(Y[a, z] Y[b, w] \iota_{z, w}(z-w)^{m} z^{n}\right)-\operatorname{Res}_{z}\left(Y[b, w] Y[a, z] \iota_{w, z}(z-w)^{m} z^{n}\right) .
\end{aligned}
$$

For simplicity, we only prove the case $n=0$. This is equivalent to proving

$$
(a[m] b)[n]=(-1)^{i}\left(\begin{array}{c}
m \\
i
\end{array}\right) a[m-i] b[n+i]-(-1)^{m+i}\left(\begin{array}{c}
m \\
i
\end{array}\right) b[m+n-i] a[i] .
$$

Let $(\ln (1+z))^{m}(1+z)^{\operatorname{deg} a-1}=\sum_{i \in \mathbf{N}} c_{m+i} z^{m+i}$, let $v \in V, v^{\prime} \in V^{\prime}$ ( $V^{\prime}$ is the restricted dual of $V)$, and consider $\left\langle v^{\prime},(a[m] b)[n] v\right\rangle$. We have

$$
\begin{aligned}
\left\langle v^{\prime},(a[m] b)[n] v\right\rangle \\
=\sum_{i \geq m} c_{i}\left(v^{\prime},(a(i) b)[n] v\right) \\
=k^{-n-1} \sum_{i \geq m} c_{i} \int_{C}\left(v^{\prime}, Y(a(i) b, w) v\right)(\ln (1+w))^{n}(1+w)^{\operatorname{deg} a+\operatorname{deg} b-i-2} d w \\
=k^{-n-1} \sum_{i \geq m} c_{i} \int_{C_{1}} \int_{C_{2}}\left(v^{\prime}, Y(Y(a, z-w) b, w) v\right) \\
\quad \cdot(z-w)^{i}(\ln (1+w))^{n}(1+w)^{\operatorname{deg} a+\operatorname{deg} b-i-2} d z d w \\
=k^{-n-m-2} \int_{C_{1}} \int_{C_{2}}\left(v^{\prime}, Y(a(z-w) b, w) v\right) \\
\quad \cdot\left(\ln \left(1+\frac{z-w}{1+w}\right)\right)^{m}\left(1+\frac{z-w}{1+w}\right)^{\operatorname{deg} a-1}(\ln (1+w))^{n}(1+w)^{\operatorname{deg} a+\operatorname{deg} b-2} d z d w \\
=k^{-n-m-2} \int_{C_{1}} \int_{C_{2}}\left(v^{\prime}, Y(Y(a, z-w) b, w) v\right) \\
\quad \cdot(\ln (1+z)-\ln (1+w))^{m}(\ln (1+w))^{n}(1+z)^{\operatorname{deg} a-1}(1+w)^{\operatorname{deg} b-1} d z d w,
\end{aligned}
$$

where $\left(v^{\prime}, Y(Y(a, z-w) b, w) v\right)=\left(v^{\prime}, Y(a, z) Y(b, w) v\right)$, etc., are rational functions with poles possible only at $z=w, z=0$ and $w=0$ (see Proposition 1.4 .1 and Proposition 1.4.2), $C$ is the contour of $w$ surrounding $0, C_{1}$ is the contour of $w$ containing the pole 0 , and $C_{2}$ is the contour of $z$ containing the pole $w$. Denote

$$
k^{-m-n-2}(\ln (1+z)-\ln (1+w))^{m}(\ln (1+w))^{n}(1+z)^{\operatorname{deg} a-1}(1+w)^{\operatorname{deg} b-1}
$$

by $F(z, w)$. By the Cauchy formula for contour integrals, we obtain

$$
\begin{aligned}
& \left(v^{\prime}(a[m] b)[n] v\right) \\
& =\int_{C_{1}^{\prime \prime}} \int_{C_{2}^{\prime \prime}}\left(v^{\prime}, Y(a, z) Y(b, w) v\right) F(z, w) d w d z \\
& \quad-\int_{C_{2}^{\prime}} \int_{C_{1}^{\prime}}\left(v^{\prime}, Y(b, w) Y(a, z) v\right) F(z, w) d z d w \\
& =(-1)^{i}\left(\begin{array}{c}
m \\
i
\end{array}\right) a[m-i] b[n+i]-(-1)^{m+i}\left(\begin{array}{c}
m \\
i
\end{array}\right) b[m+n-i] a[i],
\end{aligned}
$$


where the contour $C_{1}^{\prime \prime}$ of $z$ contains poles $w$ and 0 , the contour $C_{2}^{\prime \prime}$ of $w$ contains only 0 ; and the the contour $C_{2}^{\prime}$ of $w$ contains poles $z$ and 0 , the contour $C_{1}^{\prime}$ of $z$ contains only 0 . In the last step we used the fact that $|\ln (1+z)|>|\ln (1+w)|$ for $|z|>|w|$ and $|z|,|w|$ sufficiently small, so

$$
(\ln (1+z)-\ln (1+w))^{m}=\sum_{i=0}^{\infty}(-1)^{i}\left(\begin{array}{c}
m \\
i
\end{array}\right)(\ln (1+z))^{m-i}(\ln (1+w))^{i} .
$$

Thus the term $\int_{C_{1}} \int_{C_{2}} \ldots$ in (4.2.5) equals $(-1)^{i}\left(\begin{array}{c}m \\ i\end{array}\right) a[m-i] b[n+i]$. Similarly the term $\int_{C_{1}} \int_{C_{2}} \ldots$ in $(4.2 .5)$ equals $(-1)^{m+i}\left(\begin{array}{c}m \\ i\end{array}\right) b[m+n-i] a[i]$. This proves the Jacobi identity.

$Y[1, z]=Y\left(1, e^{z}-1\right)=1$, so 1 satisfies the axiom for the identity.

To prove that $\widetilde{\omega}_{k}=k^{2}(\omega-c / 24)$ is the Virasoro element, set

$$
Y\left[\widetilde{\omega}_{k}, z\right]_{k}=\sum_{n=-\infty}^{\infty} L[n]_{k} z^{-n-2}
$$

(we will write $L[n]$ for $L[n]_{k}$ in the rest of the proof). We need to prove that

$$
Y[L[-1] a, z]=\frac{d}{d z} Y[a, z]
$$

and

$$
[L[m], L[n]]=(m-n) L[m+n]+\frac{m^{3}-m}{12} \delta_{m+n, 0} c .
$$

Note that

$$
L[-1]=\widetilde{\omega}_{k}[0]=k^{2}\left(\omega-\frac{c}{24}\right)[0]_{k}=k^{2} \omega[0]=k\left(L_{-1}+L_{0}\right) .
$$

So we have

$$
\begin{aligned}
& (L[-1] a)[n] \\
& =k\left(L_{-1} a\right)[n]+k\left(L_{0} a\right)[n] \\
& =k^{-n} \operatorname{Res}_{z}\left(Y\left(L_{-1} a, z\right)(\ln (1+z))^{n}(1+z)^{\operatorname{deg} a}\right)+k \operatorname{deg} a a[n] \\
& =k^{-n} \operatorname{Res}_{z}\left(\frac{d}{d z} Y(a, z)(\ln (1+z))^{n}(1+z)^{\operatorname{deg} a}\right)+k \operatorname{deg} a a[n] \\
& =k^{-n} \operatorname{Res}_{z}\left(-Y(a, z)\left(n(\ln (1+z))^{n-1}(1+z)^{\operatorname{deg} a-1}\right.\right. \\
& \left.\left.\quad \quad-\operatorname{deg} a(\ln (1+z))^{n}(1+z)^{\operatorname{deg} a-1}\right)\right)+k \operatorname{deg} a a[n] \\
& =-n a[n-1] .
\end{aligned}
$$

(4.2.8) is equivalent to (4.2.4).

The Jacobi identity for vertex operators $Y[a, z]$ implies (1.2.11). In particular, we have

$$
\left[L[m], Y\left[\widetilde{\omega}_{k}, z\right]\right]=\sum_{i \geq-1}\left(\begin{array}{c}
m+1 \\
i+1
\end{array}\right) Y\left[L[i] \widetilde{\omega}_{k}, z\right] z^{m-i}
$$


then (4.2.7) follows from (4.2.6) (4.2.9) and the facts that

$$
L[0] \widetilde{\omega}=2 \widetilde{\omega}, \quad L[1] \widetilde{\omega}=0, \quad L[2] \widetilde{\omega}=\frac{1}{2} c, \quad L[k] \widetilde{\omega}=0 \quad \text { for } \quad k \geq 3 .
$$

Note that $L[0]=L_{0}+\sum_{i>1} k_{i} L_{i}$ for some constants $k_{i}$, so $V$ is a direct sum of eigenspaces of $L[0]$ with eigenvalues $n \in \mathbf{N}$. The other axioms of vertex operator algebras are trivially satisfied.

Next we prove that if $V$ satisfies a certain condition, then the vertex operator algebra structure in Theorem 4.2.1 is nothing but the structure given by the linear isomorphism associated to the coordinate transformation $\phi(z)=e^{k z}-1$.

Theorem 4.2.2. If $V$ is spanned by elements of the form $L_{-i_{1}} \ldots L_{-i_{n}}$ a for a satisfying $L_{i} a=0(i>0)$, then the linear map $T$ associated to the coordinate transformation $\phi(z)=e^{k z}-1$ gives an isomorphism of vertex operator algebras from $\{V, Y(, z), 1, \omega\}$ to $\left\{V, Y[, z]_{k}, 1, \widetilde{\omega}_{k}\right\}$.

Proof.. Let $\left\{V, Y_{T}(, z), T 1, T \omega\right\}$ (where $\left.Y_{T}(a, z)=T Y\left(T^{-1} a, z\right) T^{-1}\right)$ be the vertex operator algebra induced from $T$. It suffices to prove that

$$
Y_{T}(a, z)=Y[a, z]_{k},
$$

$T 1=1$ and $T \omega=\widetilde{\omega}_{k}$.

We first recall some basic facts about the commutation relation of the Virasoro algebra and the vertex operators for primary fields. Recall that the space $\mathcal{P}_{n}(V)$ of primary fields of degree $n$ is defined as

$$
\mathcal{P}_{n}(V)=\left\{a \in V \mid L_{i} a=0 \text { for } i>0, L_{0} a=n a\right\} .
$$

For $a \in \mathcal{P}_{n}(V)$, using (1.2.11) and (1.2.12), we have the commutation relation

$$
\left[L_{m}, Y(a, z)\right]=\left(n(m+1) z^{m}+z^{m+1} \frac{d}{d z}\right) Y(a, z) .
$$

The commutation relation of $L_{m}$ and $Y(\omega, z)$ is close to (4.2.11) with $n=2$ except for the central term:

$$
\left[L_{m}, Y(\omega, z)\right]=\left(2(m+1) z^{m}+z^{m+1} \frac{d}{d z}\right) Y(\omega, z)+\frac{\left(m^{3}-m\right) c}{12} z^{m-2} .
$$

Note that (4.2.11) is similar to the formula for the Lie derivative of a local $n$ differential $f(z)(d z)^{n}$ on a Riemann surface with respect to the holomorphic vector field $z^{m+1} \frac{d}{d z}$ :

$$
\nabla_{z^{m+1} \frac{d}{d z}} f(z)(d z)^{n}=\left((m+1) n z^{m}+z^{m+1} \frac{d}{d z}\right) f(z)(d z)^{n} .
$$

So $Y(a, z)$ has a covariance property similar to that of an $n$-differential. So for a local coordinate transformation $\phi(z)$ and its associated linear isomorphism $T_{\phi}$ defined as above, we have the relations

$$
\begin{aligned}
& T_{\phi} Y(a, z) T_{\phi}^{-1}=Y(a, \phi(z))\left(\phi^{\prime}(z)\right)^{n} \quad \text { for } a \in \mathcal{P}_{n}(V), \\
& T_{\phi} Y(\omega, z) T_{\phi}^{-1}=Y(\omega, \phi(z))\left(\phi^{\prime}(z)\right)^{2}+\frac{1}{12}\{\phi(z), z\} c .
\end{aligned}
$$


Here $\{\phi(z), z\}=\frac{\phi^{\prime \prime \prime}(z)}{\phi^{\prime}(z)}-\frac{3}{2}\left(\frac{\phi^{\prime \prime}(z)}{\phi^{\prime}(z)}\right)^{2}$ is the Schwarzian derivative of $\phi(z)$. The additional term $\frac{1}{12}\{\phi(z), z\} c$ in (4.2.14) is caused by the central term in (4.2.12).

Now we take $\phi(z)=e^{k z}-1$. A direct computation shows that $\{\phi(z), z\}=-\frac{k^{2}}{2}$. This is easy to see that $T 1=1$. To prove $T \omega=\widetilde{\omega}_{k}=k^{2}\left(\omega-\frac{1}{24} c\right)$, we apply $(4.2 .14)$ to 1 and take the constant term to get the needed identity. (4.2.13) implies that (4.2.10) is true for $a$ primary, and (4.2.14) implies (4.2.10) is true for $a=\widetilde{\omega}_{k}$.

Note that since two copies of Virasoro algebras $\left\{L_{n}\right\}$ and $\{L[n]\}$ satisfy

$$
L[n]=k^{-n} L_{n}+\sum_{i \geq 1} l_{n, i} L_{n+i} \quad \text { for some } l_{n, i}
$$

and so

$$
L_{n}=k^{n} L[n]+\sum_{i \geq 1} l_{n, i}^{\prime} L[n+i] \quad \text { for some } l_{n, i}
$$

it follows that the highest weight vectors (or primary fields) in $V$ for both Virasoro algebras are the same.

We have proved that two vertex operator algebra structures on $V$,

$$
\left\{V, Y_{T}(, z), T 1, T \omega\right\} \quad \text { and } \quad\left\{V, Y[, z]_{k}, 1, \widetilde{\omega}_{k}\right\}
$$

have the same identity element 1 and the Viraroso element $\widetilde{\omega}_{k}$, and $Y[a, z]_{k}=$ $Y_{T}(a, z)$ is true for $a$ primary and $a=\widetilde{\omega}_{k}$ (by (4.2.13) and (4.2.14)). This together with the Jacobi identity further implies that $Y[a, z]_{k}=Y_{T}(a, z)$ is true if $a$ is of the form $L\left[-i_{1}\right] \ldots L\left[-i_{n}\right] b$ (b primary). Using the condition that $V$ is spanned by elements of the form $L_{-i_{1}} \cdots L_{-i_{n}} a$ for $a$ primary (which implies that $V$ is spanned by elements of the form $L\left[-i_{1}\right] \cdots L\left[-i_{n}\right] a$ for $a$ primary by $(4.2 .15)$ and (4.2.16)), we see that $Y[a, z]_{k}=Y_{T}(a, z)$ is true for every $a$ in $V$. This completes the proof that $\left\{V, Y_{T}(, z), T 1, T \omega\right\}$ and $\left\{V, Y[, z]_{k}, 1, \widetilde{\omega}_{k}\right\}$ are identical vertex operator structures on $V$.

Remark. It is not clear if the two vertex operator algebras in Theorem 4.2.2 are the same in general. For a general coordinate transformation $w=\phi(z), Y_{T_{\phi}}(a, z)$ does not have the simple formula that it does in the case $\phi(z)=e^{k z}-1$. A different proof of Theorem 4.2.1 is given in [Le].

In the rest of Section 4 , we will meet the case $k=2 \pi i$. We denote

$$
Y[a, z]=\sum_{n \in \mathbf{Z}} a[n] z^{-n-1}=Y\left(a, e^{2 \pi i z}-1\right) e^{2 \pi i z \operatorname{deg} a}
$$

SO

$$
a[m]=(2 \pi i)^{-m-1} \operatorname{Res}_{z}\left(Y(a, z)(\ln (1+z))^{m}(1+z)^{\operatorname{deg} a-1}\right),
$$

and we denote $\widetilde{\omega}=(2 \pi i)^{2}\left(\omega-\frac{c}{24}\right), \widetilde{\omega}[n+1]=L[n]$. Note that the gradation of the vertex operator algebra $\{V, Y[, z], 1, \widetilde{\omega}\}$ given by $L[0]$ is different from the old one; we denote this new gradation by $[\operatorname{deg}]$. So $[\operatorname{deg} a]=n$ means $L[0] a=[\operatorname{deg}](a)$. 
4.3. Identities for the $q$-trace of vertex operators. Given a vertex operator algebra $V$ and a $V$-module $M=\bigoplus_{n \in \mathbf{N}} M_{n}$ with $\operatorname{dim}\left(M_{n}\right)<\infty$ and $\left.L_{0}\right|_{M_{n}}=n+h$ for some constant $h$, the $n$-point $q$-trace

$$
\left.\operatorname{tr}\right|_{M} Y\left(a_{1}, z_{1}\right) Y\left(a_{2}, z_{2}\right) \ldots Y\left(a_{n}, z_{n}\right) q^{L_{0}}
$$

is in $q^{h} \mathbf{C}\left[\left[z_{1}, z_{1}^{-1} ; \ldots ; z_{n}, z_{n}^{-1} ; q\right]\right]$. The object of this subsection is to derive a recurrent formula which expresses the $n$-point $q$-trace (4.3.1) in terms of the $(n-1)$-point trace, and to prove a vanishing condition for the 1-point $q$-trace. For convenience, we introduce the notation $F_{M}$ :

$$
\left.F_{M}\left(\left(a_{1}, z_{1}\right), \ldots,\left(a_{n}, z_{n}\right), q\right)\right)=\left.z_{1}^{\operatorname{deg} a_{1}} \ldots z_{n}^{\operatorname{deg} a_{n}} \operatorname{tr}\right|_{M} Y\left(a_{1}, z_{1}\right) \ldots Y\left(a_{n}, z_{n}\right) q^{L_{0}}
$$

for homogeneous $a_{i}(i=1, \ldots, n)$ and extend $F_{M}$ by linearity. For simplicity we will often omit the lower index $M$ in $F_{M}$ when no confusion should arise.

The following combinatorial lemma will be needed later; it may be proved by using generating functions.

Lemma 4.3.1. Let $c(m, i, s)$ be given by $(\underset{i}{\operatorname{deg} a-1+x})=\sum_{s=0}^{i} c(m, i, s) x^{s}$. Then

$$
\sum_{i=s}^{\infty} c(\operatorname{deg} a, i, s) z^{i}=\frac{1}{s !}[\ln (1+z)]^{s}(1+z)^{\operatorname{deg} a-1} .
$$

This lemma implies that

$$
\sum_{i=s}^{\infty} c(\operatorname{deg} a, i, s) a(i)=\frac{(2 \pi i)^{s+1}}{s !} a[s] .
$$

The following proposition reflects the cyclic property of trace.

Proposition 4.3.1. For every $a, a_{1}, \ldots, a_{n} \in V$

$$
\sum_{k=1}^{n} F\left(\left(a_{1}, z_{1}\right), \ldots,\left(a[0] a_{k}, z_{k}\right), \ldots,\left(a_{n}, z_{n}\right), q\right)=0 .
$$

Proof. Appling the cyclic property of the trace to each coefficient of $z_{1}^{i_{1}} \cdots z_{n}^{i_{n}} q^{n+h}$, we have

$$
\begin{aligned}
0= & \left.\operatorname{tr}\right|_{M}\left[a(\operatorname{deg} a-1), Y\left(a_{1}, z_{1}\right) \ldots Y\left(a_{n}, z_{n}\right)\right] q^{L_{0}} \\
= & \left.\operatorname{tr}\right|_{M} \sum_{k=1}^{n} \sum_{i=0}^{\infty}\left(\begin{array}{c}
\operatorname{deg} a-1 \\
i
\end{array}\right) z_{k}^{\operatorname{deg} a-1-i} Y\left(a_{1}, z_{1}\right) \ldots Y\left(a(i) a_{k}, z_{k}\right) \ldots Y\left(a_{n}, z_{n}\right) q^{L_{0}} \\
= & z_{1}^{-\operatorname{deg} a_{1}} \ldots z_{n}^{-\operatorname{deg} a_{n}} \sum_{k=1}^{n} \sum_{i=0}^{\infty}\left(\begin{array}{c}
\operatorname{deg} a-1 \\
i
\end{array}\right) . \\
& \cdot F\left(\left(a_{1}, z_{1}\right), \ldots,\left(a(i) a_{k}, z_{k}\right), \ldots,\left(a_{n}, z_{n}\right), q\right) \\
= & (2 \pi i) z_{1}^{-\operatorname{deg} a_{1}} \ldots z_{n}^{-\operatorname{deg} a_{n}} \sum_{k=1}^{n} F_{M}\left(\left(a_{1}, z_{1}\right), \ldots,\left(a[0] a_{k}, z_{k}\right), \ldots,\left(a_{n}, z_{n}\right), q\right) .
\end{aligned}
$$


Proposition 4.3.2. Let $P_{m}(z, q)$ be the formal power series defined in Section 3. We have

$$
\begin{aligned}
& F\left(\left(a_{1}, z_{1}\right), \ldots,\left(a_{j}, z_{j}\right),(a, w),\left(a_{j+1}, z_{j+1}\right), \ldots,\left(a_{n}, z_{n}\right), q\right) \\
& =z_{1}^{-\operatorname{deg} a_{1}} \ldots z_{n}^{-\operatorname{deg} a_{n}} \operatorname{tra}(\operatorname{deg} a-1) Y\left(a_{1}, z_{1}\right) Y\left(a_{2}, z_{2}\right) \ldots Y\left(a_{n}, z_{n}\right) q^{L_{0}} \\
& +\sum_{k=1}^{j} \sum_{m \in \mathbf{N}}\left(P_{m+1}\left(\frac{z_{k} q}{w}, q\right)-2 \pi i \delta_{m, 0}\right) F\left(\left(a_{1}, z_{1}\right), \ldots,\left(a[m] a_{k}, z_{k}\right), \ldots,\left(a_{n}, z_{n}\right), q\right) \\
& +\sum_{k=j+1}^{n} \sum_{m \in \mathbf{N}} P_{m+1}\left(\frac{z_{k}}{w}, q\right) F\left(\left(a_{1}, z_{1}\right), \ldots,\left(a[m] a_{k}, z_{k}\right), \ldots,\left(a_{n}, z_{n}\right), q\right) .
\end{aligned}
$$

Proof. For simplicity we only write the proof for the case $j=1$; the general case should be clear. We have

$$
\begin{aligned}
& F\left(\left(a_{1}, z_{1}\right),(a, w),\left(a_{2}, z_{2}\right), \ldots,\left(a_{n}, z_{n}\right), q\right) \\
& =w^{\operatorname{deg} a} z_{1}^{\operatorname{deg} a_{1}} \ldots z_{n}^{\operatorname{deg} a_{n}} \operatorname{tr} Y\left(a_{1}, z_{1}\right) Y(a, w) Y\left(a_{2}, z_{2}\right) \ldots Y\left(a_{n}, z_{n}\right) q^{L_{0}} \\
& =z_{1}^{\operatorname{deg} a_{1}} \ldots z_{n}^{\operatorname{deg} a_{n}} \sum_{k \in \mathbf{Z}} w^{-k} \operatorname{tr} Y\left(a_{1}, z_{1}\right) a(\operatorname{deg} a-1+k) \ldots Y\left(a_{n}, z_{n}\right) q^{L_{0}} .
\end{aligned}
$$

For $k \neq 0$, we have

$$
\begin{aligned}
& \operatorname{tr} Y\left(a_{1}, z_{1}\right) a(\operatorname{deg} a-1+k) \ldots Y\left(a_{n}, z_{n}\right) q^{L_{0}} \\
= & \operatorname{tr} Y\left(a_{1}, z_{1}\right)\left[a(\operatorname{deg} a-1+k), Y\left(a_{2}, z_{2}\right) \ldots Y\left(a_{n}, z_{n}\right)\right] q^{L_{0}} \\
& +\operatorname{tr} Y\left(a_{1}, z_{1}\right) \ldots Y\left(a_{n}, z_{n}\right) a(\operatorname{deg} a-1+k) q^{L_{0}} \\
= & \sum_{j=2}^{n} \sum_{i \in \mathbf{N}}\left(\begin{array}{c}
\operatorname{deg} a-1+k \\
i
\end{array}\right) z_{j}^{\operatorname{deg} a-1+k-i} \operatorname{tr} Y\left(a_{1}, z_{1}\right) \ldots Y\left(a(i) a_{j}, z_{j}\right) \ldots Y\left(a_{n}, z_{n}\right) q^{L_{0}} \\
& +\operatorname{tr} Y\left(a_{1}, z_{1}\right) \ldots Y\left(a_{n}, z_{n}\right) q^{L_{0}} a(\operatorname{deg} a-1+k) q^{k} \\
= & \sum_{j=2}^{n} \sum_{i \in \mathbf{N}}\left(\begin{array}{c}
\operatorname{deg} a-1+k \\
i
\end{array}\right) z_{j}^{\operatorname{deg} a-1+k-i} \operatorname{tr} Y\left(a_{1}, z_{1}\right) \ldots Y\left(a(i) a_{j}, z_{j}\right) \ldots Y\left(a_{n}, z_{n}\right) q^{L_{0}} \\
& +q^{k} \operatorname{tra}(\operatorname{deg} a-1+k) Y\left(a_{1}, z_{1}\right) \ldots Y\left(a_{n}, z_{n}\right) q^{L_{0}} \\
= & \sum_{j=2}^{n} \sum_{i \in \mathbf{N}}\left(\begin{array}{c}
\operatorname{deg} a-1+k \\
i
\end{array}\right) z_{j}^{\operatorname{deg} a-1+k-i} \operatorname{tr} Y\left(a_{1}, z_{1}\right) \ldots Y\left(a(i) a_{j}, z_{j}\right) \ldots Y\left(a_{n}, z_{n}\right) q^{L_{0}} \\
& +q^{k} \operatorname{tr} Y\left(a_{1}, z_{1}\right) a(\operatorname{deg} a-1+k) \ldots Y\left(a_{n}, z_{n}\right) q^{L_{0}} \\
& +q^{k} \sum_{i \in \mathbf{N}}\left(\begin{array}{c}
\operatorname{deg} a-1+k \\
i
\end{array}\right) z_{1}^{\operatorname{deg} a-1+k-i} \operatorname{tr} Y\left(a(i) a_{1}, z_{1}\right) \ldots Y\left(a_{n}, z_{n}\right) q^{L_{0}} .
\end{aligned}
$$


Solving for $\operatorname{tr} Y\left(a_{1}, z_{1}\right) a(\operatorname{deg} a-1+k) \ldots Y\left(a_{n}, z_{n}\right) q^{L_{0}}$ in the above identity, we obtain

$$
\begin{aligned}
& \operatorname{tr} Y\left(a_{1}, z_{1}\right) a(\operatorname{deg} a-1+k) \ldots Y\left(a_{n}, z_{n}\right) q^{L_{0}} \\
& =\frac{1}{1-q^{k}} \sum_{j=2}^{n} \sum_{i \in \mathbf{N}}\left(\begin{array}{c}
\operatorname{deg} a-1+k \\
i
\end{array}\right) z_{j}^{\operatorname{deg} a-1+k-i} \\
& \quad \cdot \operatorname{tr} Y\left(a_{1}, z_{1}\right) \ldots Y\left(a(i) a_{j}, z_{j}\right) \ldots Y\left(a_{n}, z_{n}\right) q^{L_{0}} \\
& \quad+\frac{q^{k}}{1-q^{k}} \sum_{i \in \mathbf{N}}\left(\begin{array}{c}
\operatorname{deg} a-1+k \\
i
\end{array}\right) z_{1}^{\operatorname{deg} a-1+k-i} \operatorname{tr} Y\left(a[i] a_{1}, z_{1}\right) \ldots Y\left(a_{n}, z_{n}\right) q^{L_{0}} .
\end{aligned}
$$

(where $\frac{1}{1-q^{k}}=\sum_{i=0}^{\infty} q^{k i}$ ). Using (4.3.5), we have

$F\left(\left(a_{1}, z_{1}\right),(a, w),\left(a_{2}, z_{2}\right), \ldots,\left(a_{n}, z_{n}\right), q\right)$

$$
\begin{aligned}
& =z_{1}^{\operatorname{deg} a_{1}} \ldots z_{n}^{\operatorname{deg} a_{n}} \operatorname{tr} Y\left(a_{1}, z_{1}\right) a(\operatorname{deg} a-1) Y\left(a_{2}, z_{2}\right) \ldots Y\left(a_{n}, z_{n}\right) q^{L_{0}} \\
& +z_{1}^{\operatorname{deg} a_{1}} \ldots z_{n}^{\operatorname{deg} a_{n}} \sum_{k \neq 0} w^{-k} \operatorname{tr} Y\left(a_{1}, z_{1}\right) a(\operatorname{deg} a-1+k) \ldots Y\left(a_{n}, z_{n}\right) q^{L_{0}} \\
& =z_{1}^{\operatorname{deg} a_{1}} \ldots z_{n}^{\operatorname{deg} a_{n}} \operatorname{tr} Y\left(a_{1}, z_{1}\right) a(\operatorname{deg} a-1) Y\left(a_{2}, z_{2}\right) \ldots Y\left(a_{n}, z_{n}\right) q^{L_{0}}
\end{aligned}
$$$$
+\sum_{k \neq 0} w^{-k} z_{1}^{\operatorname{deg} a_{1}} \ldots z_{n}^{\operatorname{deg} a_{n}} \frac{1}{1-q^{k}} \sum_{j=2}^{n} \sum_{i \in \mathbf{N}}\left(\begin{array}{c}
\operatorname{deg} a-1+k \\
i
\end{array}\right) z_{j}^{\operatorname{deg} a-1+k-i}
$$$$
\cdot \operatorname{tr} Y\left(a_{1}, z_{1}\right) \ldots Y\left(a(i) a_{j}, z_{j}\right) \ldots Y\left(a_{n}, z_{n}\right) q^{L_{0}}
$$$$
+\sum_{k \neq 0} w^{-k} z_{1}^{\operatorname{deg} a_{1}} \ldots z_{n}^{\operatorname{deg} a_{n}} \frac{q^{k}}{1-q^{k}} \sum_{i \in \mathbf{N}}\left(\begin{array}{c}
\operatorname{deg} a-1+k \\
i
\end{array}\right) z_{1}^{\operatorname{deg} a-1+k-i}
$$$$
\cdot \operatorname{tr} Y\left(a(i) a_{1}, z_{1}\right) \ldots Y\left(a_{n}, z_{n}\right) q^{L_{0}}
$$$$
=z_{1}^{\operatorname{deg} a_{1}} \ldots z_{n}^{\operatorname{deg} a_{n}} \operatorname{tr} Y\left(a_{1}, z_{1}\right) a(\operatorname{deg} a-1) Y\left(a_{2}, z_{2}\right) \ldots Y\left(a_{n}, z_{n}\right) q^{L_{0}}
$$$$
+\sum_{j=2}^{n} \sum_{i \in \mathbf{N}} \sum_{k=1}^{\infty}\left(\left(\begin{array}{c}
\operatorname{deg} a-1+k \\
i
\end{array}\right) \frac{1}{1-q^{k}}\left(\frac{z_{j}}{w}\right)^{k}+\left(\begin{array}{c}
\operatorname{deg} a-1-k \\
i
\end{array}\right) \frac{1}{1-q^{-k}}\left(\frac{z_{j}}{w}\right)^{-k}\right)
$$$$
\cdot F\left(\left(a_{1}, z_{1}\right), \ldots,\left(a(i) a_{j}, z_{j}\right), \ldots, q\right)
$$$$
+\sum_{i \in \mathbf{N}} \sum_{k=1}^{\infty}\left(\left(\begin{array}{c}
\operatorname{deg} a-1+k \\
i
\end{array}\right) \frac{1}{1-q^{k}}\left(\frac{q z_{1}}{w}\right)^{k}+\left(\begin{array}{c}
\operatorname{deg} a-1-k \\
i
\end{array}\right) \frac{1}{1-q^{-k}}\left(\frac{q z_{1}}{w}\right)^{-k}\right)
$$$$
\text { - } F\left(\left(a(i) a_{1}, z_{1}\right), \ldots, q\right)
$$$$
=z_{1}^{\operatorname{deg} a_{1}} \ldots z_{n}^{\operatorname{deg} a_{n}} \operatorname{tra}(\operatorname{deg} a-1) Y\left(a_{1}, z_{1}\right) Y\left(a_{2}, z_{2}\right) \ldots Y\left(a_{n}, z_{n}\right) q^{L_{0}}
$$$$
-2 \pi i F\left(\left(a[0] a_{1}, z_{1}\right),\left(a_{2}, z_{2}\right), \ldots,\left(a_{n}, z_{n}\right), q\right)
$$$$
+\sum_{j=2}^{n} \sum_{i \in \mathbf{N}} \sum_{k=1}^{\infty}\left(\left(\begin{array}{c}
\operatorname{deg} a-1+k \\
i
\end{array}\right) \frac{1}{1-q^{k}}\left(\frac{z_{j}}{w}\right)^{k}+\left(\begin{array}{c}
\operatorname{deg} a-1-k \\
i
\end{array}\right) \frac{1}{1-q^{-k}}\left(\frac{z_{j}}{w}\right)^{-k}\right)
$$ 


$$
\begin{aligned}
& \cdot F\left(\left(a_{1}, z_{1}\right), \ldots,\left(a(i) a_{j}, z_{j}\right), \ldots, q\right) \\
& +\sum_{i \in \mathbf{N}} \sum_{k=1}^{\infty}\left(\left(\begin{array}{c}
\operatorname{deg} a-1+k \\
i
\end{array}\right) \frac{1}{1-q^{k}}\left(\frac{q z_{1}}{w}\right)^{k}+\left(\begin{array}{c}
\operatorname{deg} a-1-k \\
i
\end{array}\right) \frac{1}{1-q^{-k}}\left(\frac{q z_{1}}{w}\right)^{-k}\right) \\
& \cdot F\left(\left(a(i) a_{1}, z_{1}\right), \ldots, q\right) .
\end{aligned}
$$

So our proposition is reduced to the identity

$$
\begin{aligned}
& \sum_{i \in \mathbf{N}} \sum_{k=1}^{\infty}\left(\left(\begin{array}{c}
\operatorname{deg} a-1+k \\
i
\end{array}\right) \frac{1}{1-q^{k}} x^{k}+\left(\begin{array}{c}
\operatorname{deg} a-1-k \\
i
\end{array}\right) \frac{1}{1-q^{-k}} x^{-k}\right) a(i) b \\
& =\sum_{m \in \mathbf{N}} P_{m+1}(x, z) a[m] b
\end{aligned}
$$

which is a corollary of Lemma 4.3.1.

However (4.3.4) doesn't accomplish our goal to express the $n+1$-point trace in terms of $n$-point traces, because of the appearance of the term

$$
z_{1}^{\operatorname{deg} a_{1}} \ldots z_{n}^{\operatorname{deg} a_{n}} \operatorname{tra}(\operatorname{deg} a-1) Y\left(a_{1}, z_{1}\right) Y\left(a_{2}, z_{2}\right) \ldots Y\left(a_{n}, z_{n}\right) q^{L_{0}}
$$

in (4.3.4). The elimination of this term is provided by the following proposition:

Proposition 4.3.3. We have

$$
\begin{aligned}
& F\left(\left(a[-1] a_{1}, z_{1}\right),\left(a_{2}, z_{2}\right), \ldots,\left(a_{n}, z_{n}\right), q\right) \\
& =z_{1}^{\operatorname{deg} a_{1}} \ldots z_{n}^{\operatorname{deg} a_{n}} \operatorname{tra}(\operatorname{deg} a-1) Y\left(a_{1}, z_{1}\right) Y\left(a_{2}, z_{2}\right) \ldots Y\left(a_{n}, z_{n}\right) q^{L_{0}} \\
& -\pi i F\left(\left(a[0] a_{1}, z_{1}\right),\left(a_{2}, z_{2}\right), \ldots,\left(a_{n}, z_{n}\right), q\right) \\
& +\sum_{k=1}^{\infty} \widetilde{G}_{2 k}(q) F\left(\left(a[2 k-1] a_{1}, z_{1}\right),\left(a_{2}, z_{2}\right), \ldots,\left(a_{n}, z_{n}\right), q\right) \\
& +\sum_{k=2}^{n} \sum_{m \in \mathbf{N}} P_{m+1}\left(\frac{z_{k}}{z_{1}}, q\right) F\left(\left(a_{1}, z_{1}\right), \ldots,\left(a[m] a_{k}, z_{k}\right), \ldots,\left(a_{n}, z_{n}\right), q\right) .
\end{aligned}
$$

Proof. Write $(1+z)^{\operatorname{deg} a-1}(\ln (1+z))^{-1}=\sum_{i \geq-1} c_{i} z^{i}$ (note that $\left.c_{-1}=1\right)$; then

$$
a[-1] a_{1}=\sum_{i \geq-1} c_{i} a(i) a_{1}
$$


and we have

$$
\begin{aligned}
F & \left.\left(a[-1] a_{1}, z_{1}\right),\left(a_{2}, z_{2}\right), \ldots,\left(a_{n}, z_{n}\right), q\right) \\
= & \sum_{i \geq-1} c_{i} z_{1}^{\operatorname{deg} a-1-i} z_{1}^{\operatorname{deg} a_{1}} \ldots z_{n}^{\operatorname{deg} a_{n}} \operatorname{tr} Y\left(a(i) a_{1}, z_{1}\right) Y\left(a_{2}, z_{2}\right) \ldots Y\left(a_{n}, z_{n}\right) q^{L_{0}} \\
= & \sum_{i \geq-1} c_{i} z_{1}^{\operatorname{deg} a-1-i} z_{1}^{\operatorname{deg} a_{1}} \ldots z_{n}^{\operatorname{deg} a_{n}} \operatorname{Res}_{w-z_{1}}\left(w-z_{1}\right)^{i} \\
& \left.\cdot \operatorname{tr}\right|_{M} Y\left(Y\left(a, w-z_{1}\right) a_{1}, z_{1}\right) Y\left(a_{2}, z_{2}\right) \ldots Y\left(a_{n}, z_{n}\right) q^{L_{0}} \\
= & \sum_{i \geq-1} c_{i} z_{1}^{\operatorname{deg} a-1-i} z_{1}^{\operatorname{deg} a_{1}} \ldots z_{n}^{\operatorname{deg} a_{n}} \operatorname{Res}_{w} \iota_{w, z_{1}}\left(w-z_{1}\right)^{i} \\
& \cdot \operatorname{tr} Y(a, w) Y\left(a_{1}, z_{1}\right) Y\left(a_{2}, z_{2}\right) \ldots Y\left(a_{n}, z_{n}\right) q^{L_{0}} \\
& -\sum_{i \geq-1} c_{i} z_{1}^{\operatorname{deg} a-1-i} z_{1}^{\operatorname{deg} a_{1}} \ldots z_{n}^{\operatorname{deg} a_{n}} \operatorname{Res}_{w} \iota_{z_{1}, w}\left(w-z_{1}\right)^{i} \\
& \cdot \operatorname{tr} Y\left(a_{1}, z_{1}\right) Y(a, w) Y\left(a_{2}, z_{2}\right) \ldots Y\left(a_{n}, z_{n}\right) q^{L_{0}} \\
= & \sum_{i \geq-1} c_{i} \operatorname{Res}_{w}\left(\iota_{w, z_{1}}\left(w-z_{1}\right)^{i} z_{1}^{\operatorname{deg} a-1-i} w^{-\operatorname{deg} a} F\left((a, w)\left(a_{1}, z_{1}\right), \ldots,\left(a_{n}, z_{n}\right), q\right)\right) \\
- & \sum_{i \geq-1} c_{i} \operatorname{Res}_{w}\left(\iota_{z_{1}, w}\left(w-z_{1}\right)^{i} z_{1}^{\operatorname{deg} a-1-i} w^{-\operatorname{deg} a} F\left(\left(a_{1}, z_{1}\right),(a, w), \ldots,\left(a_{n}, z_{n}\right), q\right)\right) .
\end{aligned}
$$

Now applying Proposition 4.3.2 to the expressions

$$
F\left((a, w),\left(a_{1}, z_{1}\right),\left(a_{2}, z_{2}\right), \ldots,\left(a_{n}, z_{n}\right), q\right)
$$

(the case $j=0$ ) and

$$
F\left(\left(a_{1}, z_{1}\right),(a, w),\left(a_{2}, z_{2}\right), \ldots,\left(a_{n}, z_{n}\right), q\right)
$$

(the case $j=1$ ), we see that in order to prove $(4.3 .7)$, we only need to show the following identities:

$$
\begin{aligned}
& \sum_{i \geq-1} c_{i} \operatorname{Res}_{w}\left(\iota_{w, z_{1}}\left(w-z_{1}\right)^{i} z_{1}^{\operatorname{deg} a-1-i} w^{-\operatorname{deg} a}\right) \\
& -\sum_{i \geq-1} c_{i} \operatorname{Res}_{w}\left(\iota_{z_{1}, w}\left(w-z_{1}\right)^{i} z_{1}^{\operatorname{deg} a-1-i} w^{-\operatorname{deg} a}\right) \\
& =1
\end{aligned}
$$

$$
\begin{aligned}
& \sum_{i \geq-1} c_{i} \operatorname{Res}_{w}\left(\iota_{w, z_{1}}\left(w-z_{1}\right)^{i} z_{1}^{\operatorname{deg} a-1-i} w^{-\operatorname{deg} a} P_{1}\left(\frac{z_{1}}{w}, q\right)\right) \\
& -\sum_{i \geq-1} c_{i} \operatorname{Res}_{w}\left(\iota_{z_{1}, w}\left(w-z_{1}\right)^{i} z_{1}^{\operatorname{deg} a-1-i} w^{-\operatorname{deg} a}\left(P_{1}\left(\frac{z_{1} q}{w}, q\right)-2 \pi i\right)\right) \\
& =-\pi i
\end{aligned}
$$


$(4.3 .10)$

$$
\begin{aligned}
& \sum_{i \geq-1} c_{i} \operatorname{Res}_{w}\left(\iota_{w, z_{1}}\left(w-z_{1}\right)^{i} z_{1}^{\operatorname{deg} a-1-i} w^{-\operatorname{deg} a} P_{m}\left(\frac{z_{1}}{w}, q\right)\right) \\
& -\sum_{i \geq-1} c_{i} \operatorname{Res}_{w}\left(\iota_{z_{1}, w}\left(w-z_{1}\right)^{i} z_{1}^{\operatorname{deg} a-1-i} w^{-\operatorname{deg} a}\left(P_{m}\left(\frac{z_{1} q}{w}, q\right)-2 \pi i \delta_{m, 0}\right)\right) \\
& =\widetilde{G}_{m}(q),
\end{aligned}
$$

where $m>1$ and $\widetilde{G}_{m}(q)=0$ for $m$ an odd number. and

$$
\begin{aligned}
& \sum_{i \geq-1} c_{i} \operatorname{Res}_{w}\left(\iota_{w, z_{1}}\left(w-z_{1}\right)^{i} z_{1}^{\operatorname{deg} a-1-i} w^{-\operatorname{deg} a} P_{m}\left(\frac{z_{k}}{w}, q\right)\right) \\
& -\sum_{i \geq-1} c_{i} \operatorname{Res}_{w}\left(\iota_{z_{1}, w}\left(w-z_{1}\right)^{i} z_{1}^{\operatorname{deg} a-1-i} w^{-\operatorname{deg} a} P\left(\frac{z_{k}}{w}, q\right)\right) \\
& =P_{m}\left(\frac{z_{k}}{z_{1}}, q\right)
\end{aligned}
$$

where $k \geq 2$

(4.3.8) and (4.3.11) are easy to prove. (4.3.9) and (4.3.10) could be proved by a direct but lengthy computation. We give here a proof of (4.3.9) using the Cauchy Theorem for contour integrals; (4.3.10) can be proved similarly. We know that $P_{1}\left(\frac{z_{1}}{w}, q\right)$ and $P_{1}\left(\frac{z_{1} q}{w}, q\right)$ converge on the domains $\left\{(z, w)|| q|<| z_{1}|<| w \mid<1\right\}$ and $\left\{(z, w)|| q|<| w|<| z_{1} \mid<1\right\}$ respectively and by (3.11) and (3.9) the limits of $P_{1}\left(\frac{z_{1}}{w}, q\right)$ and $P_{1}\left(\frac{z_{1} q}{w}, q\right)-2 \pi i$ have the same meromorphic continuation on

$$
\left\{\left(z_{1}, w, q\right)|| q|<| z_{1}|<1,| q|<| w \mid<1\right\} .
$$

We denote this meromorphic function by $P\left(z_{1} / w, q\right)$. By (3.11) it is clear that $P\left(z_{1} / w, q\right)$ has a pole at $z_{1}=w$, The term

$$
\operatorname{Res}_{w}\left(\iota_{w, z_{1}}\left(w-z_{1}\right)^{i} z_{1}^{\operatorname{deg} a-1-i} w^{-\operatorname{deg} a} P_{1}\left(\frac{z_{1}}{w}, q\right)\right)
$$

in (4.3.9) is equal to the contour integral

$$
\int_{C_{1}}\left(w-z_{1}\right)^{i} z_{1}^{\operatorname{deg} a-1-i} w^{-\operatorname{deg} a} P\left(z_{1} / w, q\right) d w
$$

where $C_{1}$ is a contour of the form $|w|=R$ with $\left|z_{1}\right|<R<1$. The term

$$
\operatorname{Res}_{w}\left(\iota_{z_{1}, w}\left(w-z_{1}\right)^{i} z_{1}^{\operatorname{deg} a-1-i} w^{-\operatorname{deg} a}\left(P_{1}\left(\frac{z_{1} q}{w}, q\right)-2 \pi i\right)\right)
$$

in (4.3.9) is equal to

$$
\int_{C_{2}}\left(w-z_{1}\right)^{i} z_{1}^{\operatorname{deg} a-1-i} w^{-\operatorname{deg} a} P\left(z_{1} / w, q\right) d w
$$


for a contour $C_{2}$ of the form $|w|=r$ with $|q|<r<\left|z_{1}\right|$. By the Cauchy theorem for contour integrals, the left hand side of (4.3.9) is

$$
\int_{C} \ln \left(\frac{z_{1}}{w}\right) w^{-1} P\left(z_{1} / w, q\right) d w
$$

for a small contour $C$ surrounding $z_{1}$. (Note that when $w$ and $z_{1}$ are close enough, $\ln \left(\frac{z_{1}}{w}\right)$ is well defined). We make the change of variable $z_{1}=\exp \left(z_{1}^{\prime}\right)$ and $w=$ $\exp \left(w^{\prime}\right)$ in (4.3.13), so we have

$$
\begin{aligned}
& (4.3 .13)=\int_{C^{\prime}}\left(-\wp_{1}\left(z_{1}^{\prime}-w^{\prime}, \tau\right)+G_{2}(\tau)\left(z_{1}^{\prime}-w^{\prime}\right)-\pi i\right)\left(w^{\prime}-z_{1}^{\prime}\right)^{-1} d w^{\prime} \\
& =-\frac{1}{2}
\end{aligned}
$$

where $C^{\prime}$ is a contour of $w$ surrounding $z_{1}$. This proves (4.3.9). (4.3.10) can be proved similarly.

Combining Proposition 4.3.2 and 4.3.3, we obtain a recurrent formula:

\section{Proposition 4.3.4.}

$$
\begin{aligned}
& F\left((a, w),\left(a_{1}, z_{1}\right),\left(a_{2}, z_{2}\right), \ldots,\left(a_{n}, z_{n}\right), q\right) \\
= & F\left(\left(a[-1] a_{1}, z_{1}\right),\left(a_{2}, z_{2}\right), \ldots,\left(a_{n}, z_{n}\right), q\right) \\
+ & \pi i F\left(\left(a[0] a_{1}, z_{1}\right),\left(a_{2}, z_{2}\right), \ldots,\left(a_{n}, z_{n}\right), q\right) \\
+ & \sum_{m \in \mathbf{N}} P_{m+1}\left(\frac{z_{1}}{w}, q\right) F\left(\left(a[m] a_{1}, z_{1}\right),\left(a_{2}, z_{2}\right) \ldots,\left(a_{n}, z_{n}\right), q\right) \\
- & \sum_{k=1}^{\infty} \widetilde{G}_{2 k}(q) F\left(\left(a[2 k-1] a_{1}, z_{1}\right),\left(a_{2}, z_{2}\right), \ldots,\left(a_{n}, z_{n}\right), q\right) \\
+ & \sum_{k=2}^{n} \sum_{m \in \mathbf{N}}\left(P_{m+1}\left(\frac{z_{k}}{w}, q\right)-P_{m+1}\left(\frac{z_{k}}{z_{1}}, q\right)\right) \\
& \quad \cdot F\left(\left(a_{1}, z_{1}\right), \ldots,\left(a[m] a_{k}, z_{k}\right), \ldots,\left(a_{n}, z_{n}\right), q\right) .
\end{aligned}
$$

Note that the right hand side of (4.3.12) is in fact a finite sum, since $a[m] b=0$ for $m$ sufficiently large.

Next we derive a vanishing condition for the 1-point trace. Recall the notation $o(a)$ (Definition 2.1.1), $o(a)=a(\operatorname{deg} a-1)$ for homogeneous $a$, and we extend the definition by linearity. We rewrite Proposition 4.3 .3 in the case $n=1$ as follows:

Proposition 4.3.5. For every $a, b \in V$

$$
\left.\operatorname{tr}\right|_{M} o(a) o(b) q^{L_{0}}=\left.\operatorname{tr}\right|_{M} o(a[-1] b) q^{L_{0}}-\left.\sum_{k=1}^{\infty} \widetilde{G}_{2 k}(q) \operatorname{tr}\right|_{M} o(a[2 k-1] b) q^{L_{0}} .
$$


Proposition 4.3.6. For every $a, b \in V$, we have

$$
\begin{gathered}
\left.\operatorname{tr}\right|_{M} o(a[0] b)=0, \\
\left.t r\right|_{M} o(a[-2] b) q^{L_{0}}+\left.\sum_{k=2}^{\infty}(2 k-1) \widetilde{G}_{2 k}(q) \operatorname{tr}\right|_{M} o(a[2 k-2] b) q^{L_{0}}=0 .
\end{gathered}
$$

Proof. (4.3.14) is a special case of (4.3.3). Since $o(L[-1] a)=(2 \pi i)^{2} o\left(L_{-1} a+L_{0} a\right)=$ 0 , we have

$$
\left.\operatorname{tr}\right|_{M} O(L[-1] a) o(b) q^{L_{0}}=0 .
$$

Applying (4.3.13) to $\left.\operatorname{tr}\right|_{M} o(L[-1] a) o(b) q^{L_{0}}$, and using the facts that $(L[-1] a)[n]=$ $-n a[n-1]$ and $\left.t r\right|_{M} o(a[0] b) q^{L_{0}}=0$, we get (4.3.15).

Remark. Recalling the Laurent series expansion of the Weierstrass $\wp$-function $\wp_{2}(z, \tau)$, we may write $(4.3 .15)$ as

$$
\left.\operatorname{tr}\right|_{M} o\left(\operatorname{Res}_{z}\left(Y[a, z] \wp_{2}(z, \tau) b\right)\right) q^{L_{0}}=0 .
$$

By using the relation $o\left(L[-1]^{k-1} a\right)=0$ and (4.3.13), we can prove that

$$
\left.\operatorname{tr}\right|_{M} O\left(\operatorname{Res}_{z}\left(Y[a, z] \wp_{k}(z, \tau) b\right)\right) q^{L_{0}}=0
$$

for $k \geq 2$. Note that functions 1 and $\wp_{k}(z, \tau),(k \geq 2)$ form a basis of the space of the meromorphic functions on the torus with possible poles at 0 . The operators $\operatorname{Res}_{z}(Y[a, z] f(z))(f(z)$ is a meromorphic functions on the torus with possible poles at 0$)$ are the global vertex operators on the 1-pointed torus as defined in $[\mathrm{Z}]$.

4.4. Convergence of the $q$-trace. The purpose of this subsection is to prove that the formal power series (4.3.2) converges to some analytic function in the domain

$$
\left\{\left(z_{1}, z_{2}, \ldots, z_{n}, q\right)|1>| z_{1}|>\cdots>| z_{n}|>| q \mid\right\}
$$

under a finiteness condition on the vertex operator algebra $V$, and that the limit can be continued to a meromorphic function in $\left\{\left(z_{1}, \ldots, z_{n}, q\right)\left|z_{i} \neq 0,\right| q \mid<1\right\}$.

Finiteness condition $C$. Let $\{V, Y(, z), 1, \omega\}$ be a vertex operator algebra, and let $C_{2}(V)$ be the linear span of elements of type $a(-2) b,\{V, Y(, z), 1, \omega\}$ is said to satisfy the finiteness condition $C$ if the quotient space $V / C_{2}(V)$ is finite dimensional and every element of $V$ is a sum of elements of the form $L_{-i_{1}} \cdots L_{-i_{k}} a$ for $a$ primary, and moreover every homogeneous space $\operatorname{dim} V_{n}$ is finite dimensional.

The quotient space $V / C_{2}(V)$ has an algebraic structure as a commutative Possion algebra. Define $\{a, b\}=a(0) b$ and $a \cdot b=a(-1) b$; then it follows from the Jacobi identity that $\left\{a, C_{2}(V)\right\} \subset C_{2}(V), a \cdot C_{2}(V) \subset C_{2}(V),\{a, b\}+\{b, a\} \in C_{2}(V)$, $\{\{a, b\}, d\}+\{\{b, d\}, a\}+\{\{d, a\}, b\} \in C_{2}(V), a \cdot b-b \cdot a \in C_{2},(a \cdot b) \cdot d-a \cdot(b \cdot d) \in C_{2}$ and $\{a, b d\}-b\{a, d\}-\{a, b\} d=0$.

By using the algebraic structure of $V / C_{2}(V)$ defined as above, it is easy to show that all the rational vertex operator algebras mentioned in Section 1.3 satisfy the 
finiteness condition $C$. We conjecture that the rationality implies the finiteness condition $C$.

By Proposition 4.3.4, we see that, in order to prove $F\left(\left(a_{1}, z_{1}\right), \ldots,\left(a_{n}, z_{n}\right), q\right)$ converges on the domain (4.4.1), it is sufficient to prove that the one-point function $F((a, z), q)=\left.\operatorname{tr}\right|_{M} o(a) q^{L_{0}}$ converges on $\{q|| q \mid<1\}$. Considering $V\left[\widetilde{G}_{4}(q), \widetilde{G}_{6}(q)\right]$ $\subset V[[q]]$, it is a module over the $\operatorname{ring} \mathbf{C}\left[\widetilde{G}_{4}(q), \widetilde{G}_{6}(q)\right]$. Motivated by Proposition 4.3.6, we define $O_{q}(V)$ to be the submodule of $V\left[\widetilde{G}_{4}(q), \widetilde{G}_{6}(q)\right]$ generated by elements of the type

$$
a[-2] b+\sum_{k=2}^{\infty}(2 k-1) \widetilde{G}_{2 k}(q) a[2 k-2] b .
$$

We begin with the study of the quotient module $V\left[\widetilde{G}_{4}(q), \widetilde{G}_{6}(q)\right] / O_{q}(V)$. For convenience we denote by $R$ the $\operatorname{ring} \mathbf{C}\left[\widetilde{G}_{4}(q), \widetilde{G}_{6}(q)\right]$. It is clear that $R$ is Noetherian.

Lemma 4.4.1. If $V$ satisfies the finiteness condition $C$, then

$$
V\left[\widetilde{G}_{4}(q), \widetilde{G}_{6}(q)\right] / O_{q}(V)
$$

is a finitely-generated R-module.

Proof. Note that there are two vertex operator algebra structures involved here: $\{V, Y(, z), 1, \omega\}$ and $\{V, Y[, z], 1, \widetilde{\omega}\}$. Since $a[-2]=a(-2)+\sum_{i \geq-1} k_{i} a(i)$ for some constants $k_{i}$ (they depend on $\operatorname{deg} a$ ), and $a(-2)=a[-2]+\sum_{i>-1} l_{i} a[i]$ for some constants $l_{i}$, we can prove that $V / C_{2}(V)$ finite dimensional implies that $V / C_{2}[V]$ is finite dimensional (where $C_{2}[V]$ denotes the linear span of elements $a[-2] b$ ). Since $V / C_{2}[V]$ is finite dimensional and $C_{2}[V]$ is a direct sum of homogeneous subspaces with respect to the degree [deg], there exists $N$ such that elements $a$ satisfying [deg] $a>N$ are in $C_{2}[V]$. Let $A$ be the $R$-submodule of $V\left[\widetilde{G}_{4}(q), \widetilde{G}_{6}(q)\right]$ generated by $\bigoplus_{n<N} V[n]$. We claim that every element $a$ in $V$ is congruent to some element in $A \operatorname{Mod} O_{q}(V)$.

We prove the claim by using induction on $[\operatorname{deg}] a$. If $[\operatorname{deg}] a \leq N$, there is nothing to prove. Suppose the claim is true for every $a$ with [deg] $a<K$; then for [deg] $a=$ $K>N$ we have $a \in C_{2}[V]$, i.e.,

$$
a=\sum_{i=1}^{s} b_{i}[-2] c_{i} .
$$

We may assume that $[\operatorname{deg}]\left(b_{i}[-2] c_{i}\right)=[\operatorname{deg}] a=K$ for each $i$; we have

$$
b_{i}[-2] c_{i} \equiv-\sum_{k=2}^{\infty}(2 k-1) \widetilde{G}_{2 k}(q) b_{i}[2 k-2] c_{i} \quad \operatorname{Mod} O_{q}(V)
$$

Since $[\operatorname{deg}]\left(b_{i}[2 k-2] c_{i}\right) \leq K$, by the induction assumption, we have

$$
\sum_{k=2}^{\infty}(2 k-1) \widetilde{G}_{2 k}(q) b_{i}[2 k-2] c_{i} \equiv x \text { Mod } O_{q}[V]
$$

for some $x \in A$. By (4.4.3), (4.4.4) and (4.4.5), we thus prove that the claim is true for $a$ with $[\operatorname{deg}] a=K$. 
Lemma 4.4.2. For every element $a \in V$, there exist $s \in \mathbf{N}$ and $g_{i}(q) \in R(i=$ $0,1, \ldots, s-1)$ such that

$$
L[-2]^{s} a+\sum_{i=0}^{s-1} g_{i}(q) L[-2]^{i} a \in O_{q}(V)
$$

Proof. By Lemma 4.4.1, $V\left[\widetilde{G}_{4}(q), \widetilde{G}_{6}(q)\right] / O_{q}(V)$ is a finitely-generated $R$-module, so it is a Noetherian $R$-module. Thus the submodule generated by $L[-2]^{i} a(i \in \mathbf{N})$ is finitely generated. So the claim in the lemma is true.

Lemma 4.4.3. For every $a \in V$

$$
\left.\operatorname{tr}\right|_{M} o(\widetilde{\omega}) o(a) q^{L_{0}}=\left.(2 \pi i)^{2}\left(q \frac{d}{d q}-\frac{c}{24}\right) \operatorname{tr}\right|_{M} o(a) q^{L_{0}} .
$$

Proof. It is clear that $\left.t r\right|_{M} o(\omega) o(a) q^{L_{0}}=\left.\left(q \frac{d}{d q}\right) \operatorname{tr}\right|_{M} o(a) q^{L_{0}}$. Then the lemma follows from relations $\widetilde{\omega}=(2 \pi i)^{2}\left(\omega-\frac{c}{24}\right), o(\widetilde{\omega})=(2 \pi i)^{2}\left(L_{0}-\frac{c}{24}\right)$.

Lemma 4.4.4. If $a \in V$ is a highest weight vector for the Virasoro algebra, and $\left\{i_{1}, \ldots, i_{n}\right\}$ is a sequence of positive integers, then there exist elements $g_{i}(q) \in$ $\mathbf{C}\left[\widetilde{G}_{2}(q), \widetilde{G}_{4}(q), \widetilde{G}_{6}(q)\right](i=0,1, \ldots, n)$ such that

$$
\left.\operatorname{tr}\right|_{M} o\left(L\left[-i_{1}\right] \ldots L\left[-i_{n}\right] a\right) q^{L_{0}}=\left.\sum_{i=0}^{n} g_{i}(q)\left(q \frac{d}{d q}\right)^{i} \operatorname{tr}\right|_{M} o(a) q^{L_{0}} .
$$

Proof. Use induction on $n$. The case $n=0$ is trivial. Assuming the case $n$ is true, we want to prove that the case $n+1$ is also true.

If $i_{1}=1, o\left(L\left[-i_{1}\right] L\left[-i_{2}\right] \ldots L\left[-i_{n+1}\right] a\right)$ is 0 by (4.3.14). So we may assume $i_{1}>1$. Note that

$$
L\left[-i_{1}\right]=\widetilde{\omega}\left[-i_{1}+1\right]=\frac{1}{\left(i_{1}-1\right) !}\left(L[-1]^{i_{1}-2} \widetilde{\omega}\right)[-1],
$$

and denote $b=\frac{1}{\left(i_{1}-1\right) !} L[-1]^{i_{1}-2} \widetilde{\omega} ;$ then

$$
\begin{aligned}
& \left.\operatorname{tr}\right|_{M} o\left(L\left[-i_{1}\right] \ldots L\left[-i_{n+1}\right] a\right) q^{L_{0}} \\
& =\left.\operatorname{tr}\right|_{M} o\left(b[-1] L\left[-i_{2}\right] \ldots L\left[-i_{n+1}\right] a\right) q^{L_{0}} \\
& =\left.\operatorname{tr}\right|_{M} o(b) o\left(L\left[-i_{2}\right] \ldots L\left[-i_{n+1}\right] a\right) q^{L_{0}} \\
& \quad+\left.\sum_{k=1}^{\infty} \widetilde{G}_{2 k}(q) \operatorname{tr}\right|_{M} o\left(b[2 k-1] L\left[-i_{2}\right] \ldots L\left[-i_{n+1}\right] a\right) q^{L_{0}} .
\end{aligned}
$$

If $i_{1}=2$, then

$$
\begin{aligned}
& \left.\operatorname{tr}\right|_{M} o(b) o\left(L\left[-i_{2}\right] \ldots L\left[-i_{n+1}\right] a\right) q^{L_{0}} \\
& \quad=\left.(2 \pi i)^{2}\left(q \frac{d}{d q}-\frac{c}{24}\right) \operatorname{tr}\right|_{M} o\left(L\left[-i_{2}\right] \ldots L\left[-i_{n+1}\right] a\right) q^{L_{0}} .
\end{aligned}
$$


If $i_{1}>2$ then $o(b)=0$, and therefore

$$
\left.\operatorname{tr}\right|_{M} o(b) o\left(L\left[-i_{2}\right] \ldots L\left[-i_{n+1}\right] a\right) q^{L_{0}}=0 .
$$

Note that $b[2 k-1](k \geq 1)$ is a linear combination of operators $L[j]$ with $j \geq 0$, so $b[2 k-1] L\left[-i_{2}\right] \ldots L\left[-i_{n+1}\right] a$ can be expressed as a linear combination of elements of the type $L\left[-k_{1}\right] \ldots L\left[-k_{n}\right] a$. Then we can apply the induction assumption to

$$
b[2 k-1] L\left[-i_{2}\right] \ldots L\left[-i_{n+1}\right] a,
$$

and combining this with (4.4.9) and (4.4.10) completes the proof.

Remark 4.4.1. Consider

$$
\left.\operatorname{tr}\right|_{M} O\left(L[-2]^{m} a\right) q^{L_{0}}
$$

for $a$ as in Lemma 4.4.4. By Lemma 4.4.6

$$
\left.\operatorname{tr}\right|_{M} o\left(L[-2]^{n} a\right) q^{L_{0}}=\left.\sum_{i=0}^{n} g_{i}(q)\left(q \frac{d}{d q}\right)^{i} \operatorname{tr}\right|_{M} o(a) q^{L_{0}},
$$

and we see from the proof of Lemma 4.4.6 that the leading coefficient $g_{n}(q)$ is $(2 \pi i)^{2 m}$.

Theorem 4.4.1. Assume $V$ satisfies finiteness condition $C$. Then $F_{M}((a, z), q)=$ $\left.t r\right|_{M} O(a) q^{L_{0}}$ converges absolutely and uniformly in every closed subset of the domain $\{q|| q \mid<1\}$ for every $a \in V$, and the limit function can be written as $q^{h} f(q)$, where $f(q)$ is some analytic function in $\{q|| q \mid<1\}$.

Proof. By Lemma 4.4.4, we may assume that $a$ is a highest weight vector for the Virasoro algebra. Using Lemma 4.4.2, we have

$$
L[-2]^{s} a+\sum_{i=0}^{s-1} g_{i}(q) L[-2]^{i} a \in O_{q}(V)
$$

for some $s \in \mathbf{N}$ and $g_{i}(q) \in R(i=0,1, \ldots, s-1)$. By the definition of $O_{q}(V)$ and Proposition 4.3.6, (4.4.12) implies

$$
\left.\operatorname{tr}\right|_{M} o\left(L[-2]^{s} a+\sum_{i=0}^{s-1} g_{i}(q) L[-2]^{i} a\right) q^{L_{0}}=0 .
$$

Applying Lemma 4.4.4 and Remark 4.4.1 to the terms $\left.t r\right|_{M} O\left(L[-2]^{i} a\right) q^{L_{0}}$ in (4.4.13), we obtain a differential equation for the formal series $\left.\operatorname{tr}\right|_{M} o(a) q^{L_{0}}$ :

$$
\left.\left(q \frac{d}{d q}\right)^{s} \operatorname{tr}\right|_{M} o(a) q^{L_{0}}+\left.\sum_{i=0}^{s-1} h_{i}(q)\left(q \frac{d}{d q}\right)^{i} t r\right|_{M} o(a) q^{L_{0}}=0,
$$

where $h_{i}(q) \in \mathbf{C}\left[\widetilde{G}_{2}(q), \widetilde{G}_{4}(q), \widetilde{G}_{6}(q)\right]$. Since $h_{i}(q)$ converges absolutely and uniformly on every closed subset of $\{q|| q \mid<1\}$, and the equation (4.4.14) is regular, it follows that $\left.\operatorname{tr}\right|_{M} O(a) q^{L_{0}}$ converges uniformly on every closed subset of $\{q|| q \mid<1\}$. The remainder of the theorem is clear.

Since $P_{m}(x, q)$ converges on the domain $\{(x, q)|1>| x|>| q \mid\}$ to a holomorphic function, the limit can continued to a meromorphic function on $\{(x, q)|x \neq 0| q \mid,<$ $1\}$, and $\widetilde{G}_{m}(q)$ converges on the domain $\{q|| q \mid<1\}$, Using these facts together with Proposition 4.3.4 and Theorem 4.4.1, we obtain 
Theorem 4.4.2. Assume $V$ satisfies the finiteness condition $C$. Then for every $\left\{a_{1}, \ldots, a_{n}\right\} \subset V, F\left(\left(a_{1}, z_{1}\right), \ldots,\left(a_{n}, z_{n}\right), q\right)$ converges absolutely to an analytic function on the domain

$$
\left\{\left(z_{1}, z_{2}, \ldots, z_{n}, q\right)|1>| z_{1}|>\cdots>| z_{n}|>| q \mid\right\},
$$

and the limit can continued to a meromorphic function in the domain

$$
\left\{\left(z_{1}, z_{2}, \ldots, z_{n}, q\right)\left|z_{i} \neq 0, z_{i} \neq z_{j} q^{k}(k \in \mathbf{Z}),\right| q \mid<1\right\} .
$$

Let $\widetilde{F}_{M}\left(\left(a_{1}, z_{1}\right), \ldots,\left(a_{n}, z_{n}\right), q\right)$ be the meromorphic continuation of the limit of $F_{M}\left(\left(a_{1}, z_{1}\right), \ldots,\left(a_{n}, z_{n}\right), q\right)$, so $\widetilde{F}_{M}$ above is a meromorphic function of the variables $z_{i}, q$ on the domain (4.4.15). We make a change of variables to define $S_{M}$ :

$$
\begin{aligned}
& S_{M}\left(\left(a_{1}, z_{1}\right), \ldots,\left(a_{n}, z_{n}\right), \tau\right) \\
& =\widetilde{F}_{M}\left(\left(a_{1}, e^{2 \pi i z_{1}}\right), \ldots,\left(a_{n}, e^{2 \pi i z_{n}}\right), q\right) q^{-\frac{c}{24}} .
\end{aligned}
$$

Proposition 4.4.1.

$$
\sum_{k=1}^{n} S_{M}\left(\left(a_{1}, z_{1}\right), \ldots,\left(a[0] a_{k}, z_{k}\right), \ldots,\left(a_{n}, z_{n}\right), \tau\right)=0 .
$$

This is a reformulation of Proposition 4.3.1.

Recall (Section 3) that the limits of $P_{1}\left(q_{z}, q\right), P_{2}\left(q_{z}, q\right)$ and $P_{k}\left(q_{z}, q\right)(k \geq 3)$ $\left(q_{z}=e^{2 \pi z}, q=e^{2 \pi \tau}\right)$ are

$$
\begin{aligned}
& -\wp_{1}(z, \tau)+G_{2}(\tau) z-\pi i, \\
& \wp_{2}(z, \tau)+G_{2}(\tau), \\
& (-1)^{k} \wp_{k}(z, \tau), \quad k \geq 3,
\end{aligned}
$$

respectively. Using these formulas and Proposition 4.4.1, we can rewrite Proposition 4.3.4 as follows:

Proposition 4.4.2. We have the recurrent formula

$$
\begin{aligned}
& S_{M}\left((a, w),\left(a_{1}, z_{1}\right),\left(a_{2}, z_{2}\right), \ldots,\left(a_{n}, z_{n}\right), \tau\right) \\
& =S_{M}\left(\left(a[-1] a_{1}, z_{1}\right),\left(a_{2}, z_{2}\right), \ldots,\left(a_{n}, z_{n}\right), \tau\right) \\
& -\sum_{k=2}^{\infty} G_{2 k}(\tau) S_{M}\left(\left(a[2 k-1] a_{1}, z_{1}\right),\left(a_{2}, z_{2}\right), \ldots,\left(a_{n}, z_{n}\right), \tau\right) \\
& +\sum_{m \in \mathbf{N}} \wp_{m+1}\left(w-z_{1}, \tau\right) S_{M}\left(\left(a[m] a_{1}, z_{1}\right), \ldots,\left(a_{n}, z_{n}\right), \tau\right) \\
& +\sum_{k=2}^{n} \sum_{m \in \mathbf{N}}\left(\wp_{m+1}\left(w-z_{k}, \tau\right)-\wp_{m+1}\left(z_{1}-z_{k}\right)\right) \\
& \quad \cdot S_{M}\left(\left(a_{1}, z_{1}\right), \ldots,\left(a[m] a_{k}, z_{k}\right), \ldots,\left(a_{n}, z_{n}\right), \tau\right) .
\end{aligned}
$$

$S_{M}$ defines a map for every $n \geq 1$ :

$$
S_{M, n}: V^{n} \rightarrow \mathcal{F}_{n}, \quad\left(a_{1}, \ldots, a_{n}\right) \mapsto S_{M}\left(\left(a_{1}, z_{1}\right), \ldots,\left(a_{n}, z_{n}\right), \tau\right)
$$

(the fact that $S_{M}\left(\left(a_{1}, z_{1}\right), \ldots,\left(a_{n}, z_{n}\right), \tau\right)$ is in $\mathcal{F}_{n}$ will be proved later).

We now arrive at the main theorem of this section. 
Theorem 4.4.3. If the vertex operator algebra $\{V, Y(, z), 1, \omega\}$ satisfies the finiteness condition $C . M=\sum_{n=0}^{\infty} M_{n}$ is a representation of $V$ such that $\operatorname{dim} M_{n}<\infty$ and $L_{0}$ acts on $M_{n}$ as $n+h$ for some constant $h$, then every $q$-trace

$$
\left.\operatorname{tr}\right|_{M} Y\left(a_{1}, z_{1}\right) \cdots Y\left(a_{n}, z_{n}\right) q^{L_{0}}
$$

converges absolutely on every closed subset of the domain $1>\left|z_{1}\right|>\ldots\left|z_{n}\right|>|q|>$ 0 , and the limit has a meromorphic continuation on the domain $1>\left|z_{i}\right|>|q|>0$ $(i=1, \ldots, n)$. Let

$$
\left.\operatorname{Tr}\right|_{M} Y\left(a_{1}, z_{1}\right) \cdots Y\left(a_{n}, z_{n}\right) q^{L_{0}}
$$

denote the meromorphic continuation, and set

$$
\begin{aligned}
& S_{M}\left(\left(a_{1}, z_{1}\right), \ldots,\left(a_{n}, z_{n}\right), \tau\right) \\
& =\left.e^{2 \pi i z_{1} \operatorname{deg} a_{1}} \cdots e^{2 \pi i z_{n} \operatorname{deg} a_{n}} \operatorname{Tr}\right|_{M} Y\left(a_{1}, e^{2 \pi i z_{1}}\right) \cdots Y\left(a_{n}, e^{2 \pi i z_{n}}\right) e^{2 \pi i \tau L_{0}} e^{-\frac{2 \pi i \tau}{24}}
\end{aligned}
$$

let $\{V, Y[, z], 1, \widetilde{\omega}\}$ be the transformed vertex operator algebra as defined in Section 4.2. Then $\left\{S_{M, n}\right\}$ satisfies the genus-one property for $\{V, Y[, z], \widetilde{\omega}, 1\}$. Therefore $M$ gives a vector in the conformal block on the torus.

Proof. We need to prove that

$$
S_{M}\left(\left(a_{1}, z_{1}\right), \ldots,\left(a_{n}, z_{n}\right), \tau\right) \in \mathcal{F}_{n} .
$$

We first prove (1), (2) and (3) in Definition 4.1.1. (1) and (3) are trivial. To prove (2), recall Proposition 4.3.2; we have

$$
\begin{aligned}
& F\left(\left(a_{1}, z_{1}\right), \ldots,\left(a_{j}, z_{j}\right),(a, w),\left(a_{j+1}, z_{j+1}\right), \ldots,\left(a_{n}, z_{n}\right), q\right) \\
& =\left.z_{1}^{\operatorname{deg} a_{1}} \ldots z_{n}^{\operatorname{deg} a_{n}} \operatorname{tr}\right|_{M} a(\operatorname{deg} a-1) Y\left(a_{1}, z_{1}\right) Y\left(a_{2}, z_{2}\right) \ldots Y\left(a_{n}, z_{n}\right) q^{L_{0}} \\
& +\sum_{k=1}^{j} \sum_{m \in \mathbf{N}}\left(P_{m+1}\left(\frac{z_{k} q}{w}, q\right)-2 \pi i \delta_{m, 0}\right) F\left(\left(a_{1}, z_{1}\right), \ldots,\left(a[m] a_{k}, z_{k}\right), \ldots,\left(a_{n}, z_{n}\right), q\right) \\
& +\sum_{k=j+1}^{n} \sum_{m \in \mathbf{N}} P_{m+1}\left(\frac{z_{k}}{w}, q\right) F\left(\left(a_{1}, z_{1}\right), \ldots,\left(a[m] a_{k}, z_{k}\right), \ldots,\left(a_{n}, z_{n}\right), q\right) .
\end{aligned}
$$

Since analytic continuations of $P_{m+1}(x, q)$ and $P_{m+1}(x q, q)-2 \pi i \delta_{m, 0}$ are identical (see Section 3), therefore

$$
\begin{aligned}
& S_{M}\left(\left(a_{1}, z_{1}\right), \ldots,\left(a_{j}, z_{j}\right),(a, w),\left(a_{j+1}, z_{j+1}\right), \ldots,\left(a_{n}, z_{n}\right), \tau\right) \\
& =S_{M}\left((a, w),\left(a_{1}, z_{1}\right), \ldots,\left(a_{n}, z_{n}\right), \tau\right) .
\end{aligned}
$$

This proves (2).

Now we can prove (4.4.18). By (4.4.19), we only need to prove that

$$
S_{M}\left((a, w),\left(a_{1}, z_{1}\right), \ldots,\left(a_{n}, z_{n}\right), \tau\right)
$$


is doubly periodic for $w$ with periods $s \tau+t(s, t \in \mathbf{Z})$. Using Proposition 4.4.2, we see that the terms with coefficients $1, G_{2 k}(\tau)$ and $\wp_{m}(m \geq 2)$ in (4.4.17) are doubly periodic for $w$, so we only need to prove that

$$
\sum_{k=1}^{n} \wp_{1}\left(w-z_{i}, \tau\right) S_{M}\left(\left(a_{1}, z_{1}\right), \ldots,\left(a[0] a_{k}, z_{k}\right), \ldots,\left(a_{n}, z_{n}\right), \tau\right)
$$

is doubly periodic for $w$, and this is proved using (3.9) and Proposition 4.4.1. This completes the proof of (4.4.18).

To prove (4) in Definition 4.1.1, we note that $L[-1]=2 \pi i\left(L_{-1}+L_{0}\right)$. We have

$$
\begin{aligned}
S_{M} & \left(\left(L[-1] a_{1}, z_{1}\right), \ldots,\left(a_{n}, z_{n}\right), \tau\right) \\
= & 2 \pi i S_{M}\left(\left(L_{-1} a_{1}, z_{1}\right), \ldots,\left(a_{n}, z_{n}\right), \tau\right) \\
& +2 \pi i S_{M}\left(\left(L_{0} a_{1}, z_{1}\right), \ldots,\left(a_{n}, z_{n}\right), \tau\right) \\
= & 2 \pi i e^{2 \pi i z_{1}\left(\operatorname{deg} a_{1}+1\right)} \ldots e^{2 \pi i z_{n} \operatorname{deg} a_{n}} \operatorname{tr} Y\left(L_{-1} a_{1}, e^{2 \pi i z_{1}}\right) \ldots Y\left(a_{n}, e^{2 \pi i z_{n}}\right) q^{L_{0}-\frac{c}{24}} \\
& +2 \pi i e^{2 \pi i z_{1} \operatorname{deg} a_{1}} \ldots e^{2 \pi i z_{n} \operatorname{deg} a_{n}} \operatorname{tr} Y\left(L_{0} a_{1}, e^{2 \pi i z_{1}}\right) \ldots Y\left(a_{n}, e^{2 \pi i z_{n}}\right) q^{L_{0}-\frac{c}{24}}
\end{aligned}
$$

Plugging

$$
Y\left(L_{-1} a, e^{2 \pi i z}\right)=\frac{1}{2 \pi i} e^{-2 \pi i z} \frac{d}{d z} Y\left(a, e^{2 \pi i z}\right)
$$

into the last term, we prove (4).

To prove (5) in Definition 4.1.1, i.e., to prove that

$$
\begin{aligned}
& \int_{C} S_{M}\left((a, w),\left(a_{1}, z_{1}\right), \ldots,\left(a_{n}, z_{n}\right), \tau\right)\left(w-z_{1}\right)^{k} d w \\
& =S_{M}\left(\left(a[k] a_{1}, z_{1}\right),\left(a_{2}, z_{2}\right), \ldots,\left(a_{n}, z_{n}\right), \tau\right)
\end{aligned}
$$

for $C$ a contour surrounding $z_{1}$, we note that (4.4.21) is true for $k \geq-1$ by Proposition 4.4.2. For $k \geq 1$, using (4), we have

$$
\begin{aligned}
& \int_{C} S_{M}\left((a, w),\left(a_{1}, z_{1}\right), \ldots,\left(a_{n}, z_{n}\right), \tau\right)\left(w-z_{1}\right)^{-k-1} d w \\
& =\int_{C} S_{M}\left((a, w),\left(a_{1}, z_{1}\right), \ldots,\left(a_{n}, z_{n}\right), \tau\right) \frac{(-1)^{k}}{k !}\left(\frac{d}{d w}\right)^{k}\left(w-z_{1}\right)^{-1} d w \\
& =\frac{1}{k !} \int_{C}\left(\frac{d}{d w}\right)^{k} S_{M}\left((a, w),\left(a_{1}, z_{1}\right), \ldots,\left(a_{n}, z_{n}\right), \tau\right)\left(w-z_{1}\right)^{-1} d w \\
& =\frac{1}{k !} \int_{C} S_{M}\left(\left(L[-1]^{k} a, w\right),\left(a_{1}, z_{1}\right), \ldots,\left(a_{n}, z_{n}\right), \tau\right)\left(w-z_{1}\right)^{-1} d w \\
& =\frac{1}{k !} S_{M}\left(\left(\left(L[-1]^{k} a\right)[-1] a_{1}, z_{1}\right), \ldots,\left(a_{n}, z_{n}\right), \tau\right) \\
& =S_{M}\left(\left(\left(a[-k-1] a_{1}, z_{1}\right), \ldots,\left(a_{n}, z_{n}\right), \tau\right),\right.
\end{aligned}
$$

as desired.

Finally, (6) in Definition 4.1.4 is a corollary of Proposition 4.3.2. 


\section{Modular InVARIANCE OF RATIONAL VERTEX OPERATOR ALGEBRAS}

In Section 4 we proved that for a vertex operator algebra $\{V, Y(, z), 1, \omega\}$ satisfying the finiteness condition $C$, a $V$-module $M=\bigoplus_{n=0}^{\infty} M_{n}$ with $\operatorname{dim}\left(M_{n}\right)<\infty$ and $\left.L_{0}\right|_{M_{n}}=h+n$ gives a vector in the conformal block on the torus for $\{V, Y[, z], 1, \widetilde{\omega}\}$ by taking the $q$-trace of products of vertex operators on $M$. The purpose of this section is to prove that if $\{V, Y(, z), 1, \omega\}$ is rational and satisfies the finiteness condition $C$, then its irreducible modules $M^{1}, \ldots, M^{n}$ gives a basis of the conformal block on the torus for $\{V, Y[, z], 1, \widetilde{\omega}\}$. This result together with the action of $S L_{2}(\mathbf{Z})$ on the conformal block (which is given below) implies that the linear space of the characters of $M^{1}, \ldots, M^{n}$ is invariant under $S L_{2}(\mathbf{Z})$.

5.1. $S L_{2}(\mathbf{Z})$ action on the conformal blocks on the torus. Let $\{V, Y(, z), 1, \omega\}$ be a vertex operator algebra, $\{V, Y[, z], 1, \widetilde{\omega}, 1\}$ the transformed vertex operator algebra defined in Section 4.2. We denote the weight of $a \in V$ given by $L[0]$ by [deg] $a$ as before.

Theorem 5.1.1. If

$$
S_{n}: V^{n} \rightarrow \mathcal{F}_{n}, \quad\left(a_{1}, \ldots, a_{n}\right) \mapsto S\left(\left(a_{1} z_{1}\right), \ldots,\left(a_{n}, z_{n}\right), \tau\right)
$$

$(n=1,2, \ldots)$ satisfies the genus one property for $\{V, Y[, z], 1, \widetilde{\omega}\}$, and if for

$$
\sigma=\left(\begin{array}{ll}
a & b \\
c & d
\end{array}\right) \in S L_{2}(\mathbf{Z})
$$

we define $\sigma(S)_{n}: V^{n} \rightarrow \mathcal{F}_{n}$ by

$$
\begin{aligned}
& \sigma(S)\left(\left(a_{1}, z_{1}\right), \ldots,\left(a_{n}, z_{n}\right), \tau\right) \\
& =(c \tau+d)^{-\sum_{i=1}^{n}[\operatorname{deg}] a_{i}} S\left(\left(a_{1}, \frac{z_{1}}{c \tau+d}\right), \ldots,\left(a_{n}, \frac{z_{n}}{c \tau+d}\right), \frac{a \tau+b}{c \tau+d}\right),
\end{aligned}
$$

then $\left\{\sigma(S)_{n}\right\}(n=1,2, \ldots)$ also satisfies the genus one property for $\{V, Y[, z], 1, \widetilde{\omega}\}$. Thus $\mathrm{SL}_{2}(\mathbf{Z})$ acts on the conformal block on the torus.

Proof. It is easy to check that for fixed $\tau$,

$$
\sigma(S)\left(\left(a_{1}, z_{1}\right), \ldots,\left(a_{n}, z_{n}\right), \tau\right)
$$

has periods $a \tau+b$ and $c \tau+d$ for each variable $z_{i}$; since $\operatorname{det}(\sigma)=1,1$ and $\tau$ are also periods. This proves $(5.1 .2) \in \mathcal{F}_{n}$.

It is obvious that $\sigma(S)$ satisfies (1), (2), (3) in Definition 4.1.1. To prove (4) in Definition 4.1.1, we have

$$
\begin{aligned}
& \sigma(S)\left(\left(L[-1] a_{1}, z_{1}\right), \ldots,\left(a_{n}, z_{n}\right), \tau\right) \\
& =(c \tau+d)^{-1-\sum_{i=1}^{n}[\operatorname{deg}] a_{i}} S\left(\left(L[-1] a_{1}, \frac{z_{1}}{c \tau+d}\right), \ldots,\left(a_{n}, \frac{z_{n}}{c \tau+d}\right), \frac{a \tau+b}{c \tau+d}\right) \\
& =(c \tau+d)^{-1-\sum_{i=1}^{n}[\operatorname{deg}] a_{i}} \frac{d}{d\left(z_{1} / c \tau+d\right)} S\left(\left(a_{1}, \frac{z_{1}}{c \tau+d}\right), \ldots,\left(a_{n}, \frac{z_{n}}{c \tau+d}\right), \frac{a \tau+b}{c \tau+d}\right) \\
& =(c \tau+d)^{-\sum_{i=1}^{n}[\operatorname{deg}] a_{i}} \frac{d}{d z_{1}} S\left(\left(a_{1}, \frac{z_{1}}{c \tau+d}\right), \ldots,\left(a_{n}, \frac{z_{n}}{c \tau+d}\right), \frac{a \tau+b}{c \tau+d}\right) \\
& =\frac{d}{d z_{1}} \sigma(S)\left(\left(a_{1}, z_{1}\right), \ldots,\left(a_{n}, z_{n}\right), \tau\right) .
\end{aligned}
$$


This proves (4). (5) can be proved similarly. (6) is proved using the relations

$$
\begin{aligned}
& G_{2}\left(\frac{a \tau+b}{c \tau+d}\right)=(c \tau+d)^{2} G_{2}(\tau)-2 \pi i c(c \tau+d) \\
& \wp_{n}\left(\frac{z}{c \tau+d}, \frac{a \tau+b}{c \tau+d}\right)=(c \tau+d)^{n} \wp_{n}(z, \tau) .
\end{aligned}
$$

5.2. Finiteness of the conformal block. In this subsection we prove some properties of correlation functions on the torus, and we show that the finiteness condition $C$ implies the conformal block on the torus is finite dimensional. These results will be used to prove the modular invariance of the characters of the irreducible representations of rational vertex operator algebras.

Recall that $S((a, z), \tau)$ is an elliptic function of $z$ with no poles (see Section 4.1), so it is independent of $z$. We will write $S(a, \tau)$ for $S((a, z), \tau)$.

Proposition 5.2.1. If $S_{n}: V^{n} \rightarrow \mathcal{F}_{n}(n=1,2, \ldots)$ satisfies the genus one property for $\{V, Y[, z], \widetilde{\omega}, 1\}$, then the following statements (1)-(5) hold.

(1). For every $a, a_{1}, \ldots, a_{n} \in V$

$$
\sum_{k=1}^{n} S\left(\left(a_{1}, z_{1}\right), \ldots,\left(a[0] a_{k}, z_{k}\right), \ldots,\left(a_{n}, z_{n}\right), \tau\right)=0
$$

(2). We have the recurrent formula

$$
\begin{aligned}
& S\left((a, w),\left(a_{1}, z_{1}\right),\left(a_{2}, z_{2}\right), \ldots,\left(a_{n}, z_{n}\right), \tau\right) \\
& =S\left(\left(a[-1] a_{1}, z_{1}\right),\left(a_{2}, z_{2}\right), \ldots,\left(a_{n}, z_{n}\right), \tau\right) \\
& -\sum_{k=2}^{\infty} G_{2 k}(\tau) S\left(\left(a[2 k-1] a_{1}, z_{1}\right),\left(a_{2}, z_{2}\right), \ldots,\left(a_{n}, z_{n}\right), \tau\right) \\
& +\sum_{m \in \mathbf{N}} \wp_{m+1}\left(w-z_{1}, \tau\right) S\left(\left(a[m] a_{1}, z_{1}\right), \ldots,\left(a_{n}, z_{n}\right), \tau\right) \\
& +\sum_{k=2}^{n} \sum_{m \in \mathbf{N}}\left(\wp_{m+1}\left(w-z_{k}, \tau\right)-\wp_{m+1}\left(z_{1}-z_{k}, \tau\right)\right) \\
& \quad \cdot S\left(\left(a_{1}, z_{1}\right), \ldots,\left(a[m] a_{k}, z_{k}\right), \ldots,\left(a_{n}, z_{n}\right), \tau\right) .
\end{aligned}
$$

(3). $S(a[0] b, \tau)=0$ for every $a, b \in V$.

(4). $S(x, \tau)=0$ for

$$
x=\operatorname{Res}_{z}\left(Y[a, z] b \wp_{2}(z, \tau)\right)=a[-2] b+\sum_{k=2}^{\infty}(2 k-1) G_{2 k}(\tau) a[2 k-2] b .
$$

(5). If $a$ is a highest weight vector for the Virasoro algebra and $n_{i}(i=1, \ldots k)$ are positive integers, then there exists a linear differential operator $L\left(\frac{d}{d \tau}\right)$ with order at most $k$ and coefficients polynomials of $\left(\frac{d}{d \tau}\right)^{m} G_{2 n}(\tau)$ such that

$$
S\left(L\left[-n_{1}\right] \ldots L\left[-n_{k}\right] a, \tau\right)=L\left(\frac{d}{d \tau}\right) S(a, \tau) .
$$


Moreover, $L\left(\frac{d}{d \tau}\right)$ depends only on the data $\left(\operatorname{deg} a, n_{1}, n_{2}, \ldots, n_{k}, c\right)$, where $c$ is the rank of $V$. Moreover if $n_{1}=n_{2}=\cdots=n_{k}=2$, then $L\left(\frac{d}{d \tau}\right)$ has order $k$ and the leading coefficient is $(2 \pi i)^{k}$.

We remark that for $\left\{S_{n}\right\}$ given by a $V$-module $M$, the properties (1)-(5) in Proposition 5.2.1 are proved in Section 4 and used to prove the genus one property. (1) is in Proposition 4.4.1, (2) is Proposition 4.4.2, (3) and (4) are essentially Proposition 4.3.6, and (5) is Lemma 4.4.4. Here we proceed in the opposite direction to prove that (1)-(5) are consequences of the genus one property.

Proof of Proposition 5.2.1. (1). Considering $S\left((a, w),\left(a_{1}, z_{1}\right), \ldots,\left(a_{n}, z_{n}\right), \tau\right)$, fix $z_{1}, \ldots, z_{n}, \tau . S$ is an elliptic function of $w$; its possible poles are at $z_{1}, \ldots, z_{n}$ (up to periods $s \tau+t)$. Since the sum of residues is 0 , we have

$$
\sum_{i=1}^{n} \int_{C_{i}} S\left((a, w),\left(a_{1}, z_{1}\right), \ldots,\left(a_{n}, z_{n}\right), \tau\right) d w=0
$$

where $C_{i}$ is a contour surrounding $z_{i}$. By property (5) in Definition 4.1.1,

$$
\begin{aligned}
& \int_{C_{i}} S\left((a, w),\left(a_{1}, z_{1}\right), \ldots,\left(a_{n}, z_{n}\right), \tau\right) d w \\
& =S\left(\left(a_{1}, z_{1}\right), \ldots,\left(a[0] a_{i}, z_{i}\right), \ldots,\left(a_{n}, z_{n}\right), \tau\right) .
\end{aligned}
$$

Plugging (5.2.4) into the left hand side of (5.2.3), we prove (1).

$(2)$. Let $A\left(w, z_{1}, \ldots, z_{n}\right)$ be the difference of the two sides of (5.2.2). We view $A\left(w, z_{1}, \ldots, z_{n}\right)$ as an elliptic function of $w$; it has poles at $z_{1}, z_{2}, \ldots, z_{n}$ (up to periods $s \tau+t)$. Using property (5) in Definition 4.1.1, we can prove that

$$
\int_{C_{i}} A\left(w, z_{1}, \ldots, z_{n}\right)\left(w-z_{i}\right)^{k} d w=0
$$

for every $n \geq i \geq 1$ and $k \geq 0$. This means $A\left(w, z_{1}, \ldots, z_{n}\right)$ has no poles for $w$, so $A\left(w, z_{1}, \ldots, z_{n}\right)$ is independent of $w$. So it is sufficient to prove

$$
\int_{C} A\left(w, z_{1}, \ldots, z_{n}\right)\left(w-z_{1}\right)^{-1} d w=0
$$

for a contour $C$ surrounding $z_{1}$, which again follows from a direct computation and property (5) in Definition 4.1.1.

(3). $S(a[0] b, \tau)=0$ is a special case of (1), which we just proved.

(4). Considering $S((a, z),(b, w), \tau)$, we have

$$
\int_{C} S((a, z),(b, w), \tau) \wp_{2}(z-w, \tau) d z=0
$$

for a contour surrounding $w$. Since $\wp_{2}(z-w, \tau)$ has the power series expansion

$$
\frac{1}{(z-w)^{2}}+\sum_{n=1}^{\infty}(2 n+1) G_{2 n+2}(\tau)(z-w)^{2 n}
$$


(see (3.6)), substituting (5.2.6) into (5.2.5), then using property (5) in Definition 4.1.1, we prove (4).

(5). By property (5) in Definition 4.1.1, we have

$$
\begin{aligned}
& S\left(L\left[-n_{1}\right] \ldots L\left[-n_{k}\right] a, \tau\right) \\
& =\int_{C_{1}} \ldots \int_{C_{k}} S\left(\left(\widetilde{\omega}, w_{1}\right), \ldots,\left(\widetilde{\omega}, w_{k}\right),(a, z), \tau\right) \\
& \quad \cdot\left(w_{1}-z\right)^{-n_{1}+1} \ldots\left(w_{k}-z\right)^{-n_{k}+1} d w_{1} \ldots d w_{k}
\end{aligned}
$$

where $C_{i}$ is the contour for $w_{i}, C_{i}$ contains $C_{i+1}$, and $C_{k}$ contains $z$.

Using property (6) in Definition 4.1.1, we see that the right hand side of (5.2.7) is of the form $L\left(\frac{d}{d \tau}\right) S(a, \tau)$, where the differential operator $L\left(\frac{d}{d \tau}\right)$ has the required properties. In the case $n_{1}=n_{2}=\cdots=n_{k}=2$, it is clear that $L\left(\frac{d}{d \tau}\right)$ has order $k$ and the leading coefficient is $(2 \pi i)^{k}$.

Proposition 5.2.2. If $V$ satisfies the finiteness condition $C$ (see Section 4.4), then there exist in $V$ a finite number of highest weight vectors $b_{1}, \ldots, b_{n}$ for the Virasoro algebra, such that for $\left\{S_{n}\right\}(n=1,2, \ldots)$ satisfying the genus-one property for $\{V, Y[, z], 1, \widetilde{\omega}\}$, every $S\left(\left(a_{1}, z_{1}\right), \ldots,\left(a_{k}, z_{k}\right), \tau\right)$ can be written as

$$
S\left(\left(a_{1}, z_{1}\right), \ldots,\left(a_{k}, z_{k}\right), \tau\right)=\sum_{i=1}^{n} \sum_{j=0}^{N} f_{i, j}\left(\frac{d}{d \tau}\right)^{j} S\left(b_{i}, \tau\right)
$$

for some $N$ and $f_{i, j}$ a polynomial of $\wp_{m}\left(z_{s}-z_{t}, \tau\right)(m=1,2, \tau ; s \neq t), G_{m}(\tau)(m=$ $2,4, \ldots)$ and their derivatives with respect to $\tau, N$; and $f_{i, j}$ depends only on $a_{1}, \ldots, a_{k}, b_{1}, \ldots, b_{n}$ (but not on $S$ ).

Proof. Considering $V\left[G_{4}(\tau), G_{6}(\tau)\right]$, which a module over the $\operatorname{ring} \mathbf{C}\left[G_{4}(\tau), G_{6}(\tau)\right]$, we define a submodule $O_{\tau}(V)$ of $V\left[G_{4}(\tau), G_{6}(\tau)\right]$ which is generated by elements of the type

$$
a[-2] b+\sum_{k=2}^{\infty}(2 k-1) G_{2 k} a[2 k-2] b .
$$

Since $C_{2}(V)$ has finite codimension in $V$ (finiteness condition $C$ ), by Lemma 4.4.1 the quotient module $V\left[G_{4}(\tau), G_{6}(\tau)\right] / O_{\tau}(V)$ is finitely generated. Therefore there exist finite elements $c_{1}, \ldots, c_{l}$ in $V$ such that for every $x \in V\left[G_{4}(\tau), G_{6}(\tau)\right]$ we have

$$
x \equiv \sum_{i=1}^{l} f_{i}(\tau) c_{i} \quad \operatorname{Mod} O_{\tau}(V)
$$

for some $f_{i}(\tau) \in \mathbf{C}\left[G_{4}(\tau), G_{6}(\tau)\right]$. There exist a finite number of highest weight vectors $b_{1}, \ldots, b_{n}$ such that each $c_{i}$ is a linear combination of $L\left[-j_{1}\right] \ldots L\left[-j_{s}\right] b_{j}$. So $x \in V\left[G_{4}(\tau), G_{6}(\tau)\right]$, and we have

$$
x \equiv \sum_{i=1}^{m} g_{i}(\tau) L\left[-i_{1}\right] \ldots L\left[-i_{s}\right] b_{i} \quad \operatorname{Mod} O_{\tau}(V)
$$

for $g_{i}$ a polynomial of $G_{2 i}$ 's. 
We claim that $b_{1}, \ldots, b_{n}$ satisfy the required properties in our proposition, because $S\left(\left(a_{1}, z_{1}\right), \ldots,\left(a_{n}, z_{n}\right)\right)$ can be written in terms of one point functions $S(x, \tau)$ by the recurrent formula (5.2.2), and each one point function $S(x, \tau)$ can be written as a sum of $S\left(L\left[-i_{1}\right] \ldots L\left[-i_{s}\right] b_{i}, \tau\right)$ by (5.2.8), and by (5) in Proposition 5.2.1, $S\left(L\left[-i_{1}\right] \ldots L\left[-i_{s}\right] b_{i}, \tau\right)$ is $L\left(\frac{d}{d \tau}\right) S\left(b_{i}, \tau\right)$ for some differential operator $L\left(\frac{d}{d \tau}\right)$.

Proposition 5.2.3. If $V$ satisfies the finiteness condition $C$ (see Section 4.4), and $b$ is a highest weight vector for the Virasoro algebra, then there exists an ordinary linear differential operator $L\left(\frac{d}{d \tau}\right)$ with leading term $\left(2 \pi i \frac{d}{d \tau}\right)^{n}$ for some $n$ and the coefficients for lower terms $\left(\frac{d}{d \tau}\right)^{i}$ being polynomials of $\left(\frac{d}{d \tau}\right)^{i} G_{2 j}(\tau)$ such that for every $\left\{S_{n}\right\}(n=1,2, \ldots)$ satisfying the genus-one property for $\{V, Y[, z], 1, \widetilde{\omega}\}$, $S(b, \tau)$ satisfies the differential equation $L\left(\frac{d}{d \tau}\right) S(b, \tau)=0$.

Proof. The proof is the same as that of Theorem 4.4.1. Since $V$ satisfies the finiteness condition $C, V\left[G_{4}(\tau), G_{6}(\tau)\right] / O_{\tau}(V)$ is finitely generated over $\mathbf{C}\left[G_{4}(\tau), G_{6}(\tau)\right]$, so there exsit

$$
L[-2]^{s} b+\sum_{i=0}^{s-1} g_{i}(\tau) L[-2]^{i} b \in Q_{\tau}(V)
$$

so

$$
S\left(L[-2]^{s} b, \tau\right)+\sum_{i=0}^{s-1} g_{i}(\tau) S\left(L[-2]^{i} b, \tau\right)=0 .
$$

Applying (5) in Proposition 5.2.1 to the left hand side of (5.2.9), we prove the proposition.

Theorem 5.2.1. If $V$ satisfies the finiteness condition $C$, then the conformal block on the torus for $\{V, Y[, z], 1, \widetilde{\omega}\}$ is finite dimensional.

Proof. Let $b_{1}, \ldots, b_{n}$ be as in Proposition 5.2.2; it suffices to prove that each $S\left(b_{i}, \tau\right)$ is in a finite dimensional space. But this is true because $S\left(b_{i}, \tau\right)$ satisfies an ordinary differential equation by Proposition 5.2.3.

5.3. Modular invariance. In this subsection, we prove that if $V$ is a rational vertex operator algebra and satisfies the finiteness condition $C$, then its conformal block on the torus has a basis

$$
\left\{S_{M}\left(\left(a_{1}, z_{1}\right), \ldots,\left(a_{n}, z_{n}\right), \tau\right)\right\}
$$

for $M$ ranging over the set of irreducible representations of $V\left(\left\{S_{M}\right\}\right.$ is defined in (4.4.16)).

A crucial step of our proof involves the study of the top coefficients of the $q$ expansion of one point functions $S(a, \tau)$ (after we prove that a certain $q$-expansion exists). We will prove that the top coefficients come from the top level of a $V$ module; in the proof, we use the associative algebra $A(V)$ constructed in Section 2 .

We first prove some lemmas which will be needed later.

Let $V \otimes \mathcal{F}_{1}$ be the linear space consisting of $\sum_{i=1}^{n} f_{i}(\tau) a_{i}$ for $f_{i}(\tau) \in \mathcal{F}_{1}$. Motivated by Proposition 4.3.5, we define an operation $\underset{\widetilde{\omega}}{\tau} * a$ for $a \in V \otimes \mathcal{F}_{1}$. We first 
define $\underset{\tau}{\widetilde{\omega}} * \underset{\tau}{a}$ for $a \in V$ :

$$
\begin{aligned}
\underset{\tau}{\widetilde{\omega} * a} & =\operatorname{Res}_{z}\left(Y[\widetilde{\omega}, z] b\left(\wp_{1}(z, \tau)-G_{2}(\tau) z\right)\right) \\
& =L[-2] a-\sum_{k=1}^{\infty} G_{2 k}(\tau) L[2 k-2] a .
\end{aligned}
$$

In general, for $f(\tau) a(a \in V)$, we define

$$
\widetilde{\omega} * f(\tau) a=f(\tau) \widetilde{\omega} \underset{\tau}{\tau} a+2 \pi i \frac{d}{d \tau} f(\tau) a .
$$

This operation enjoys the following property, as suggested by Proposition 4.3 .5 and Lemma 4.4.3.

Lemma 5.3.1. If $\{S\}$ satisfies the genus-one property with respect to the vertex operator algebra $\{V, Y[, z], 1, \widetilde{\omega}\}$, then for every $a \in V \otimes \mathcal{F}_{1}$ we have

$$
S(\widetilde{\omega} * a, \tau)=2 \pi i \frac{d}{d \tau} S(a, \tau) .
$$

Proof. We only need to prove (5.3.2) for $a \in V$. If $a$ is a highest weight vector for the Virasoro algebra, then

$$
S((\widetilde{\omega}, w),(a, z), \tau)=2 \pi i \frac{d}{d \tau} S(a, \tau)+\operatorname{deg} a\left(\wp_{2}(w-z, \tau)+G_{2}(\tau)\right) S(a, \tau) .
$$

Multiplying both sides by $\wp_{1}(w-z, \tau)-G_{2}(\tau)(w-z)$, then taking the contour integral for $w$ along a contour surrounding $z$, the left hand side is $\underset{\tau}{S(\widetilde{\omega} * a, \tau)}$ by $(5)$ in Definition 4.4.1; and using the identity

$$
\int_{C}\left(\wp_{2}(w-z, \tau)+G_{2}(\tau)\right)\left(\wp_{1}(w-z, \tau)-G_{2}(\tau)(w-z)\right) d w=0,
$$

the right hand side is $2 \pi i \frac{d}{d \tau} S(a, \tau)$. This proves (5.3.2) for $a$ a highest weight vector of the Virasoro algebra.

If $a=L\left[-i_{1}\right] \cdots L\left[-i_{n}\right] b$, where $b$ is a highest weight vector for the Virasoro algebra, then we have

$$
\begin{aligned}
& S(\widetilde{\omega} * a, \tau) \\
& =\int_{C} \int_{C_{1}} \ldots \int_{C_{n}} S\left((\widetilde{\omega}, w),\left(\widetilde{\omega}, w_{1}\right), \ldots,\left(\widetilde{\omega}, w_{n}\right),(b, z), \tau\right) \\
& \cdot\left(w_{1}-z\right)^{-i_{1}+1} \ldots\left(w_{n}-z\right)^{-i_{n}+1}\left(\wp_{1}(w-z, \tau)-G_{2}(\tau)(w-z)\right) d w d w_{1} \ldots d w_{n},
\end{aligned}
$$

where the contour for $w_{i}$ contains the contour for $w_{i+1}$, and the contour for $w_{n}$ contains $z ; C$ is a contour for $w$ containing all the other contours. By the Cauchy Theorem for contour integrals, we have

$$
\begin{aligned}
& S(\widetilde{\omega} * a, \tau) \\
& =\sum_{k=0}^{n} \int_{C_{1}} \ldots \int_{C_{n}} \int_{C^{k}} S\left((\widetilde{\omega}, w),\left(\widetilde{\omega}, w_{1}\right), \ldots,\left(\widetilde{\omega}, w_{n}\right),(b, z), \tau\right) \\
& \cdot\left(w_{1}-z\right)^{-i_{1}+1} \ldots\left(w_{n}-z\right)^{-i_{n}+1}\left(\wp_{1}(w-z, \tau)-G_{2}(\tau)(w-z)\right) d w d w_{1} \ldots d w_{n},
\end{aligned}
$$


where we take the contour integral for $w$ first along small contours $C^{k}$ surrounding $w_{k}$ and $C^{0}$ surrounding $z$. Using (6) in Definition 4.1.1, we can prove that

$$
\begin{aligned}
& \sum_{k=0}^{n} \int_{C^{k}} S\left((\widetilde{\omega}, w),\left(\widetilde{\omega}, w_{1}\right), \ldots,\left(\widetilde{\omega}, w_{n}\right),(b, z), \tau\right) \\
& \cdot\left(\wp_{1}(w-z, \tau)-G_{2}(\tau)(w-z)\right) d w \\
& =2 \pi i \frac{d}{d \tau} S\left(\left(\widetilde{\omega}, w_{1}\right), \ldots,\left(\widetilde{\omega}, w_{n}\right),(b, z), \tau\right) .
\end{aligned}
$$

Substituting this identity into (5.3.3), we prove (5.3.2).

Since we will study the top coefficients of $q$-expansions of a one point function $S(a, \tau)$, we need to know the implications of (4) in Proposition 5.2.1 and Lemma 5.3.1 for the top coefficients if its $q$-expansion exists. For this purpose, we view

$$
a[-1] b-\sum_{k=1}^{\infty} G_{2 k}(\tau) a[2 k-1] b
$$

and

$$
a[-2] b+\sum_{k=2}^{\infty}(2 k-1) G_{2 k}(\tau) a[2 k-2] b
$$

as elements in $V[[q]]$ by taking the $q$-expansions of $G_{2 k}(\tau)$. We need to find their constant terms.

Lemma 5.3.2. If we view (5.3.4) and (5.3.5) as elements in $V[[q]]$, then their constant terms are $a * b-\frac{1}{2} a[0] b$ and

$$
\frac{\pi i}{6} a[0] b+2 \pi i \operatorname{Res}_{z}\left(Y(a, z) \frac{(1+z)^{\operatorname{deg} a}}{z^{2}} b\right),
$$

respectively, where $a * b$ is defined in Section 2.1 .

Proof. Using (3.7), we have

$$
a[-1] b-\sum_{k=1}^{\infty} G_{2 k}(\tau) a[2 k-1] b=\operatorname{Res}_{z}\left(Y[a, z]\left(\wp_{1}(z, \tau)-G_{2}(\tau) z\right)\right) b ;
$$

using (3.7), the constant of the $q$-expansion of (5.3.6) is

$$
\begin{aligned}
& \pi i \operatorname{Res}_{z}\left(Y[a, z] \frac{e^{2 \pi i z}+1}{e^{2 \pi i z}-1}\right) b \\
& =\pi i \operatorname{Res}_{z}\left(Y\left(a, e^{2 \pi i z}-1\right) e^{2 \pi i z \operatorname{deg} a} \frac{e^{2 \pi i z}+1}{e^{2 \pi i z}-1}\right) b \\
& =\operatorname{Res}_{w}\left(Y(a, w) \frac{(1+w)^{\operatorname{deg} a}}{2 w} \frac{(w+2)}{(1+w)}\right) b \\
& =a * b-\frac{1}{2} a[0] b .
\end{aligned}
$$

Using (3.8) and a similar computation, the constant term of (5.3.5) is

$$
\frac{\pi i}{6} a[0] b+2 \pi i \operatorname{Res}_{z}\left(Y(a, z) \frac{(1+z)^{\operatorname{deg} a}}{z^{2}} b\right) .
$$


Lemma 5.3.3. Let $A$ be a semi-simple associative algebra over $\mathbf{C}, \omega$ an element in the center of $A$, and $F$ a linear functional of $A$ satisfying

(1) $F(a b)=F(b a)$ for every $a, b \in A$,

(2) $F\left((\omega-h)^{s} a\right)=0$ for every $a \in A$, where $h$ is constant and $s$ is a fixed positive integer.

Then there exist finite dimensional A-modules $M_{1}, \ldots, M_{n}$ on which $\omega$ acts as a scalar $h$ and constants $k_{1}, \ldots, k_{n}$ such that

$$
F(a)=\left.\sum_{i=1}^{n} k_{i} t r\right|_{M_{i}} a
$$

for every $a \in A$.

Proof. The semi-simplicity of $A$ and the first condition imply $F$ is a linear combination of traces for $A$-modules. The second condition implies that only those $A$-modules on which $\omega$ acts as a scalar $h$ appear.

Theorem 5.3.1. If $\{V, Y(, z), 1, \omega\}$ is a rational vertex operator algebra and satisfies the finiteness condition $C$, and $M_{1}, M_{2}, \ldots, M_{m}$ are its complete list of irreducible modules, then the conformal block on the torus for $\{V, Y[, z], 1, \widetilde{\omega}\}$ has a basis

$$
\left\{S_{M_{i}}\left(\left(a_{1}, z_{1}\right), \ldots,\left(a_{n}, z_{n}\right), \tau\right)\right\} \quad(i=1, \ldots, n) .
$$

Proof. Let $M_{i}(0)$ be the top level of $M_{i}$; it defines a linear function $t r_{i}: V \rightarrow \mathbf{C}$ by $\operatorname{tr}_{i}(a)=\left.t r\right|_{M_{i}(0)} o(a)$. This linear function reduces to $t r_{i}: A(V) \rightarrow \mathbf{C}$ by Theorem 2.1.2. Since $M_{1}(0), \ldots, M_{m}(0)$ are non-isomorphic irreducible modules of the semisimple associative algebra $A(V), t r_{1}, \ldots, t r_{m}$ are linearly independent. It follows that the linear maps $V \rightarrow \mathcal{F}_{1}: a \mapsto S_{M_{i}}(a, \tau)(i=1, \ldots, m)$ are linearly independent. So the vectors in the conformal block given by $M_{1}, \ldots, M_{m}$ are linearly independent. It remains to prove that they span the conformal block.

To show that the system $\left\{S\left(\left(a_{1}, z_{1}\right), \ldots\left(a_{k}, z_{k}\right), \tau\right)\right\}$ satisfies the genus-one property for $\{V, Y[, z], 1, \widetilde{\omega}\}$, we need to prove that there are constants $c_{1}, \ldots, c_{m}$ such that

$$
S\left(\left(a_{1}, z_{1}\right), \ldots,\left(a_{k}, z_{k}\right), \tau\right)=\sum_{i=1}^{m} c_{i} S_{M_{i}}\left(\left(a_{1}, z_{1}\right), \ldots,\left(a_{k}, z_{k}\right), \tau\right) .
$$

Let $b_{1}, \ldots, b_{n}$ be as in Proposition 5.2.2; then by Proposition 5.2.2, every one point function $S(a, \tau)$ can be expressed as $\sum f_{i j}(\tau)\left(\frac{d}{d \tau}\right)^{j} S\left(b_{i}, \tau\right)$ where $f_{i j}(\tau)$ has a $q$-expansion of the form $f_{i j}(\tau)=\sum_{l=0}^{\infty} c_{l} q^{l}\left(q=e^{2 \pi i \tau}\right)$. And by Proposition 5.2.3, $S\left(b_{i}, \tau\right)$ satisfies an ordinary differential equation

$$
\left(\frac{d}{d \tau}\right)^{s} S\left(b_{i}, \tau\right)+\sum_{k=0}^{s-1} f_{k}(\tau)\left(\frac{d}{d \tau}\right)^{k} S\left(b_{i}, \tau\right)=0,
$$

where each $f_{k}(\tau)$ has a $q$-expansion of the form $f(\tau)=\sum_{l=0}^{\infty} c_{l} q^{l}$. Note that $\frac{d}{d \tau}=\frac{1}{2 \pi i} q \frac{d}{d q}$, so we may write the differential equation for $S\left(b_{i}, \tau\right)$ in the form

$$
\sum_{i=1}^{s} g_{i}(q)\left(q \frac{d}{d q}\right)^{i} S(b, \tau)=0
$$


where the $g_{i}(q)$ are analytic functions on $\{q|| q \mid<1\}$. By classical results in the theory of ordinary differential equations (see e.g. [In]), the general solutions of (5.3.7) have expressions

$$
\sum_{i=0}^{s}(\ln q)^{i} f_{i}(q)
$$

and the $f_{i}(q)$ can be expanded as power series

$$
\sum_{i=1}^{M} q^{r_{i}} \sum_{j \in \mathbf{N}} c_{i, j} q^{j}
$$

$\left(r_{i} \in \mathbf{C}, r_{i}-r_{j} \notin \mathbf{Z}\right.$ for $\left.i \neq j\right)$. Consequently, there exists a non-negative integer $N$ such that for every $a \in V$,

$$
S(a, \tau)=\sum_{i=0}^{N} S_{i}(a, \tau)(\ln q)^{i}
$$

and each $S_{i}(a, \tau)$ can be further decomposed as

$$
S_{i}(a, \tau)=\sum_{j=1}^{l_{i}} S_{i j}(a, \tau) q^{r_{i j}},
$$

where $r_{i 1}, \ldots, r_{i l_{i}}$ are complex numbers independent of $a, r_{i j_{1}}-r_{i j_{2}} \notin \mathbf{Z}$ for $j_{1} \neq j_{2}$, and $S_{i j}(a, \tau)$ has $q$-expansion $S_{i j}(a, \tau)=\sum_{k=0}^{\infty} c_{k} q^{k}$.

Our purpose is to prove that $N=0$ (i.e., there is no logarithm function in (5.3.9)) and at the same time to prove that there exsit $c_{1}, \ldots, c_{m}$ such that for every $a \in V$

$$
S(a, \tau)=\sum_{i=1}^{m} c_{i} S_{M_{i}}(a, \tau)
$$

This together with the recurrent formula (5.2.2) implies that

$$
\begin{aligned}
& S\left(\left(a_{1}, z_{1}\right), \ldots,\left(a_{n}, z_{n}\right), \tau\right) \\
& =\sum_{i=1}^{m} c_{i} S_{M_{i}}\left(\left(a_{1}, z_{1}\right), \ldots,\left(a_{n}, z_{n}\right), \tau\right),
\end{aligned}
$$

which will conclude the proof.

We may assume $S_{N}(a, z) \neq 0$ for some $a \in V$. Consider $S_{N}(a, \tau)$. Each $S_{N}(a, \tau)$ has decomposition

$$
S_{N}(a, \tau)=\sum_{j=1}^{l} S_{N j}(a, \tau) .
$$

There exist $r_{1}, \ldots, r_{l}, r_{i}-r_{j} \notin \mathbf{Z}$ for $i \neq j$, such that $S_{N j}(a, \tau)$ has the $q$-expansion:

$$
S_{N j}(a, \tau)=\sum_{i=0}^{\infty} C_{N j, i}(a) q^{i} q^{r_{j}}
$$


By (4) in Proposition 5.2.1, for

$$
x=a[-2] b+\sum_{k=2}^{\infty}(2 k-1) G_{2 k}(\tau) a[2 k-2] b,
$$

$S(x, \tau)=0$, this implies that $S_{N}(x, \tau)=0$, and further that

$$
S_{N j}(x, \tau)=0 .
$$

Similarly, (3) in Proposition 5.2.1 implies that

$$
S_{N j}(a[0] b, \tau)=0 .
$$

Using Lemma 5.3.1, we have

$$
S(\widetilde{\omega} * a, \tau)=2 \pi i \frac{d}{d \tau} S(a, \tau),
$$

and so

$$
S_{N}(\widetilde{\omega} * a, \tau)=2 \pi i \frac{d}{d \tau} S_{N}(a, \tau)
$$

which further implies that

$$
S_{N j}(\widetilde{\omega} \underset{\tau}{*} a, \tau)=2 \pi i \frac{d}{d \tau} S_{N j}(a, \tau) .
$$

The top coefficient $C_{N j, 0}(a)$ defines a linear function $V \rightarrow \mathbf{C}$. We claim that (5.3.11)-(5.3.13) imply the following:

$$
\begin{aligned}
& C_{N j, 0}(a * b)=C_{N j, 0}(b * a), \\
& C_{N j, 0}(a)=0 \quad \text { for } a \in O(V), \\
& C_{N j, 0}\left(\left(\omega-\frac{c}{24}-r_{i}\right) * a\right)=C_{N j, 0}(a)
\end{aligned}
$$

(recall that $a * b, O(V)$ is defined in Section 2, and $c$ is the rank of $V$ ). It is clear that (5.3.11) implies that $C_{N j, 0}(a[0] b)=0$. For $x$ as in (5.3.10), write $x=\sum_{i=0}^{\infty} x_{i} q^{i}$; then (5.3.12) implies that $C_{N j, 0}\left(x_{0}\right)=0$, and by Lemma 5.3.2, the constant term $x_{0}$ is

$$
\frac{\pi i}{6} a[0] b+2 \pi i \operatorname{Res}_{z}\left(Y(a, z) \frac{(1+z)^{\operatorname{deg} a}}{z^{2}}\right) .
$$

This together with $C_{N j, 0}(a[0] b)=0$ proves $(5.3 .15)$. Recall that

$$
a * b-b * a \equiv \frac{1}{2 \pi i} a[0] b \quad \bmod O(V)
$$

(see Section 2 and Section 4.2), so (5.3.14) is true. (5.3.16) can be proved using (5.3.13).

(5.3.14)-(5.3.16) allows us to apply Lemma 5.3 .3 for the associative algebra $A(V)$, so there exist irreducible $A(V)$-modules $W_{1}, \ldots, W_{k}$ and constants $c_{1}, \ldots, c_{k}$ such that $C_{N j, 0}(a)=\left.\sum_{i=1}^{k} c_{i} t r\right|_{W_{i}}(a)$, and $\omega \in A(V)$ acts as a scaler $r_{j}+\frac{c}{24}$ on each 
$W_{i}$; by Theorem 2.2.1, we have corresponding irreducible $V$-modules $\bar{W}_{1}, \ldots, \bar{W}_{k}$. Consider

$$
S_{N j}^{\prime}(a, \tau)=S_{N j}(a, \tau)-\sum_{i=1}^{k} c_{i} S_{\bar{W}_{i}}(a, \tau) .
$$

Note that the top coefficients of the $q$-expansions of $S_{N j}(a, \tau)$ and $\sum_{i=1}^{k} c_{i} S_{\overline{W_{i}}}(a, \tau)$ are the same. Thus there is $r_{j}^{\prime} \in \mathbf{C}$ such that $r_{j}^{\prime}-r_{j}$ is a positive integer and each $S_{N j}^{\prime}$ has the decomposition

$$
S_{N j}^{\prime}=\sum_{i=0}^{\infty} C_{N j, i}^{\prime}(a) q^{i} q^{r_{j}^{\prime}}
$$

This makes it clear that the $S_{N j}^{\prime}(a, \tau)$ 's satisfy (5.3.11) -(5.3.13), so $C_{N j, 0}^{\prime}(0)$ 's satisfy (5.3.14)-(5.3.16), Therefore we may apply the same procedure for $S_{N j}^{\prime}$; we get irreducible $V$-modules $\bar{W}_{1}^{\prime}, \ldots, \bar{W}_{k^{\prime}}^{\prime}$ on whose top levels $L_{0}$ acts on as $r_{j}^{\prime}+\frac{c}{24}$. We form $S_{N j}^{\prime \prime}$; if it is not zero, we continue the same procedure get irreducible $V$-modules $\bar{W}_{1}^{\prime \prime}, \ldots, \bar{W}_{k^{\prime \prime}}^{\prime \prime}$ on whose top levels $L_{0}$ acts on as $r_{j}^{\prime \prime}+\frac{c}{24}$ and $r_{j}^{\prime \prime}-r_{j}^{\prime}$ is a positive integer. Since $V$ has only finite many irreducible modules, this process must stop at a finite stage. So we have proved that there are $V$-modules $W_{1}, \ldots, W_{k}$ and constants $c_{1}, \ldots, c_{k}$ such that for each $a \in V, S_{N j}(a, \tau)=\sum_{i=0}^{k} c_{i} S_{W_{i}}(a, \tau)$.

It remains to prove that $N=0$, i.e., there is no logarithm function $(\ln q)^{i}$ in (5.3.9). For this purpose, we assume the opposite, $N>0$, and prove that this assumption leads to a contradiction. Consider $S_{N-1}(a, \tau)$. There are complex numbers $r_{1}, \ldots, r_{l}, r_{i}-r_{j} \notin \mathbf{Z}$ for $i \neq j$, such that each $S_{N-1}(a, \tau)$ has the decomposition

$$
S_{N}(a, \tau)=\sum_{j=1}^{l} S_{N-1, j}(a, \tau),
$$

where $S_{N-1, j}(a, \tau)$ has $q$-expansion:

$$
S_{N-i, j}(a, \tau)=\sum_{i=0}^{\infty} C_{N-1, j, i}(a) q^{i} q^{r_{j}} .
$$

For $x$ as (5.3.10), $S(x, \tau)=0$. This implies that $S_{N-1}(x, \tau)=0$, and, further,

$$
S_{N-1, j}(x, \tau)=0 .
$$

Similarly, (3) in Proposition 5.2.1 implies that

$$
S_{N-1, j}(a[0] b, \tau)=0 .
$$

Using Lemma 5.3.1, we have

$$
S(\widetilde{\omega} * a, \tau)=2 \pi i \frac{d}{d \tau} S(a, \tau)
$$

this implies that

$$
S_{N-1}(\widetilde{\omega} * a, \tau)=(2 \pi i)^{2} N S_{N}(a, \tau)+2 \pi i \frac{d}{d \tau} S_{N-1}(a, \tau) .
$$


Using this and $S_{N}(\widetilde{\omega} * a, \tau)=2 \pi i \frac{d}{d \tau} S_{N}(a, \tau)$, we can prove that

$$
S_{N-1}(\widetilde{\omega} \underset{\tau}{*} \underset{\tau}{*} a, \tau)=2(2 \pi i) \frac{d}{d \tau} S_{N-1}(\widetilde{\omega} \underset{\tau}{*} a, \tau)-(2 \pi i)^{2}\left(\frac{d}{d \tau}\right)^{2} S_{N-1}(a, \tau),
$$

which in turn implies that

$$
S_{N-1, j}(\widetilde{\omega} \underset{\tau}{*} \underset{\tau}{*} a, \tau)=2(2 \pi i) \frac{d}{d \tau} S_{N-1, j}(\widetilde{\omega} * a, \tau)-(2 \pi i)^{2}\left(\frac{d}{d \tau}\right)^{2} S_{N-1, j}(a, \tau) .
$$

Use $\left(5.3 .11^{\prime}\right)-\left(5.3 .13^{\prime}\right)$, we can prove that the top coefficient $C_{N-1, j, 0}(a)$ has the following property analogous to (5.3.14)-(5.3.16):

$$
\begin{aligned}
& C_{N-1, j, 0}(a * b)=C_{N-1, j, 0}(b * a), \\
& C_{N-1, j, 0}(a)=0 \quad \text { for } a \in O(V), \\
& C_{N-1, j, 0}\left(\left(\omega-\frac{c}{24}-r_{j}\right)^{2} * a\right)=0 .
\end{aligned}
$$

(Note that $\left(5.3 .16^{\prime}\right)$ is slightly more complicated than (5.3.16).) $\left(5.3 .14^{\prime}\right)-\left(5.3 .16^{\prime}\right)$ allow us to apply Lemma 5.3.3 for the associative algebra $A(V)$, so there exist irreducible $A(V)$-modules $W_{1}, \ldots, W_{k}$ and constants $c_{1}, \ldots, c_{k}$ such that $C_{N-1, j, 0}(a)=$ $\left.\sum_{i=1}^{k} c_{i} t r\right|_{W_{i}}(a)$, and $\omega \in A(V)$ acts as a scalar $r_{j}+\frac{c}{24}$ on each $W_{i}$. By Theorem 2.2.1, we have corresponding irreducible $V$-modules $\bar{W}_{1}, \ldots, \bar{W}_{k}$. Consider

$$
S_{N-1, j}^{\prime}(a, \tau)=S_{N-1, j}(a, \tau)-\sum_{i=1}^{k} c_{i} S_{\bar{W}_{i}}(a, \tau) .
$$

Note here that the top coefficients of the $q$-expansions of $S_{N-1, j}(a, \tau)$ and $\sum_{i=1}^{k} c_{i} S_{\bar{W}_{i}}(a, \tau)$ are the same. Thus there is $r_{j}^{\prime} \in \mathbf{C}$ such that $r_{j}^{\prime}-r_{j}$ is a positive integer and each $S_{N-1, j}^{\prime}$ has the decomposition

$$
S_{N-1, j}^{\prime}=\sum_{i=0}^{\infty} C_{N-1, j, i}^{\prime}(a) q^{i} q^{r_{j}^{\prime}} .
$$

This makes it clear that the $S_{N-1, j}^{\prime}(a, \tau)$ 's satisfy formulas $\left(5.3 .11^{\prime}\right)-\left(5.3 .13^{\prime}\right)$, so the $C_{N-1, j, 0}^{\prime}(0)$ 's satisfy $\left(5.3 .14^{\prime}\right)-\left(5.3 .16^{\prime}\right)$, Therefore we may apply the same procedure for $S_{N-1, j}^{\prime}$ to get irreducible $V$-modules $\bar{W}_{1}^{\prime}, \ldots, \bar{W}_{k^{\prime}}^{\prime}$ on whose top levels $L_{0}$ acts as $r_{j}^{\prime}+\frac{c}{24}$. We form $S_{N-1, j}^{\prime \prime}$; if it is not zero, we continue the same procedure to get irreducible $V$-modules $\bar{W}_{1}^{\prime \prime}, \ldots, \bar{W}_{k^{\prime \prime}}^{\prime \prime}$ on whose top levels $L_{0}$ acts as $r_{j}^{\prime \prime}+\frac{c}{24}$ with $r_{j}^{\prime \prime}-$ $r_{j}^{\prime}$ a positive integer. Since $V$ has only finite many irreducible modules, this process must stop at a finite stage. So we have proved that there are $V$-modules $W_{1}, \ldots, W_{k}$ and constants $c_{1}, \ldots, c_{k}$ such that for each $a \in V, S_{N-1, j}(a, \tau)=\sum_{i=0}^{k} c_{i} S_{W_{i}}(a, \tau)$. Therefore there are $V$-modules $W_{1}, \ldots, W_{k}$ and constants $c_{1}, \ldots, c_{k}$ such that for each $a \in V, S_{N-1}(a, \tau)=\sum_{i=0}^{k} c_{i} S_{W_{i}}(a, \tau)$. This implies that

$$
S_{N-1}(\widetilde{\omega} * a, \tau)=2 \pi i \frac{d}{d \tau} S_{N-1}(a, \tau)
$$


Comparing (5.3.17) and (5.3.18), we conclude that

$$
N(2 \pi i)^{2} S_{N}(a, \tau)=0
$$

for every $a \in V$, which contradicts our assumption $N>0$ and $S_{N}(a, \tau) \neq 0$ for some $a$. This completes the proof.

Combining Theorems 4.4.3, 5.1.1 and 5.3.1, we arrive at the following theorem.

Theorem 5.3.2. Let $\{V, Y(, z), 1, \omega\}$ be a rational vertex operator algebra that satisfies the finiteness condition $C$, let $M_{1}, \ldots, M_{m}$ be its complete list of irreducible modules, $c$ be the rank of $V$, and let

$$
S_{M_{i}}\left(\left(a_{1}, z_{1}\right), \ldots,\left(a_{n}, z_{n}\right), \tau\right)
$$

be the meromorphic continuation of the limit

$$
\left.\operatorname{Tr}\right|_{M_{i}} Y\left(e^{2 \pi z_{1} L_{0}} a_{1}, e^{2 \pi z_{1} L_{0}}\right) \cdots Y\left(e^{2 \pi z_{1} L_{0}} a_{n}, e^{2 \pi z_{n} L_{0}}\right) q^{L_{0}-\frac{c}{24}} .
$$

If $a_{1}, \ldots, a_{n}$ are highest weigh vectors for the Virasoro algebra with weights $\operatorname{deg} a_{1}$, $\ldots, \operatorname{deg} a_{n}$, then for every

$$
\alpha=\left(\begin{array}{ll}
a & b \\
f & d
\end{array}\right) \in S l_{2}(\mathbf{Z})
$$

we have

$$
\begin{aligned}
& S_{M_{i}}\left(\left(a_{1}, \frac{z_{1}}{f \tau+d}\right), \ldots,\left(a_{n}, \frac{z_{n}}{f \tau+d}\right), \frac{a \tau+b}{f \tau+d}\right) \\
& =(f \tau+d)^{\sum_{k=1}^{n} \operatorname{deg} a_{k}} \sum_{j=1}^{m} S(\alpha, i, j) S_{M_{j}}\left(\left(a_{1}, z_{1}\right), \ldots,\left(a_{n}, z_{n}\right), \tau\right),
\end{aligned}
$$

where the $S(\alpha, i, j)$ are constants depending only on $\alpha, i, j$. In particular, if $V$ has a unique irreducible representation $M$, and $a$ is a highest weight vector for the Virasoro algebra, then $S_{M}(a, \tau)$ is a modular form of weight $\operatorname{deg}$ a with a certain character.

Note that we can replace the condition " $a_{1}, \ldots, a_{n}$ are highest weight vectors for the Virasoro algebra with weights $\operatorname{deg} a_{1}, \ldots \operatorname{deg} a_{n}$ " by the condition " $a_{1}, \ldots, a_{n}$ are homogeneous for $L[0]$ with weights $\operatorname{deg} a_{1}, \ldots \operatorname{deg} a_{n}$ ". Taking $a_{1}=a_{2}=\cdots=$ $a_{n}=1$ in Theorem 5.3.2, we have

Theorem 5.3.3. If $\{V, Y(, z), 1, \omega\}$ is a rational vertex operator algebra and satisfies the finiteness condition $C$, and $M_{1}, \ldots, M_{m}$ are its complete list of irreducible modules, then the characters $\left.\operatorname{tr}\right|_{M_{i}} q^{L_{0}-\frac{1}{24}}$ converge on the domain $\operatorname{Im}(\tau)>0$ $(q=\exp (2 \pi i \tau))$ to holomorphic functions. Moreover, the linear space spanned by the limits of characters is invariant under the action of $S L_{2}(\mathbf{Z})$.

We mention a few examples to which Theorem 5.3.2 and Theorem 5.3.3 apply. In all the cases, the modular invariance of the characters was known by explicit 
character formulas, but the modular invariance for the general correlation functions of these examples are new in mathematical context.

(1). Let $L$ be a positive definite integral even lattice, ( , ) the pairing. Its associated vertex operator algebra $V_{L}$ is rational and its irreducible representations are in one-to-one correspondence with the cosets in $L^{\prime} / L$, where $L^{\prime}$ is the dual lattice of $L$ [D1]. $V_{L}$ is a unitary representation of the Virasoro algebra; in particular it is a direct sum of highest representations of the Virasoro algebra. It is easy to check that $V_{L} / C_{2}\left(V_{L}\right)$ is finite dimensional, using the Possion algebra structure described in Section 4.4. Thus Theorems 4.4.3, 5.3.2 and 5.3.3 apply to this case. Let $L_{1}, \ldots, L_{m}$ the cosets of $L^{\prime} / L$, and $V_{L_{1}}, \ldots, V_{L_{m}}$ be the corresponding irreducible modules of $V$. The character of $V_{L_{i}}$ is known to be

$$
C H\left(V_{L_{i}}\right)=\sum_{\alpha \in L_{i}} q^{\frac{1}{2}(\alpha, \alpha)}\left(\Pi_{i=1}^{\infty}(1-q)^{i}\right)^{-\operatorname{rank}(L)} q^{-\frac{\operatorname{rank}(\mathrm{L})}{24}}
$$

By Theorem 5.3.2, the linear space $\mathbf{C C H}\left(V_{L_{1}}\right)+\cdots+\mathbf{C} C H\left(V_{L_{n}}\right)$ is invariant under $S L_{2}(\mathbf{Z})$; this fact can also be checked directly using the above character formula. The explicit formula for

$$
\left.\operatorname{tr}\right|_{V_{L_{i}}} Y\left(a_{1}, z_{1}\right) \cdots Y\left(a_{n}, z_{n}\right) q^{L_{0}}
$$

for some special $a_{1}, \ldots, a_{n}$ is computed in [T].

(2). Let $\mathfrak{g}$ be an $n$-dimensional complex simple Lie algebra, $\hat{\mathfrak{g}}$ its associated Kac-Moody affine Lie algebra, and $\left\{\Lambda_{0}, \Lambda_{1}, \ldots, \Lambda_{n}\right\}$ the fundamental weights of $\hat{\mathfrak{g}}$ such that $\left\{\Lambda_{1}, \ldots, \Lambda_{n}\right\}$ are the fundamental weights of $\mathfrak{g}$. For a positive integer $k$, the irreducible highest weight module $L_{k \Lambda_{0}}$ is a rational vertex operator algebra; its irreducible representations are precisely the irreducible integral highest weight $\hat{\mathfrak{g}}$-modules of level $k . L_{k \Lambda_{0}}$ is unitary for the Virasoro algebra, so it is a direct sum of highest weight modules of the Virasoro algebra. It is easy to prove that $L_{k \Lambda_{0}} / C_{2}\left(L_{k \Lambda_{0}}\right)$ is finite dimensional, using the Possion algebra structure described in Section 4.4 and the fact that $r_{\alpha}(-1)$ ( $\alpha$ is a root of $\mathfrak{g}, r_{\alpha}$ is a vector in the root space $\mathfrak{g}_{\alpha}$ ) acts on $L_{k \Lambda}$ nilpotently. The modular invariance of characters in this case is known (see $[\mathrm{F}]$ and $[\mathrm{KP}]$ ) from Weyl-Kac character formula.

(3). Let $L_{c, h}$ be the irrducible highest weight module for the Virosoro algebra with highest weight $(c, h)(c$ corresponds the central charge and $h$ corresponds to $\left.L_{0}\right) . L_{c, 0}$ has a vertex operator algebra structure [FZ]; it is rational if and only if $c=c_{p, q}=1-6(p-q)^{2} / p q$, where $p, q \in\{2,3, \ldots\}$, and $p, q$ are relatively prime [Wa], and its irreducible representations are $L_{c_{p, q}, h_{m, n}}$ for

$$
h_{m, n}=\frac{(n p-m q)^{2}-(p-q)^{2}}{4 p q}
$$

$(0<m<p, 0<n<q)$. In this case, the finiteness condition $C$ can be proved using the fact that there exists a null vector in $M_{c_{p, q}, 0}$ with the leading term as $L_{-2}^{N}$ for some $N$. The character formula for $L_{c, h}$ is computed in [R], based on work in $[\mathrm{FF}]$, The modular invariance of characters is shown in $[\mathrm{Ca}]$ by direct computation.

(4). It is proved in [D1] that the Moonshine module $V^{\natural}$ is rational and $V^{\natural}$ itself is its unique irreducible representation. $V^{\natural}$ is unitary for its Virasoro algebra, so it is a direct sum of irreducible representations. It is found in [DMZ] that there 
are 48 elements $\omega_{1}, \ldots, \omega_{48}$ in $V^{\natural}$ satisfying the following: (1). $\sum_{i=1}^{48} \omega_{i}=\omega$. (2). Each $Y\left(\omega_{i}, z\right)$ gives a Virasoro algebra with central charge $\frac{1}{2}$. (3). The Viraroso algebras for $Y\left(\omega_{i}, z\right)$ and $Y\left(\omega_{j}, z\right)$ are commutative. (4). $V^{\natural}$ can be decomposed as a direct sum of tensor products of unitary highest weight modules for these 48 Viraroso algebras. Using the existence of null vectors in the unitary highest weight representations of Viraroso algebra with central charge $\frac{1}{2}$, it is easy to prove that $V^{\natural}$ satisfies the finiteness condition $C$.

\section{ACKNOWLEDGEMENT}

This paper is based on the author's dissertation submitted to Yale University in 1990. The author wishes to thank his advisor, Prof. Igor Frenkel, for advice. Theorem 5.3.3 was conjectured by him, and he also contributed important ideas to its proof.

\section{REFERENCES}

[Apos] T.M.Apostol, Modular functions and Dirichlet series in number theory, SpringerVerlag, 1976. MR 54:10149

[Bo] R.E. Borcherds, Vertex operator algebras, Kac-Moody algebras and the Monster, Proc. Natl. Acad. Sci. USA. 83 (1986), 3026. MR 87m:17033

[BPZ] A. Belavin, A.M. Polyakov, A.A. Zamolodchikov, Infinite conformal symmetry in two dimensional quantum field theory, Nucl. Phys. B241 (1984), 33. MR 86m:81097

[BS] P.Bouwknegt, K.Schoutens, $W$-symmetry in conformal field theory, Phys. Rep. 223 (1993). MR 94e:81096

[Ca] J.L. Cardy, Operator content of two-dimensional conformal invariant theories, Nucl. Phys. B270 (1986), 186. MR 87k:17017

[Do1] C.Dong, Representation of the moonshine module vertex operator algebra, preprint, 1992.

[Do2] C.Dong, Vertex algebras associated to even lattice, J. of alg. 161 (1993). MR 94j:17023

[DMZ] C.Dong, G.Mason, Y.Zhu, Discrete Series of the Virasoro algebra and the moonshine module, Preprint (1991).

[FFr] B.Feigin, E.Frenkel, Affine Kac-Moody algebras at the critical level and Gelfand-Dikii algebras, Int. J. Mod. Phys. A suppl. 1A (1992). MR 93j:17049

[Fr] I.Frenkel, Orbital theory for affine Lie algebras, Yale dissertation (1980), or Invent. Math. 77 (1984). MR 86d: 17014

[F] E.Frenkel, $W$-algebras and Langlands-Drinfeld correspondence, New Symmetries in Quantum field theory (J. Frohlich, eds.), Plenum Press, New York, 1987.

[FF] B.L. Feigin, D.B. Fuchs, Lect. Notes Math., vol 1060, 1984. MR 86g:17004

[FKRW] E.Frenkel, V.Kac, A.Radul, W.Wang, $W_{1+\infty}$ and $W\left(g l_{N}\right)$ with central charge $N$., Preprint (1994).

[FHL] I.B. Frenkel, Y. Huang, J.Lepowsky, On axiomatic approaches to vertex operator algebras and modules, preprint, 1989; Memoirs American Math. Soc. 104 (1993). MR 94a: 17007

[FLM1] I.B.Frenkel, J.Lepowsky, A.Meurman, A natural representation of the Fischer-Griess Monster with the modular function $J$ as a character, Proc. Nat. Acad. Sci. USA 81 (1984), 3256-3260. MR 85e:20018

[FLM2] I.B.Frenkel, J.Lepowsky, A.Meurman, Vertex Operator Algebras and the Monster, Academic Press, New York, 1988. MR 90h:17026

[FZ] I.B.Frenkel, Y.Zhu, Vertex operator algebras associated to representation of affine and Virasoro algebras, Duke Mathematical Journal 66 (1992), 123. MR 93g:17045

[H] Y.Z.Huang, Geometric interpretation of vertex operator algebras, Proc. Natl. Acad. Sci. USA 88 (1991), 9964. MR 92k:17037

[In] E.L. Ince, Ordinary Differential Equations, Dover Publications, Inc, New York, 1956. MR 6:65f 
[IZ] Itsykson, Zuber, Two-dimensional conformal invariant theories on a torus, Nucl.Phys. B275 (1986). MR 88f: 8111

[K] V.G. Kac, Infinite dimensional Lie algebras and Dedekind's $\eta$-function, Funct. Anal. Appl. 8 (1974), 68-70. MR 51:10410

[KP] V.G. Kac, D.H.Peterson, Infinite-dimensional Lie algebras, theta functions and modular forms, Advances in Math 53 (1984), 124-264. MR 86a:17007

[KW] V.G.Kac, M. Wakimoto, Modular and conformal invariance constraints in representation theory, Adv. Math. 70 (1988). MR 89h:17036

[La] S. Lang, Elliptic Functions, Springer-Verlag, 1987. MR 88c:11028

[Li] B.Lian, On the classification of simple vert ex operator algebras, preprint (1992).

[MS] G. Moore, N. Seiberg, Classical and quantum conformal field theory, Commun. Math. Phys. 123 (1989), 177-254. MR 90e:81216

[R] A. Rocha, in Vertex Operators in Mathematics and Physics, Spring-Verlag, 1983. MR 87b:17011

[TUY] A.Tsuchiya, K.Ueno and Y.Yamada, in Advanced Studies on Pure Math., vol. 19, 1989, pp. 459-566. MR 92a:81191

[T] H.Tsukada, String path integral realization of vertex operator algebras, Memoirs of Amer. Math. Soc. 91,no.444 (1991). MR 91m:17044

[W] N.R.Wallach, Real Reductive Groups I, Academic Press, 1988. MR 89i:22029

[Wa] W.Wang, Rationality of Virasoro vertex operator algebras, Duke Math. J., IMRN 71, No. 1 (1993). MR 94i:17034

[Wi] E. Witten, Quantum field theory, Grassmannians, and algebraic curves, Commun. Math. Phys. 113 (1988), 529-600. MR 88m:81127

[Z] Y.Zhu, Global vertex operators on Riemann surfaces, Commun. Math. Phys. 165 No.3 (1994).

Department of Mathematics, Hong Kong University of Science and Technology, Clear Water Bay, Kowloon, Hong Kong 